AEI-2006-012

\title{
Open-closed TQFTs extend Khovanov homology from links to tangles
}

\author{
Aaron D. Lauda ${ }^{1}$ \\ Department of Pure Mathematics and Mathematical Statistics, \\ University of Cambridge, Cambridge CB3 OWB, United Kingdom \\ Hendryk Pfeiffer ${ }^{2}$ \\ Max Planck Institute for Gravitational Physics, \\ Am Mühlenberg 1, 14476 Potsdam, Germany
}

(June 14, 2006)

\begin{abstract}
We use a special kind of 2-dimensional extended Topological Quantum Field Theories (TQFTs), so-called open-closed TQFTs, in order to extend Khovanov homology from links to arbitrary tangles, not necessarily even. For every plane diagram of an oriented tangle, we construct a chain complex whose homology is invariant under Reidemeister moves. The terms of this chain complex are modules of a suitable algebra $A$ such that there is one action of $A$ or $A^{\text {op }}$ for every boundary point of the tangle. We give examples of such algebras $A$ for which our tangle homology theory reduces to the link homology theories of Khovanov, Lee, and Bar-Natan if it is evaluated for links. As a consequence of the Cardy condition, Khovanov's graded theory can only be extended to tangles if the underlying field has finite characteristic. In all cases in which the algebra $A$ is strongly separable, i.e. for Bar-Natan's theory in any characteristic and for Lee's theory in characteristic other than 2, we also provide the required algebraic operation for the composition of oriented tangles. Just as Khovanov's theory for links can be recovered from Lee's or Bar-Natan's by a suitable spectral sequence, we provide a spectral sequence in order to compute our tangle extension of Khovanov's theory from that of Bar-Natan's or Lee's theory. Thus, we provide a tangle homology theory that is locally computable and still strong enough to recover characteristic $p$ Khovanov homology for links.
\end{abstract}

Mathematics Subject Classification (2000): 57R56, 57M99, 57M25, 57Q45, 18G60.

\section{Introduction}

For every plane diagram of an oriented link $L$, Khovanov's link homology theory [1] yields a chain complex $\llbracket L \rrbracket$ of graded vector spaces whose graded Euler characteristic agrees with the Jones polynomial of the link. This construction can be seen as a categorification of the unnormalized Jones polynomial, replacing a polynomial in one indeterminate $q$ by a chain complex of graded vector spaces. The coefficients of the polynomial arise as the dimensions of the homogeneous components of the graded homology groups of the chain complex in such a way that the degree corresponds to the power of $q$.

If two link diagrams are related by a Reidemeister move, the corresponding chain complexes of graded vector spaces are homotopy equivalent, and so their homology groups are isomorphic as graded vector spaces. This of course implies that their graded Euler characteristics and thereby their unnormalized Jones polynomials agree, but in general the homology groups contain more

\footnotetext{
${ }^{1}$ email: A.Lauda@dpmms.cam.ac.uk

${ }^{2}$ email: pfeiffer@aei.mpg.de
} 
information about the link than does the Jones polynomial. Indeed, Bar-Natan and Wehrli [2-4] have shown that there are knots and links that have the same Jones polynomial, but which can be distinguished by their Khovanov homology.

The construction of Khovanov's chain complex heavily relies on a 2-dimensional Topological Quantum Field Theory (TQFT). The fact that the boundary of a cobordism is a closed manifold, naturally restricts the construction to links rather than arbitrary tangles. The purpose of the present article is to overcome this limitation. In the remainder of the introduction, we give a brief overview of the present article, addressed to the experts. The reader who is familiar with the details of Khovanov's definition [1] and with Bar-Natan's work on the extension from links to tangles [5], should be able to understand the key ideas of our work just from reading the introduction. The reader who is not familiar with all the details, is invited to have a brief look at the introduction in order to get a first impression, and can then work though the material step by step starting from Section 2

\subsection{Overview of Khovanov's link homology theory}

Let $L$ be a plane diagram of an oriented link with $n_{+}$positive crossings ( $\times)$ and $n_{-}$negative ones $\left(\lambda^{\top}\right), n:=n_{+}+n_{-}$. The Kauffman bracket $\langle L\rangle$ from which one can compute the unnormalized Jones polynomial $\widehat{J}(L):=$ $(-1)^{n_{-}} q^{n_{+}-2 n_{-}}\langle L\rangle \in \mathbb{Z}\left[q, q^{-1}\right]$, can be recursively defined ${ }^{3}$ as follows:

$$
\langle\emptyset\rangle:=1 ; \quad\langle\bigcirc L\rangle:=\left(q+q^{-1}\right)\langle L\rangle ; \quad\langle\rtimes\rangle:=\langle\asymp\rangle-q\langle\rangle(\rangle .
$$

For every crossing $(\rtimes)$, each of the two smoothings, the 0 -smoothing $(\asymp)$ and the 1-smoothing ()(), give a contribution to the Kauffman bracket, with a different sign and a different power of $q$. For the link diagram $L$

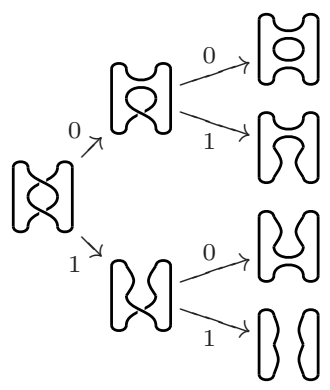
with $n$ crossings, there are $2^{n}$ smoothings, labeled by subsets $\alpha \subseteq \mathbf{n}:=\{1, \ldots, n\}$ where $j \in \alpha$ if the $j$-th crossing is resolved by the 1 -smoothing and $j \notin \alpha$ if it is resolved by the 0 -smoothing. We denote by $D_{\alpha} \subseteq \mathbb{R}^{2}, \alpha \subseteq \mathbf{n}$, the diagrams associated with the $2^{n}$ smoothings of $L$. Each $D_{\alpha}$ is free of crossings and therefore forms a disjoint union of a finite number of circles in $\mathbb{R}^{2}$.

A crucial ingredient for the construction of Khovanov's chain complex is the following collection of cobordisms between these diagrams. For every crossing $j \in \mathbf{n}$ and for all possible smoothings of the other crossings, represented by subsets $\alpha \subseteq \mathbf{n} \backslash\{j\}$, one specifies a 2-dimensional cobordism

$$
S_{(\alpha, j)}: D_{\alpha} \rightarrow D_{\alpha \sqcup\{j\}}
$$

which relates the two possible smoothings of the $j$-th crossing. This cobordism $S_{(\alpha, j)}$ is a cylinder over $D_{\alpha}$ everywhere except for a neighbourhood of the $j$-th $\operatorname{crossing}^{4}$ (メ) where it is a saddle

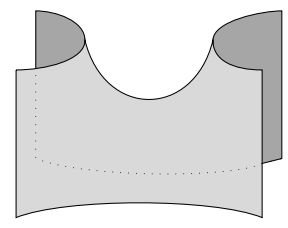

with the 0-smoothing ()() as the source (at the top of the diagram) and the 1-smoothing $(\asymp)$ as the target (at the bottom).

Then one uses a 2-dimensional TQFT, i.e. a symmetric monoidal functor $Z: \mathbf{2 C o b} \rightarrow$ Vect $_{k}$ from the category $\mathbf{2} \mathbf{C o b}$ of 2-dimensional cobordisms to the category Vect $_{k}$ of vector spaces over some fixed field $k$, in order to turn the cobordism into a linear map between vector spaces. The chain groups of Khovanov's chain complex are constructed from the vector spaces $Z\left(D_{\alpha}\right), \alpha \subseteq \mathbf{n}$,

\footnotetext{
${ }^{3}$ We have adopted the conventions of Khovanov [1]. The usual definition of the Jones polynomial is obtained by substituting $-\sqrt{t}$ for $q$ in the normalized polynomial $J(L):=\widehat{J}(L) /\left(q+q^{-1}\right)$.

${ }^{4}$ Notice that we have rotated this picture by 90 degrees.
} 
and the differentials from the linear maps $Z\left(S_{(\alpha, j)}\right), j \in \mathbf{n}, \alpha \subseteq \mathbf{n} \backslash\{j\}$. For more details, see Section 3.3 below, and for the full explanation of how Khovanov's theory is related to the Jones polynomial, we refer to $[1,2]$.

The category of 2-dimensional TQFTs, i.e. the category Symm-Mon(2Cob, Vect $\left.{ }_{k}\right)$ of symmetric monoidal functors $\mathbf{2} \mathbf{C o b} \rightarrow$ Vect $_{k}$ and monoidal natural transformations, is equivalent as a symmetric monoidal category to the category of commutative Frobenius algebras over $k$ [6-8].

The objects of $\mathbf{2} \mathbf{C o b}$ are numbers $k \in \mathbb{N}_{0}$, representing the diffeomorphism types of 1-manifolds that are the disjoint union of $k$ circles. For the morphisms of $\mathbf{2} \mathbf{C o b}$, one has a description in terms of generators and relations. The generators are these cobordisms:

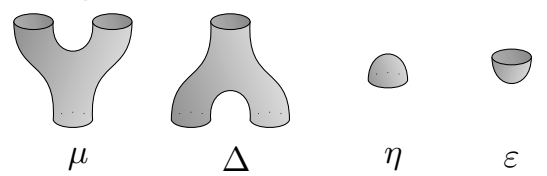

We have drawn the cobordisms with their source at the top and their target at the bottom of the diagram. Composition of morphisms corresponds to putting the building blocks of (1.4) on top of each other. The tensor product of morphisms is the disjoint union of cobordisms. If we denote by $C:=Z(1)$ the vector space associated with a single circle, the functor $Z$ yields linear maps $\mu: C \otimes C \rightarrow C, \Delta: C \rightarrow C \otimes C, \eta: k \rightarrow C$, and $\varepsilon: C \rightarrow k$ for these cobordisms. The relations among the morphisms of $\mathbf{2} \mathbf{C o b}$ are such that $(C, \mu, \eta, \Delta, \varepsilon)$ forms a commutative Frobenius algebra.

Khovanov's original choice of Frobenius algebra, called $A[c]$ in [1], over the commutative ring $R=\mathbb{Z}[c]$, is such that one gets a chain complex of graded modules and a categorification of the Jones polynomial. His TQFT is actually a functor $\mathbf{2} \mathbf{C o b} \rightarrow \operatorname{Mod}_{R}$. In this article, we restrict ourselves to the case $c \equiv 0$ (c.f. $[9,10]$ ) and to algebras over a field $k$. With this restriction, Khovanov's commutative Frobenius algebra forms the special case $h=0, t=0$ of the following definition:

Definition 1.1 (see [11]). Let $k$ be a field and $h, t \in k . C_{h, t}$ denotes the algebra $C_{h, t}=$ $k[x] /\left(x^{2}-h x-t\right)$ equipped with the structure of a commutative Frobenius algebra $\left(C_{h, t}, \mu, \eta, \Delta, \varepsilon\right)$ which is given in the basis $\{1, x\}$ by $\mu(1 \otimes 1)=1, \mu(1 \otimes x)=x, \mu(x \otimes 1)=x, \mu(x \otimes x)=h x+t$, $\eta(1)=1, \Delta(1)=1 \otimes x+x \otimes 1-h \cdot 1 \otimes 1, \Delta(x)=x \otimes x+t \cdot 1 \otimes 1, \varepsilon(1)=0$, and $\varepsilon(x)=1$.

Several results below depend on the characteristic of $k$. While Khovanov originally studied the case $h=0, t=0$, Lee [12] considered $h=0, t=1$, and Bar-Natan [5] studied $h=1, t=0$. Below, we refer to $C_{0,0}$ as Khovanov's, to $C_{0,1}$ as Lee's, and to $C_{1,0}$ as Bar-Natan's Frobenius algebra.

The link homology theory associated with Khovanov's Frobenius algebra is known to categorify the Jones polynomial, a quantum invariant of links. In some cases, the other two link homology theories are related to classical invariants of links: Both Lee's theory in characteristic 0 [12] and Bar-Natan's theory in characteristic 2 [13] categorify combinatorial expressions involving linking numbers.

Bar-Natan [5] has presented sufficient (but not necessary) conditions for TQFTs to yield link homology theories. These can be stated in topological and in algebraic terms as follows:

\section{Definition 1.2.}

1. A 2-dimensional TQFT $Z: \mathbf{2 C o b} \rightarrow$ Vect $_{k}$ is said to satisfy Bar-Natan's conditions if the following three conditions hold:

$$
\begin{aligned}
Z(\theta) & =0, \\
Z\left(f_{0}^{\rho}\right) & =2, \\
Z(\underbrace{\rho}_{0})+Z\left(\begin{array}{l}
\theta \\
\rho
\end{array}\right)-Z\left(g_{0}^{\theta}\right)-Z\left(\begin{array}{l}
\theta \\
0
\end{array}\right) & =0 .
\end{aligned}
$$


2. A commutative Frobenius algebra $(C, \mu, \eta, \Delta, \varepsilon)$ is said to satisfy Bar-Natan's conditions if

$$
\begin{aligned}
\left(\varepsilon_{C} \circ \eta_{C}\right)(1)=0 & (\mathrm{~S}=\text { 'sphere' }), \\
\left(\varepsilon_{C} \circ \mu_{C} \circ \Delta_{C} \circ \eta_{C}\right)(1)=2 & (\mathrm{~T}=\text { 'torus' }), \\
\Delta_{C} \circ \eta_{C} \circ\left(\varepsilon_{C} \otimes \varepsilon_{C}\right)+\left(\eta_{C} \otimes \eta_{C}\right) \circ \varepsilon_{C} \circ \mu_{C} & \\
-\left(\eta_{C} \circ \varepsilon_{C}\right) \otimes \mathrm{id}_{C}-\mathrm{id}_{C} \otimes\left(\eta_{C} \circ \varepsilon_{C}\right)=0 & (4 \mathrm{Tu}=\text { 'four tubes'). }
\end{aligned}
$$

The Frobenius algebra $C_{h, t}$ of Definition 1.1 satisfies Bar-Natan's conditions for all $h, t \in k$. Khovanov [11] has classified the Frobenius algebras that give rise to link homology theories. The algebra $C_{h, t}$ of Definition [1.1] is a specialization of $A_{5}$ of [11] to a field $k$ and to $h, t \in k$. The classification includes examples, for instance Khovanov's $A[c]$ without evaluation at $c=0$, called $A_{2}$ in [11], which do not satisfy all of Bar-Natan's conditions (1.5) to (1.7). In the present article, we restrict ourselves to Frobenius algebras that do satisfy these conditions.

We wish to extend these link homology theories from links to tangles. For tangles, the diagrams $D_{\alpha}$ corresponding to the smoothings would consist not only of circles, but rather of circles and arcs. Khovanov has already remarked in [14] that one would need an extended 2-dimensional TQFT in which the cobordisms are generalized to suitable manifolds with corners.

Even without an extended TQFT, there have been two workarounds. Khovanov [14] considers tangles with an even number of points both for the source and the target of the tangle, so-called even tangles, and presents a definition in which the tangle homology is reduced to his link homology by closing the open ends of the tangles in all possible ways and taking the

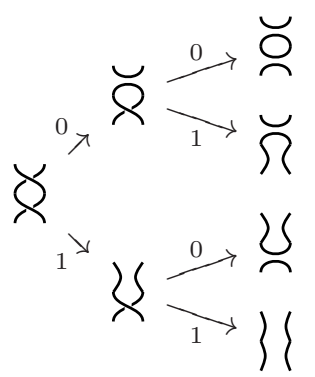
sum over the resulting expressions for the links. He thus obtains a tangle homology theory only for even tangles, but it is still possible to tell which expression this construction categorifies.

Bar-Natan [5] works with formal linear combinations of manifolds with corners. As long as one stays in this topological setting, called the 'picture world', one has good composition laws for tangles, but Bar-Natan still relies on a TQFT and can therefore translate from topology to algebra only for links, i.e. after all open ends have been closed.

The following two questions remain to be answered and form the subject of the present article:

1. How does one define a homology theory for arbitrary oriented tangles, using only algebraic as opposed to topological 'picture world' constructions?

2. Which algebraic operation corresponds to the composition of tangles?

In particular, we wish to understand which algebraic data to assign to a single arc (|) so that one can take a disjoint union of two such arcs $(||)$, then glue on a cap $(\cap)$ and a cup $(\cup)$ in order to obtain a circle in such a way that both operations, disjoint union (tensor product) and gluing (composition) have a correspondence on the algebraic side. If we write $\llbracket T \rrbracket$ for the algebraic data associated with a tangle diagram $T$, this means that:

$$
\llbracket \circ \rrbracket=\llbracket \cup \circ(|\otimes|) \circ \cap \rrbracket=\llbracket \cup \rrbracket \circ(\llbracket|\rrbracket \otimes \llbracket| \rrbracket) \circ \llbracket \cap \rrbracket .
$$

This cannot be done by Khovanov's method [14] since a single arc is not an even tangle, and it cannot be done by Bar-Natan's method [5] since there one can translate from topology to algebra only for links, but not for a single arc which forms a proper tangle.

A further limitation of the existing link homology theories is the following. Recall that the cobordisms $S_{(\alpha, j)}$ of (1.2) are the disjoint union of either $\vartheta$ or $\boldsymbol{S}$ and a number of cylinders over circles. In order to decide which one of the two cobordisms with critical point of index 1 is needed, one has to know the diagrams $D_{\alpha}$ and $D_{\alpha \sqcup\{j\}}$ globally. An extension of Khovanov homology from links to tangles in contrast would provide us with a local description of such a homology theory. This would not only be conceptually more transparent, but also form a valuable property in computations. 


\subsection{Open-closed Topological Quantum Field Theories}

In a previous article [15], we have studied a suitable class of manifolds with corners, called openclosed cobordisms, and classified the corresponding extended TQFTs which we call open-closed TQFTs. They are classified in terms of a custom-made algebraic structure, so-called knowledgeable Frobenius algebras. The category $\mathbf{2} \mathbf{C o b}^{\text {ext }}$ of open-closed cobordisms has a description in terms of generators and relations, too. The objects are sequences $\underline{m}=\left(m_{1}, \ldots, m_{k}\right) \in\{0,1\}^{k}, k \in \mathbb{N}_{0}$, which specify a particular sequence of circles $\left(m_{j}=0\right)$ and $\operatorname{arcs}\left(m_{j}=1\right)$. The generators for the morphisms of $\mathbf{2} \mathbf{C o b}^{\text {ext }}$ are these open-closed cobordisms:

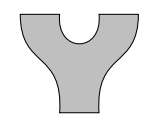

$\mu_{A}$

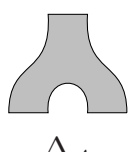

$\Delta_{A}$

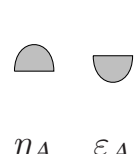

$\eta_{A} \quad \varepsilon_{A}$

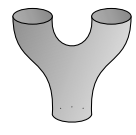

$\mu_{C}$

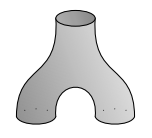

$\Delta_{C}$

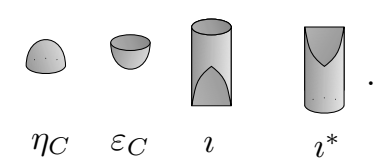

$\imath^{*}$

The global structure of such open-closed cobordisms $M$ is described by specifying two faces $\partial_{0} M$ and $\partial_{1} M$ of $M$ as follows, called the black and the coloured boundary, respectively:

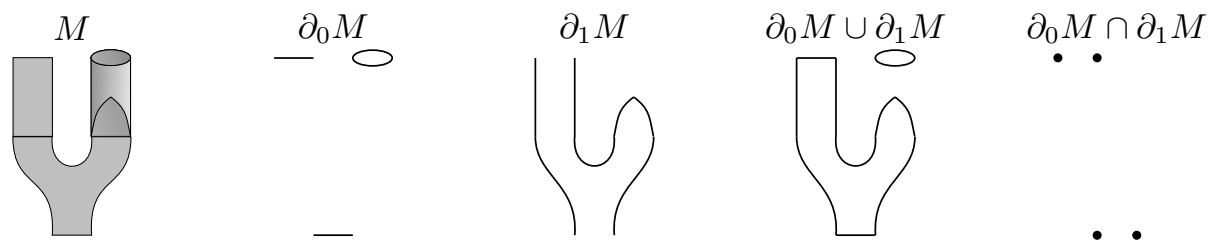

The union $\partial_{0} M \cup \partial_{1} M=\partial M$ is the boundary of $M$ as a topological manifold, and their intersection $\partial_{0} M \cap \partial_{1} M$ is the set of corners of $M$. Open-closed cobordisms are composed by gluing them along their black boundaries.

An open-closed TQFT is a symmetric monoidal functor $Z: \mathbf{2} \mathbf{C o b}^{\text {ext }} \rightarrow \mathbf{V e c t}_{k}$. If we denote by $A:=Z((1))$ the vector space associated with a single arc and by $C:=Z((0))$ the vector space for a single circle, then the relations of $\mathbf{2} \mathbf{C o b}^{\text {ext }}$ are precisely the Moore-Segal relations [16]. They imply that $\left(A, C, \imath, \imath^{*}\right)$ forms what we call a knowledgeable Frobenius algebra, i.e. that $\left(A, \mu_{A}, \eta_{A}, \Delta_{A}, \varepsilon_{A}\right)$ is a symmetric Frobenius algebra, that $\left(C, \mu_{C}, \Delta_{C}, \eta_{C}, \varepsilon_{C}\right)$ is a commutative Frobenius algebra, and that some additional conditions on $\imath$ and $\imath^{*}$ hold. For the details, we refer to Section 2.1 below.

From Bar-Natan's work [5] and from his purely topological proof of the invariance of his tangle homology under Reidemeister moves, it is immediately obvious that an open-closed TQFT is just the appropriate tool for turning Bar-Natan's topological setup into an algebraic tangle homology theory. What remains to be done is to find knowledgeable Frobenius algebras $\left(A, C, \imath, \imath^{*}\right)$ and thereby open-closed TQFTs that satisfy the conditions (1.5) to (1.7). We present examples of such knowledgeable Frobenius algebras $\left(A, C, \imath, \imath^{*}\right)$ for which $C=C_{h, t}$ (Definition 1.1) and for which the resulting tangle homology theories reduce to the link homology theories of Khovanov, Lee, and Bar-Natan when they are evaluated for links. This resolves the first issue, namely to find homology theories for arbitrary oriented tangles (see Theorem 3.37 below).

If we wish to extend Khovanov's Frobenius algebra $C_{0,0}$ (Definition 1.1) with its grading to a knowledgeable Frobenius algebra that is graded, too, then the Cardy condition $\mu_{A} \circ \tau_{A, A^{\circ}} \Delta_{A}=\imath \circ \imath^{*}$ which is a consequence of the following diffeomorphism,

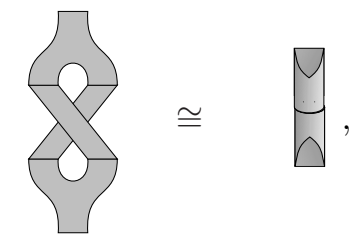

implies that the underlying field has finite characteristic (see Proposition 3.5 below). 


\subsection{Composition of tangles}

The answer to the second question, namely to find an algebraic operation that represents the composition of tangles, is inspired by our recent work [17] on the state sum construction of openclosed TQFTs, i.e. the construction of open-closed TQFTs from triangulations of the open-closed cobordisms. The mechanism by which one can subdivide edges in the black boundary of an openclosed cobordism, provides us with the blue print for an algebraic operation to compose tangles. For example, triangulate the cobordism $\mu_{C}$ of (1.12). Then the three circles in its black boundary are composed from several edges of the triangulation. This composition of the boundary edges to the circle is our guiding example for defining the composition of arcs.

To make this mechanism work, we impose the additional conditions (see Theorem 2.22 of [17] or Proposition 2.7 below) that hold for those open-closed TQFTs that we can construct from a state sum. In particular, these state sum open-closed TQFTs $Z: \mathbf{2} \mathbf{C o b}^{\text {ext }} \rightarrow$ Vect $_{k}$ have an associated knowledgeable Frobenius algebra $\left(A, C, \imath, \imath^{*}\right)$, i.e. $A=Z((1)), C=Z((0))$, etc., for which $A$ is strongly separable ${ }^{5}$ and for which $C$ is the centre of $A$.

If the algebra $A$ is strongly separable, then the element $a=\mu_{A} \circ \Delta_{A} \circ \eta_{A}: k \rightarrow A$ associated with the surface

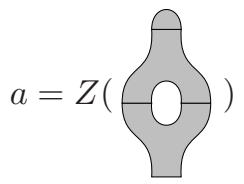

has a convolution inverse $a^{-1}: k \rightarrow A$, i.e. $\mu_{A} \circ\left(a \otimes a^{-1}\right)=\eta_{A}=\mu_{A} \circ\left(a^{-1} \otimes a\right)$. We call $a$ the window element. Both $a$ and $a^{-1}$ are central, i.e. for any linear map $\varphi: A \rightarrow A$, we have $\mu_{A} \circ(a \otimes \varphi)=\mu_{A} \circ(\varphi \otimes a)$. In a strongly separable algebra, multiplication with the inverse of the window element can be used to remove holes (technical term: windows). By this, we mean that

$$
\left.\mu_{A} \circ\left(a^{-1} \otimes Z(\circlearrowleft)\right)\right)=\operatorname{id}_{A}=Z(\square),
$$

i.e. multiplication by $a^{-1}$ on the algebraic side has the same effect as removing a window on the topological side.

If one wishes to compose tangles, say a crossing ( () with an arc ( ) ) in order to get the tangle $(\supset)$, one needs to compose the extended cobordisms that generalize the $S_{(\alpha, j)}$ of (1.2) in a suitable fashion. Let $Z: \mathbf{2 C o b}^{\text {ext }} \rightarrow$ Vect $_{k}$ be an open-closed TQFT that satisfies Bar-Natan's conditions, and let $\left(A, C, \imath, \imath^{*}\right)$ be its associated knowledgeable Frobenius algebra.

For a single crossing $\left(\right.$ (), we have the two smoothings $D_{\emptyset}=$ ) ( and $D_{\{1\}}=\asymp$. With such a crossing ( $($ ) , we associate the 2 -term chain complex of vector spaces

$$
0 \longrightarrow Z\left(D_{\emptyset}\right) \stackrel{Z\left(S_{(\emptyset, 1)}\right)}{\longrightarrow} Z\left(D_{\{1\}}\right) \longrightarrow 0
$$

whose differential is obtained from the saddle $S_{(\emptyset, 1)}$ depicted in (1.3). This saddle is now viewed as an open-closed cobordism, and so it gives a map $Z\left(S_{(\emptyset, 1)}\right): A \otimes A \rightarrow A \otimes A$. To the arc () ), we associate the 1 -term chain complex $0 \rightarrow A \rightarrow 0$.

The tensor product of the two chain complexes is the following 2-term chain complex

$$
0 \longrightarrow Z\left(D_{\emptyset}\right) \otimes A \longrightarrow{ }_{\left(S_{(\emptyset, 1)}\right) \otimes \mathrm{id}_{A}}^{\longrightarrow} Z\left(D_{\{1\}}\right) \otimes A \longrightarrow 0
$$

\footnotetext{
${ }^{5}$ This implies that $A$ is finite-dimensional over $k$ and semisimple.
} 
which can be associated with the following disjoint union of open-closed cobordisms:

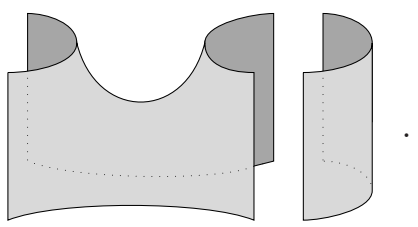

We would like to glue the two open-cobordisms along their coloured boundaries like this,

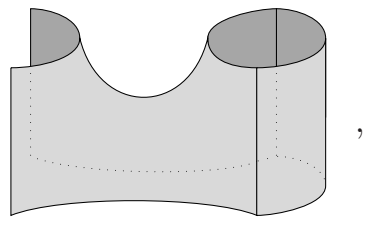

in order to obtain a cobordism from the 0-smoothing ( $) 0$ ) of ( $\gg)$ to the 1-smoothing ( $\gg$ ) of ( $\supset$ ). In general, however, an open-closed TQFT does not have any operation for such a gluing along coloured boundaries.

Our work [17] on the state sum construction of open-closed TQFTs suggests the following solution to this problem. We assume that the open-closed TQFT is a state sum open-closed TQFT (Proposition 2.7 below). We imagine that the composite surface 1.20) is triangulated in such a way that the components of the coloured boundary in (1.20) along which we want to glue, coincide with edges of the triangulation. This implies that the circle in the source of the open-closed cobordism (1.20) is triangulated with two edges and the arc in its target with three edges. Such a triangulation is displayed here:

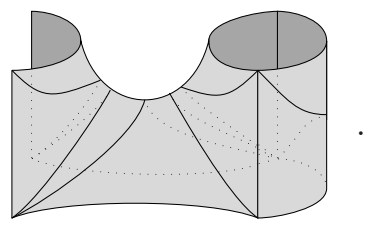

We can now employ the state sum construction presented in [17] in order to compute the linear map $A \otimes C \rightarrow A$ that the open-closed TQFT associates with the open-closed cobordism

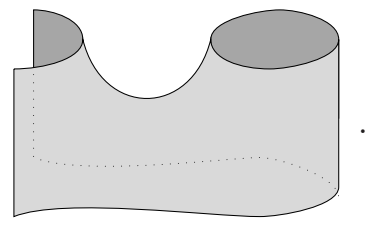

This gives the chain complex $0 \rightarrow A \otimes C \rightarrow A \rightarrow 0$ which relates the two smoothings ()o) and ( $`$ ) of the composite tangle diagram ( $(\supset)$.

We now present an equivalent way of computing the linear map $A \otimes C \rightarrow A$ which makes transparent how this linear map can be computed from the two constituents of (1.19). This procedure does not refer to triangulations or to state sums, and so it can be understood without being familiar with [17]. We nevertheless encourage the reader to study [17] in greater detail and to verify that both procedures indeed result in the same linear map.

In an open-closed TQFT, we cannot compose the two constituents of (1.19) by gluing them along their coloured boundary. We rather pre- and postcompose (1.19) with suitable open-closed 
cobordisms by gluing along their black boundary as follows:

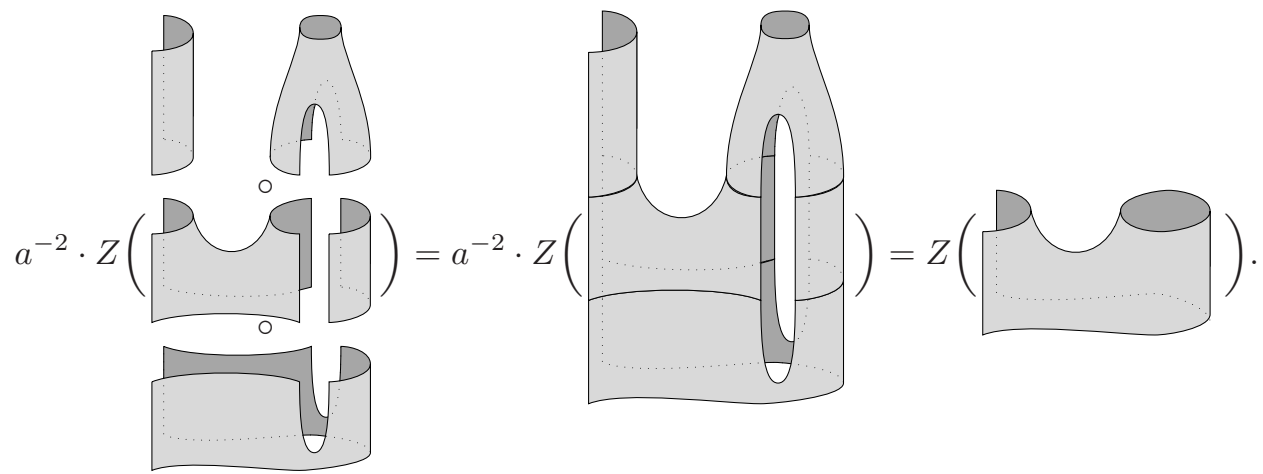

The composition is an open-closed cobordism with two windows, but multiplication by the appropriate power of the inverse window element $a^{-1}$ removes these windows and results in the desired composite open-closed cobordism.

How do we find the appropriate maps
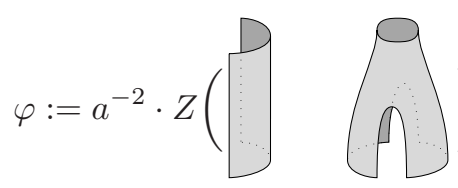

and

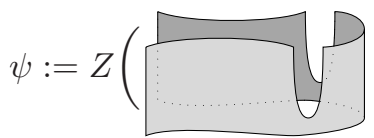

by which to pre- and postcompose? The linear map $Z\left(S_{(\emptyset, 1)}\right): A \otimes A \rightarrow A \otimes A$ associated with the saddle is a morphism of $\left(A^{\otimes 2}, A^{\otimes 2}\right)$-bimodules, and so the 2-term chain complex associated with the crossing is a chain complex of $\left(A^{\otimes 2}, A^{\otimes 2}\right)$-bimodules. Similarly, the algebra $A$ associated with the arc forms an $(A, A)$-bimodule, and so the 1-term chain complex associated with the arc is a chain complex of $(A, A)$-bimodules. In general, for each open end of a tangle, we have one action of $A$.

The linear map associated with the composite (1.22) is the tensor product over $A \otimes A$ of these chain complexes, using the appropriate left- and right-actions of $A \otimes A$ that correspond to the two pairs of open ends of the tangles that are glued. This is completely analogous to the situation for even tangles [14]. The map $\psi$ is the coequalizer that defines the tensor product over $A \otimes A$ in the target of the saddle, whereas the map $\varphi$ is the unique map obtained from the universal property of a similar coequalizer that defines the tensor product over $A \otimes A$ in the source of the saddle because the differential of the chain complex factors through that coequalizer. We make all this precise in Section 4 below.

\subsection{Summary}

In an open-closed TQFT, we have operations for putting the building blocks of 1.12) on top of each other by gluing them along their black boundaries (composition) and for putting them next to each other by taking their disjoint unions (tensor product), but there is no elementary operation for gluing them 'sideways' along their coloured boundaries. If in addition $A$ is strongly separable and if $C=Z(A)$ with the canonical inclusion $\imath: C \rightarrow A$, then the above prescription can be viewed as an additional operation that allows us to glue open-closed cobordisms along their coloured boundaries.

If $\left(A, C, \imath, \imath^{*}\right)$ is a state sum knowledgeable Frobenius algebra with $A$ strongly separable, and $C=Z(A)$, then $C$ is strongly separable, too. The commutative Frobenius algebra $C_{0,1}$ (Definition 1.11) of Lee in characteristic other than 2 and $C_{1,0}$ of Bar-Natan in any characteristic are strongly separable, and so our strategy for composing tangles works in these cases. Khovanov's Frobenius algebra $C_{0,0}$ in any characteristic and Lee's Frobenius algebra in characteristic 2 are not semisimple and both fail to yield a tangle homology theory that is well behaved under tangle composition defined in this way. This is no surprise. The non-semisimplicity of Khovanov's 
Frobenius algebra is closely related to the fact that it can detect global properties of the tangle that cannot be easily computed locally, i.e. from the constituents of the tangle.

But does this mean that our method of composing tangles rules out the most interesting example, namely Khovanov's? No, it does not. There is still a way out. It is known, for example, from the work of Lee and Rasmussen $[12,18]$ and of Turner [13] that for links, the chain complex and homology for Khovanov's Frobenius algebra can be recovered from a filtration on the chain complex of Lee or Bar-Natan, respectively, by computing the $E_{0}$ - and $E_{1}$-pages of a certain spectral sequence. When we extend this method of computing Khovanov's chain complex from links to tangles, there are additional conditions to satisfy, and an analogous spectral sequence only exists over fields of finite characteristic (see Example 3.13 Theorem [3.20] and Examples 3.22 to 3.25]). These extensions are based on strongly separable algebras, and they are well behaved under tangle composition. Already the simplest example in characteristic 2 (Example 3.13) carries a filtration sophisticated enough to recover our tangle extension of characteristic 2 Khovanov homology with all its non-trivial properties (Theorem 3.42 and Remark 3.45 below).

The tangle homology presented in this article then has the following desirable properties:

1. The tangle homology behaves well with respect to composition.

2. It respects the monoidal structure of the category of tangles.

3. It is rich enough to recover the finite characteristic Khovanov homology for links as well as Rasmussen's $s$-invariant.

Our use of open-closed TQFTs in order to define a tangle homology theory is by no means the most general conceivable approach. For example, in an open-closed TQFT, all the openclosed cobordisms displayed in (1.12) have algebraic operations associated with them although only the composites displayed in (3.48) to 3.53) below are needed in order to define the chain complexes. This is in analogy to the observation that in Khovanov's link homology theory, the unit and counit of the commutative Frobenius algebra are not needed to define the chain complex. Notice, however, that both Bar-Natan's [5] and our proof (Section 4.3 below) of Reidemeister move invariance still need some of the additional cobordisms. We have chosen to work with open-closed TQFTs mainly because the extension of a 2-dimensional TQFT to an open-closed TQFT is much better understood than the corresponding question for the Temperley-Lieb 2-category. Notice also that in any tangle cobordism theory, the object associated with the unknot, i.e. in our case the homology of the 1-term complex $0 \rightarrow C \rightarrow 0$ which is just $C$, automatically carries the structure of a commutative Frobenius algebra. Since we work with open-closed TQFTs, the object associated with the arc, i.e. the identity tangle on a single point, which is the homology of $0 \rightarrow A \rightarrow 0$ and thereby just $A$, forms a symmetric Frobenius algebra. This is sufficient to yield an algebraic model for the Temperley-Lieb 2-category, but presumably not necessary.

\section{Preliminaries}

In this section, we introduce the notion of a knowledgeable Frobenius algebra. This is the algebraic structure that is needed to classify open-closed TQFTs. We then summarize the definitions and the main properties of the commutative and anti-commutative cubes of graded and filtered vector spaces that are used to define Khovanov's, Lee's and Bar-Natan's chain complexes.

\subsection{Knowledgeable Frobenius algebras and open-closed TQFTs}

This section is a brief summary of the relevant definitions and results. For more details, we refer to $[15,17]$.

Definition 2.1. Let $k$ be a field. 
- A Frobenius algebra $(A, \mu, \eta, \Delta, \varepsilon)$ is an associative algebra $(A, \mu, \eta)$ with multiplication $\mu: A \otimes A \rightarrow A$ and unit $\eta: k \rightarrow A$ that forms a coassociative coalgebra $(A, \Delta, \varepsilon)$ with comultiplication $\Delta: A \rightarrow A \otimes A$ and counit $\varepsilon: A \rightarrow k$ such that the following condition holds,

$$
\left(\operatorname{id}_{A} \otimes \mu\right) \circ\left(\Delta \otimes \operatorname{id}_{A}\right)=\Delta \circ \mu=\left(\mu \otimes \operatorname{id}_{A}\right) \circ\left(\operatorname{id}_{A} \otimes \Delta\right) .
$$

- A Frobenius algebra $(A, \mu, \eta, \Delta, \varepsilon)$ is called symmetric if $\varepsilon \circ \mu \circ \tau_{A, A}=\varepsilon \circ \mu$. It is called commutative if $\mu \circ \tau_{A, A}=\mu$. We have used the notation $\tau_{A, A}: A \otimes A \rightarrow A \otimes A, a \otimes b \mapsto b \otimes a$.

- In a symmetric Frobenius algebra $(A, \mu, \eta, \Delta, \varepsilon)$, we call the element $a=\mu \circ \Delta \circ \eta: k \rightarrow A$ the window element.

- An algebra $(A, \mu, \eta)$ is called strongly separable if the canonical bilinear form

$$
g_{\text {can }}: A \otimes A \rightarrow k, \quad a \otimes b \mapsto \operatorname{tr}_{A}\left(L_{a} \circ L_{b}\right)
$$

is non-degenerate. Here we have written $L: A \rightarrow \operatorname{End}(A), a \mapsto L_{a}$ with $L_{a}: A \rightarrow A, b \mapsto$ $\mu(a \otimes b)$ for the left-regular representation.

- A homomorphism of Frobenius algebras $f: A \rightarrow A^{\prime}$ is a linear map which is both a homomorphism of unital algebras and of counital coalgebras.

Every strongly separable algebra is finite-dimensional and semisimple. The converse implication holds in characteristic 0 but not in general in finite characteristic.

A symmetric Frobenius algebra is strongly separable if and only if the window element is convolution invertible, i.e. if there exists some $a^{-1}: k \rightarrow A$ such that $\mu \circ\left(a \otimes a^{-1}\right)=\eta=\mu \circ\left(a^{-1} \otimes a\right)$. The window element is central, i.e. $\mu \circ(\varphi \otimes a)=\mu \circ(a \otimes \varphi)$ for all linear maps $\varphi: A \rightarrow A$, and so is its inverse when it exists. We write $a \cdot \operatorname{id}_{A}:=\mu_{A} \circ\left(a \otimes \operatorname{id}_{A}\right)$, etc..

The Frobenius algebra $C_{h, t}$ of Definition 1.1 has the window element $a=(\mu \circ \Delta \circ \eta)(1)=2 x-h$ which satisfies $a^{2}=\left(h^{2}+4 t\right) \cdot 1$, and so $C_{h, t}$ is strongly separable if and only if $h^{2}+4 t \neq 0$. In particular, Khovanov's Frobenius algebra $C_{0,0}$ is not strongly separable; Lee's $C_{0,1}$ is strongly separable if and only if char $k \neq 2$; and Bar-Natan's $C_{1,0}$ is strongly separable in any characteristic.

Definition 2.2. A knowledgeable Frobenius algebra $\left(A, C, \imath, \imath^{*}\right)$ consists of,

- a symmetric Frobenius algebra $A=\left(A, \mu_{A}, \eta_{A}, \Delta_{A}, \varepsilon_{A}\right)$,

- a commutative Frobenius algebra $C=\left(C, \mu_{C}, \eta_{C}, \Delta_{C}, \varepsilon_{C}\right)$,

- linear maps $\imath: C \rightarrow A$ and $\imath^{*}: A \rightarrow C$,

such that $\imath: C \rightarrow A$ is a homomorphism of algebras and the following conditions hold,

$$
\begin{aligned}
\mu_{A} \circ\left(\imath \otimes \mathrm{id}_{A}\right) & =\mu_{A} \circ \tau_{A, A} \circ\left(\imath \otimes \mathrm{id}_{A}\right) & & \text { (knowledge), } \\
\varepsilon_{C} \circ \mu_{C} \circ\left(\operatorname{id}_{C} \otimes \imath^{*}\right) & =\varepsilon_{A} \circ \mu_{A} \circ\left(\imath \otimes \operatorname{id}_{A}\right) & & \text { (duality), } \\
\mu_{A} \circ \tau_{A, A} \circ \Delta_{A} & =\imath \circ \imath^{*} & & \text { (Cardy condition). }
\end{aligned}
$$

The defining relations of a knowledgeable Frobenius algebra are precisely the Moore-Segal relations [16]. A homomorphism of knowledgeable Frobenius algebras $f:\left(A, C, \imath, \imath^{*}\right) \rightarrow\left(A^{\prime}, C^{\prime}, \imath^{\prime}, \imath^{\prime *}\right)$ is a pair $f=\left(f_{1}, f_{2}\right)$ such that $f_{1}: A \rightarrow A^{\prime}$ and $f_{2}: C \rightarrow C^{\prime}$ are homomorphisms of Frobenius algebras and such that $f_{1} \circ \imath=\imath^{\prime} \circ f_{2}$ and $f_{2} \circ \imath^{*}=\imath^{\prime *} \circ f_{1}$. We denote the category of knowledgeable Frobenius algebras and their homomorphisms by $\mathbf{K}-\mathbf{F r o b}\left(\operatorname{Vect}_{k}\right)$.

The category $\mathbf{K}-\mathbf{F r o b}\left(\mathbf{V e c t}_{k}\right)$ is a symmetric monoidal category. The tensor product of two knowledgeable Frobenius algebras $\mathbb{A}=\left(A, C, \imath, \imath^{*}\right)$ and $\mathbb{A}^{\prime}=\left(A^{\prime}, C^{\prime}, \imath^{\prime}, \imath^{\prime *}\right)$ is $\mathbb{A} \otimes \mathbb{A}^{\prime}=\left(A \otimes_{k}\right.$ $\left.A^{\prime}, C \otimes_{k} C^{\prime}, \imath \otimes \imath^{\prime}, \imath^{*} \otimes \imath^{\prime *}\right)$. The unit of the monoidal structure is $\left(k, k, \operatorname{id}_{k}, \operatorname{id}_{k}\right)$ where $k$ is equipped with the trivial Frobenius algebra structure $\Delta_{k}(1)=1 \otimes 1$ and $\varepsilon_{k}(1)=1$. The associativity and unit constraints and the symmetric braiding are inherited from Vect $_{k}$. 
There is a category $\mathbf{2} \mathbf{C o b}^{\text {ext }}$ of open-closed cobordisms which generalizes the familiar category 2Cob of 2-dimensional cobordisms from manifolds with boundary to manifolds with corners that have a particular global structure as sketched in 1.13). The objects of $\mathbf{2} \mathbf{C o b}^{\text {ext }}$ are sequences $\left(m_{1}, \ldots, m_{k}\right) \in\{0,1\}^{k}, k \in \mathbb{N}_{0}$, which specify a particular sequence of circles $\left(m_{j}=0\right)$ and $\operatorname{arcs}\left(m_{j}=1\right)$. The morphisms are the equivalence classes of suitable 2-manifolds with corners up to orientation-preserving diffeomorphisms that restrict to the identity on the black boundary. There exists a generators and relations description for the morphisms of $\mathbf{2} \mathbf{C o b}{ }^{\mathrm{ext}}$. The generators are the equivalence classes of the open-closed cobordisms of (1.12), and the relations among the morphisms of $\mathbf{2} \mathbf{C o b}^{\text {ext }}$ are precisely the defining equations of a knowledgeable Frobenius algebra. We recommend that the reader draw the pictures corresponding to all equations that appear in this section. The main result of [15] can be stated as follows.

Theorem 2.3 (see [15]). The category $2 \mathbf{C o b}^{\text {ext }}$ of open-closed cobordisms is the strict symmetric monoidal category freely generated by a knowledgeable Frobenius algebra object $\left(A, C, \imath, \imath^{*}\right)$. Here, $A=(1)$ represents the diffeomorphism type of the arc; $C=(0)$ is the one of the circle; and the structure maps of the knowledgeable Frobenius algebra object are the equivalence classes of the open-closed cobordisms depicted in (1.12).

Definition 2.4. An open-closed Topological Quantum Field Theory (TQFT) is a symmetric monoidal functor $Z: \mathbf{2} \mathbf{C o b}^{\text {ext }} \rightarrow$ Vect $_{k}$. A homomorphism of open-closed TQFTs is a monoidal natural transformation between such functors. We denote the category of open-closed TQFTs and their homomorphisms by

$$
\operatorname{OC-TQFT}\left(\operatorname{Vect}_{k}\right):=\operatorname{Symm}-\operatorname{Mon}\left(2 \operatorname{Cob}^{\text {ext }}, \operatorname{Vect}_{k}\right)
$$

Corollary 2.5. There is an equivalence of symmetric monoidal categories

$$
\operatorname{OC-TQFT}\left(\operatorname{Vect}_{k}\right) \underset{G}{\rightleftarrows} \mathbf{K}-\mathbf{F r o b}\left(\operatorname{Vect}_{k}\right)
$$

between the category of open-closed TQFTs and that of knowledgeable Frobenius algebras. Given an open-closed TQFT $Z$, we call $F(Z)$ the associated knowledgeable Frobenius algebra. It is determined up to isomorphism of knowledgeable Frobenius algebras by $\left(A, C, \imath, \imath^{*}\right)$ where $A=$ $Z((1)), C=Z((0))$, and the structure maps are the images of the open-closed cobordisms of (1.12) under $Z$.

In order to turn Bar-Natan's 'picture world' [5] into algebra, we show below that it is sufficient if the corresponding knowledgeable Frobenius algebra $\left(A, C, \imath, \imath^{*}\right)$ has a commutative Frobenius algebra $\left(C, \mu_{C}, \eta_{C}, \Delta_{C}, \varepsilon_{C}\right)$ that satisfies Bar-Natan's conditions.

\section{Definition 2.6.}

1. A knowledgeable Frobenius algebra $\left(A, C, \imath, \imath^{*}\right)$ is said to satisfy Bar-Natan's conditions if $C$ satisfies them (Definition 1.2).

2. An open-closed TQFT is said to satisfy Bar-Natan's conditions if its associated knowledgeable Frobenius algebra does so.

\subsection{Strongly separable algebras and state sum TQFTs}

Let us now focus on strongly separable symmetric Frobenius algebras. These are used to construct state sum open-closed TQFTs in [17]. From every such algebra, one can construct an openclosed TQFT which is described by the knowledgeable Frobenius algebra defined in the following proposition.

Proposition 2.7 (see Theorem 2.22 of [17]). Let $\left(A, \mu_{A}, \eta_{A}, \Delta_{A}, \varepsilon_{A}\right)$ be a strongly separable symmetric Frobenius algebra with window element $a$. Then

$$
p:=\left(a^{-1} \cdot \operatorname{id}_{A}\right) \circ \mu_{A} \circ \tau_{A, A} \circ \Delta_{A}: A \rightarrow A
$$


forms an idempotent whose image is the centre of $A$, i.e. $p(A)=Z(A)$.

In this case, there is a knowledgeable Frobenius algebra $\left(A, C, \imath, \imath^{*}\right)$ with $C:=Z(A)$, equipped with the commutative Frobenius algebra structure $\left(C, \mu_{C}, \eta_{C}, \Delta_{C}, \varepsilon_{C}\right)$ given by

$$
\begin{aligned}
\mu_{C} & =p \circ \mu_{A}, \\
\eta_{C} & =p \circ \eta_{A}, \\
\Delta_{C} & =(p \otimes p) \circ \Delta_{A} \circ\left(a \cdot \operatorname{id}_{A}\right), \\
\varepsilon_{C} & =\varepsilon_{A} \circ\left(a^{-1} \cdot \operatorname{id}_{A}\right),
\end{aligned}
$$

and with the canonical inclusion $\imath: C \rightarrow A$ and the map $\imath^{*}=\left(a \cdot \operatorname{id}_{A}\right) \circ p$.

Definition 2.8. A state sum knowledgeable Frobenius algebra is a knowledgeable Frobenius algebra that arises from a strongly separable symmetric Frobenius algebra by the construction of Proposition 2.7. A state sum open-closed TQFT Z: $\mathbf{2 C o b}^{\text {ext }} \rightarrow$ Vect $_{k}$ is an open-closed TQFT whose associated knowledgeable Frobenius algebra is a state sum knowledgeable Frobenius algebra.

In order to compose tangles, we need some idempotents defined in the subsequent proposition. Let $\left(A, \mu_{A}, \eta_{A}, \Delta_{A}, \varepsilon_{A}\right)$ be a symmetric Frobenius algebra. For $j \in \mathbb{N}$, we denote by

$$
\mu_{A}^{(j+1)}:=\mu_{A} \circ\left(\mu_{A}^{(j)} \otimes \operatorname{id}_{A}\right), \quad \mu_{A}^{(2)}:=\mu_{A}, \quad \mu_{A}^{(1)}:=\operatorname{id}_{A}
$$

and by

$$
\Delta_{A}^{(j+1)}:=\left(\Delta_{A}^{(j)} \otimes \operatorname{id}_{A}\right) \circ \Delta_{A}, \quad \Delta_{A}^{(2)}:=\Delta_{A}, \quad \Delta_{A}^{(1)}:=\operatorname{id}_{A}
$$

the iterated multiplication and comultiplication. We also write $A^{\otimes(j+1)}:=A^{\otimes j} \otimes A, A^{\otimes 1}:=A$ and $A^{\otimes 0}:=\mathbb{1}$, and $a^{j+1} \cdot \operatorname{id}_{A}:=\left(a^{j} \cdot \operatorname{id}_{A}\right) \circ\left(a \cdot \operatorname{id}_{A}\right)$ and $a^{0} \cdot \operatorname{id}_{A}:=\operatorname{id}_{A}$.

Proposition 2.9. Let $\left(A, \mu_{A}, \eta_{A}, \Delta_{A}, \varepsilon_{A}\right)$ be a strongly separable symmetric Frobenius algebra with window element $a$, and let $p$ be as in Proposition 2.7 Then the linear maps,

$$
\begin{aligned}
P_{j \ell} & :=\Delta^{(j)} \circ\left(a^{-(j-1)} \cdot \mathrm{id}_{A}\right) \circ \mu^{(\ell)}: A^{\otimes \ell} \rightarrow A^{\otimes j}, \\
Q_{j \ell}: & =\Delta^{(j)} \circ\left(a^{-(j-1)} \cdot \operatorname{id}_{A}\right) \circ p \circ \mu^{(\ell)}: A^{\otimes \ell} \rightarrow A^{\otimes j},
\end{aligned}
$$

satisfy

$$
P_{j \ell} \circ P_{\ell m}=P_{j m} \quad \text { and } \quad Q_{j \ell} \circ Q_{\ell m}=Q_{j m}
$$

for all $j, \ell, m \in \mathbb{N}$. In particular, $P_{j j}$ and $Q_{j j}$ are idempotents, and we have $P_{11}=\mathrm{id}_{A}$ and $Q_{11}=p$. There are isomorphisms,

$$
P_{j j}\left(A^{\otimes j}\right) \cong A \quad \text { and } \quad Q_{j j}\left(A^{\otimes j}\right) \cong p(A),
$$

given by $P_{1 j}$ and $Q_{1 j}$, respectively, whose inverses are $P_{j 1}$ and $Q_{j 1}$, respectively.

\subsection{Tensor products and coequalizers}

The images of these idempotents can be characterized by the following universal properties that may be more familiar to the reader.

Proposition 2.10. Let $\left(A, \mu_{A}, \eta_{A}, \Delta_{A}, \varepsilon_{A}\right)$ be a strongly separable symmetric Frobenius algebra, and let $p=Q_{11}$ and $P_{22}$ be as above.

1. The linear map $p: A \rightarrow p(A)$ is the coequalizer

$$
A \otimes A \underset{\mu_{A} \circ \tau_{A, A}}{\stackrel{\mu_{A}}{\rightleftharpoons}} A \stackrel{p}{\longrightarrow} p(A) .
$$


2. The linear map $P_{22}: A \otimes A \rightarrow P_{22}(A \otimes A)$ is the coequalizer

$$
A \otimes A \otimes A \underset{\mu_{A} \otimes \mathrm{id}_{A}}{\stackrel{\operatorname{id}_{A} \otimes \mu_{A}}{\longrightarrow}} A \otimes A \stackrel{P_{22}}{\longrightarrow} P_{22}(A \otimes A) .
$$

This means that $p(A) \cong A /[A, A]$ and that $P_{22}(A \otimes A) \cong A \otimes_{A} A$ where $A$ is viewed as an $(A, A)$-bimodule.

Proof. We prove this proposition for algebra objects in an arbitrary abelian symmetric monoidal category $\mathcal{C}$. Given the idempotent (2.8), we denote its image splitting by $p=\operatorname{im} p \circ \operatorname{coim} p$ with im $p: p(A) \rightarrow A$ and coim $p: A \rightarrow p(A)$. In the abelian category $\mathcal{C}$, the idempotent $p$ splits, and so coim $p \circ \operatorname{im} p=\operatorname{id}_{p(A)}$.

We assume that $\left(A, \mu_{A}, \eta_{A}, \Delta_{A}, \varepsilon_{A}\right)$ is a strongly separable symmetric Frobenius algebra object in some abelian symmetric monoidal category $\mathcal{C}$ and prove the following claim:

1. The coimage coim $p: A \rightarrow p(A)$ is the coequalizer

$$
A \otimes A \underset{\mu_{A} \circ \tau_{A, A}}{\stackrel{\mu_{A}}{\longrightarrow}} A \stackrel{\operatorname{coim} p}{\longrightarrow} p(A) .
$$

2. The coimage coim $P_{22}: A \otimes A \rightarrow P_{22}(A \otimes A)$ is the coequalizer

$$
(A \otimes A) \otimes A \underset{\left(\mathrm{id}_{A} \otimes \mu_{A}\right) \circ \alpha_{A, A, A}}{\stackrel{\mu_{A} \otimes \mathrm{id}_{A}}{\longrightarrow}} A \otimes A \stackrel{\operatorname{coim} P_{22}}{\longrightarrow} P_{22}(A \otimes A) .
$$

In order to prove (1.), note that since $\operatorname{coim} p=\operatorname{coim} p \circ p$, the morphism coim $p$ satisfies coim $p \circ \mu_{A}=\operatorname{coim} p \circ \mu_{A} \circ \tau_{A, A}$. Given any morphism $f: A \rightarrow B$ such that $f \circ \mu_{A}=f \circ \mu_{A} \circ \tau_{A, A}$, there is a morphism $\varphi: p(A) \rightarrow B$ given by $\varphi:=f \circ \operatorname{im} p$ such that

$$
\varphi \circ \operatorname{coim} p=f \circ p=f \circ \mu_{A} \circ \tau_{A, A} \circ \Delta_{A} \circ\left(a^{-1} \cdot \operatorname{id}_{A}\right)=f \circ \mu_{A} \circ \Delta_{A} \circ\left(a^{-1} \cdot \operatorname{id}_{A}\right)=f .
$$

If $\psi: p(A) \rightarrow B$ also satisfies $f=\psi \circ \operatorname{coim} p$, then $\varphi=f \circ \operatorname{im} p=\psi \circ \operatorname{id}_{p(A)}=\psi$, and so $\varphi$ is unique with that property.

In order to prove (2.), note that since coim $P_{22}=$ coim $P_{22} \circ P_{22}$, and because of associativity, we have coim $P_{22} \circ\left(\mu_{A} \otimes \operatorname{id}_{A}\right)=$ coim $P_{22} \circ\left(\operatorname{id}_{A} \otimes \mu_{A}\right) \circ \alpha_{A, A, A}$. Given any morphism $f: A \otimes A \rightarrow B$ such that $f \circ\left(\mu_{A} \otimes \operatorname{id}_{A}\right)=f \circ\left(\operatorname{id}_{A} \otimes \mu_{A}\right) \circ \alpha_{A, A, A}$, there is a morphism $\varphi:=f \circ \operatorname{im} P_{22}: P_{22}(A \otimes A) \rightarrow B$ such that

$$
\begin{aligned}
\varphi \circ \operatorname{coim} P_{22} & =f \circ P_{22}=f \circ\left(\mu_{A} \otimes \operatorname{id}_{A}\right) \circ \alpha_{A, A, A}^{-1} \circ\left(\operatorname{id}_{A} \otimes \Delta_{A}\right) \circ\left(\operatorname{id}_{A} \otimes\left(a^{-1} \cdot \operatorname{id}_{A}\right)\right) \\
& =f \circ\left(\operatorname{id}_{A} \otimes\left(\mu_{A} \circ \Delta_{A} \circ\left(a^{-1} \cdot \operatorname{id}_{A}\right)\right)\right)=f .
\end{aligned}
$$

If $\psi: P_{22}(A \otimes A) \rightarrow B$ also satisfies $\psi \circ$ coim $P_{22}=f$, then $\varphi=f \circ$ im $P_{22}=\psi \circ$ coim $P_{22} \circ$ im $P_{22}=$ $\psi$, and so $\varphi$ is unique with that property.

Using the fact that $P_{22}$ is a morphism of $(A, A)$-bimodules and that $A \otimes_{A} A \cong A$ as $(A, A)$ bimodules, one can obtain similar characterizations for the images $P_{j j}\left(A^{\otimes j}\right)$ and $Q_{j j}\left(A^{\otimes j}\right)$ for all $j \in \mathbb{N}$. Note that the map $\varphi$ of (1.24) in the introduction is $\varphi=\mathrm{id}_{A} \otimes Q_{21}$ whereas $\psi=P_{13}$.

The remainder of the present subsection is merely a 'wild' combination of the above results which introduces a notation relevant to Section 4 below. The reader may wish to skip this material on first reading.

The $k$-fold tensor product $M:=A^{\otimes k}, k \in \mathbb{N}$, forms both a left $A$-module and a right $A$ module in $k$ different ways, by acting with $A$ from the left or from the right on the $j$-th tensor factor, $j \in\{1, \ldots, k\}$. We denote the left actions by $\lambda_{j}: A \otimes M \rightarrow M$ and the right actions by 
$\rho_{j}: M \otimes A \rightarrow A$, respectively. We introduce the following notation for the coequalizer in Vect ${ }_{k}$ that 'coequalizes' the $j$-th left action with the $\ell$-th right action, $j, \ell \in\{1, \ldots, k\}$ :

$$
A \otimes M \underset{\lambda_{j}}{\stackrel{\rho_{\ell} \circ \tau_{A, M}}{\longrightarrow}} M \stackrel{\rho_{\ell} \otimes_{\lambda_{j}}}{\longrightarrow} \rho_{\ell} \otimes_{\lambda_{j}}(M) .
$$

We note that $\rho_{\ell} \otimes_{\lambda_{j}}(M)$ is an $\left(A^{\otimes(k-1)}, A^{\otimes(k-1)}\right)$-bimodule whose associated $A$-actions we denote by $\lambda_{1}, \ldots, \lambda_{j-1}, \lambda_{j+1}, \ldots, \lambda_{k}$ and $\rho_{1}, \ldots, \rho_{\ell-1}, \rho_{\ell+1}, \ldots, \rho_{k}$, respectively. For $j \neq r, \ell \neq s$, taking these coequalizers commutes, and we have canonical isomorphisms

$$
\rho_{\ell} \otimes_{\lambda_{j}}\left(\rho_{s} \otimes_{\lambda_{r}}(M)\right) \cong \rho_{s} \otimes_{\lambda_{r}}\left(\rho_{\ell} \otimes_{\lambda_{j}}(M)\right)
$$

of $\left(A^{\otimes(k-2)}, A^{\otimes(k-2)}\right)$-bimodules.

Corollary 2.11. Let $\left(A, \mu_{A}, \eta_{A}, \Delta_{A}, \varepsilon_{A}\right)$ be a strongly separable symmetric Frobenius algebra, $M:=A^{\otimes k}, k \in \mathbb{N}$, and denote the left $A$ - and right $A$-actions on $M$ as above.

1. $P_{1 k}: M \rightarrow A$ is the composition of coequalizer maps

$$
P_{1 k}={ }_{\rho_{1}} \otimes_{\lambda_{2}} \circ_{\rho_{2}} \otimes_{\lambda_{3}} \circ \cdots \circ \rho_{\rho_{k-1}} \otimes_{\lambda_{k}}
$$

in $\operatorname{Vect}_{k}$.

2. $Q_{1 k}: M \rightarrow p(A)$ is the composition of coequalizer maps

$$
Q_{1 k}=\rho_{k} \otimes_{\lambda_{1}} \circ P_{1 k}
$$

in $\operatorname{Vect}_{k}$.

This result allows us to characterize the map $\psi$ of 1.24) in the introduction by a universal property as soon as we have organized the tensor factors and the associated left and right actions accordingly.

For permutations $\sigma, \tau \in S_{k}$, we define

$$
P_{1 k}^{(\sigma, \tau)}:=\rho_{\tau(1)} \otimes_{\lambda_{\sigma(2)}} \rho_{\rho_{\tau(2)}} \otimes_{\lambda_{\sigma(3)}} \circ \cdots \circ \rho_{\rho_{\tau(k-1)}} \otimes_{\lambda_{\sigma(k)}}
$$

and

$$
Q_{1 k}^{(\sigma, \tau)}:=\rho_{\tau(k)} \otimes_{\lambda_{\sigma(1)}} \circ P_{1 k}^{(\sigma, \tau)} .
$$

Consider a linear map $f: M \rightarrow M, M:=A^{\otimes k}, k \in \mathbb{N}$, that satisfies

$$
\lambda_{\sigma(j)} \circ\left(\operatorname{id}_{A} \otimes f\right)=f \circ \lambda_{j} \quad \text { and } \quad \rho_{\tau(\ell)} \circ\left(f \otimes \operatorname{id}_{A}\right)=f \circ \rho_{\ell},
$$

for all $j, \ell \in\{1, \ldots, k\}$, for some permutations $\sigma, \tau \in S_{k}$. We call $f$ a $(\sigma, \tau)$-morphism of $\left(A^{\otimes k}, A^{\otimes k}\right)$-bimodules. In fact, $f$ is an honest morphism of $\left(A^{\otimes k}, A^{\otimes k}\right)$-bimodules as soon as one defines the left and right actions of $A^{\otimes k}$ on its target by taking the actions on the source and pre-composing them with the permutations $\sigma$ and $\tau$, respectively. Our prime examples of $(\sigma, \tau)$-morphisms of bimodules are the maps

$$
\left(\mu_{A} \otimes \operatorname{id}_{A}\right) \circ\left(\operatorname{id}_{A} \otimes \tau_{A, A}\right) \circ\left(\Delta_{A} \otimes \operatorname{id}_{A}\right): A \otimes A \rightarrow A \otimes A
$$

with $\sigma=$ id and $\tau=(12)$ in cycle notation, and

$$
\left(\operatorname{id}_{A} \otimes \mu_{A}\right) \circ\left(\tau_{A, A} \otimes \operatorname{id}_{A}\right) \circ\left(\operatorname{id}_{A} \otimes \Delta_{A}\right): A \otimes A \rightarrow A \otimes A
$$

with $\sigma=(12)$ and $\tau=$ id. These maps can be obtained from the open-closed cobordisms depicted in (3.48) and (3.49). 
If $f: M \rightarrow M$ is a $(\sigma, \tau)$-morphism of $\left(A^{\otimes k}, A^{\otimes k}\right)$-bimodules, then for $j, \ell \in\{1, \ldots, k\}$, the composition $\rho_{\tau(\ell)} \otimes_{\lambda_{\sigma(j)}}$ of factors through the coequalizer ${ }_{\rho_{\ell}} \otimes_{\lambda_{j}}$ and uniquely defines a map $\widehat{f}$ such that the diagram

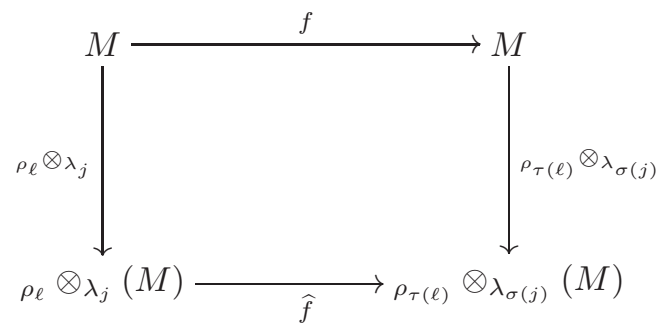

commutes. Iterating this result, we obtain:

Corollary 2.12. Let $\left(A, \mu_{A}, \eta_{A}, \Delta_{A}, \varepsilon_{A}\right)$ be a strongly separable symmetric Frobenius algebra, $M:=A^{\otimes k}, k \in \mathbb{N}$, and let $f: M \rightarrow M$ be a $(\sigma, \tau)$-morphism of $\left(A^{\otimes k}, A^{\otimes k}\right)$-bimodules for some permutations $\sigma, \tau \in S_{k}$.

- The map $\widehat{f}:=P_{1 k}^{(\sigma, \tau)} \circ f \circ P_{k 1}$ is the unique linear map such that the diagram

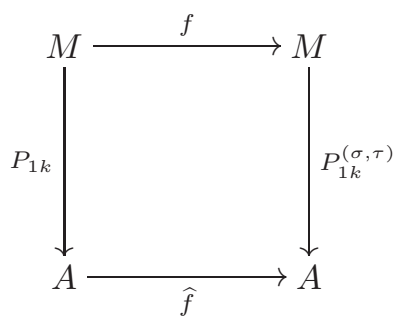

commutes.

- The map $\widehat{f}:=Q_{1 k}^{(\sigma, \tau)} \circ f \circ Q_{k 1}$ is the unique linear map such that the diagram

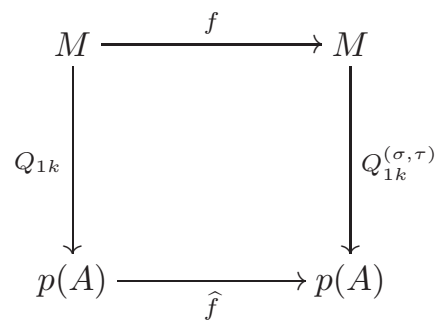

commutes.

Proof. This result follows from (2.17).

This result is the origin of the map $\varphi$ in (1.24) in the introduction. The $Q_{21}$ in $\varphi=\operatorname{id}_{A} \otimes Q_{21}$ is the first factor in such a composition $\widehat{f}$.

\subsection{Gradings and filtrations}

A $k$-vector space $V$ is called graded if it is of the form $V \cong \bigoplus_{j \in \mathbb{Z}} V^{j}$. We say that a vector $v \in V^{j}$ is homogeneous of degree $\operatorname{deg}(v)=j \in \mathbb{Z}$. If both $V$ and $W$ are graded vector spaces, then their tensor product $V \otimes W$ is graded with $(V \otimes W)^{j}=\bigoplus_{\ell \in \mathbb{Z}} V^{\ell} \otimes W^{j-\ell}$. The underlying field $k$, i.e. the unit object of the monoidal structure of $\operatorname{Vect}_{k}$, is considered graded with every vector homogeneous of degree 0, i.e. $k=k^{0}$. A linear map $f: V \rightarrow W$ is said to be graded of degree 
$\ell \in \mathbb{Z}$ if $f\left(V^{j}\right) \subseteq W^{j+\ell}$ for all $j \in \mathbb{Z}$. In this case, we write $\operatorname{deg}(f)=\ell$. For the composition of graded linear maps $f: V \rightarrow W$ and $g: W \rightarrow U$, we have $\operatorname{deg}(g \circ f)=\operatorname{deg}(g)+\operatorname{deg}(f)$. If $V$ is a graded vector space, we define for each $j \in \mathbb{Z}$ a graded vector space $V\{j\}$ by $(V\{j\})^{\ell}:=V^{\ell-j}$. The operator $\{j\}$ is called the grading shift by $j$. If the linear map $f: V \rightarrow W$ is graded of degree $\ell$, then the map $f: V \rightarrow W\{-\ell\}$ is graded of degree 0 .

A $k$-vector space $V$ is called filtered if there is a family $\left\{F^{j} V\right\}_{j \in \mathbb{Z}}$ of subspaces $F^{j} V \subseteq V$ such that $F^{j+1} V \subseteq F^{j} V$ for all $j \in \mathbb{Z}$. The filtration is said to be bounded if there exist some $j_{0}, j_{1} \in \mathbb{Z}$ with $F^{j_{0}} V=V$ and $F^{j_{1}} V=\{0\}$. We say that a vector $v \in V$ is of $\operatorname{degree} \operatorname{deg}(v):=\max \{j \in \mathbb{Z} \mid$ $\left.v \in F^{j} V\right\}$. If both $V$ and $W$ are filtered vector spaces, then their tensor product $V \otimes W$ is filtered with $F^{j}(V \otimes W)=\operatorname{span}\{v \otimes w \mid v \in V, w \in W, \operatorname{deg}(v)+\operatorname{deg}(w) \geq j\}$. The underlying field is considered filtered with $F^{0} k=k$ and $F^{1} k=\{0\}$. A linear map $f: V \rightarrow W$ is said to be filtered of degree $\ell \in Z$ if $f\left(F^{j} V\right) \subseteq F^{j+\ell} W$ for all $j \in \mathbb{Z}$. We write $\operatorname{deg}(f)=\ell$. Again, for the composition of filtered linear maps $f: V \rightarrow W$ and $g: W \rightarrow U$, we have $\operatorname{deg}(g \circ f)=\operatorname{deg}(g)+\operatorname{deg}(f)$. If $V$ is a filtered vector space, we define for each $j \in \mathbb{Z}$ a filtered vector space $V\{j\}$ by $F^{\ell}(V\{j\}):=F^{\ell-j} V$. If the linear map $f: V \rightarrow W$ is filtered of degree $\ell$, then the map $f: V \rightarrow W\{-\ell\}$ is filtered of degree 0 .

If $V$ is a graded vector space with $V \cong \bigoplus_{j \in \mathbb{Z}} V^{j}$, then it is filtered with $F^{j} V=\bigoplus_{\ell \geq j} V^{\ell}$. If $V$ is a filtered vector space, there is an associated graded vector space $V \cong \bigoplus_{j \in \mathbb{Z}} V^{j}$ with $V^{j}:=F^{j} V / F^{j+1} V$. If $f: V \rightarrow W$ is a filtered map of degree $\ell$ between filtered vector spaces, then $f\left(F^{j} V\right) \subseteq F^{j+\ell} W$ for all $j$, and so $f$ descends to the quotients to give maps $f^{j}: V^{j} \rightarrow W^{j+\ell}$. It thereby yields the associated graded map $\bar{f}: V \rightarrow W$ which is graded of degree $\ell$. If $f: V \rightarrow W$ and $g: W \rightarrow U$ are filtered maps of some degree with associated graded maps $\bar{f}$ and $\bar{g}$, then $\bar{g} \circ \bar{f}$ is the associated graded map of $g \circ f$.

The category grdVect gr $_{k}$ graded $k$-vector spaces has graded $k$-vector spaces as objects and graded $k$-linear maps of degree 0 as morphisms. The category fltVect $k$ of filtered $k$-vector spaces has $k$-vector spaces with bounded filtration as objects and filtered $k$-linear maps of degree 0 as morphisms. Both categories are abelian symmetric monoidal categories.

Definition 2.13. An open-closed TQFT $Z: \mathbf{2} \mathbf{C o b}^{\text {ext }} \rightarrow$ Vect $_{k}$ is called Euler-graded [Eulerfiltered] if the vector spaces $C:=Z((0))$ and $A:=Z((1))$ are both graded [filtered] and if for every morphism $M$ of $\mathbf{2} \mathbf{C o b}^{\text {ext }}$, the associated linear map $Z(M)$ is graded [filtered] of degree $\operatorname{deg}(Z(M))=d(M)$ where

$$
d(M):=2 \chi(M)-\left|\Pi_{0}\left(\partial_{1} M\right)\right|+\omega(M)
$$

i.e. twice the Euler characteristic minus the number of connected components of the coloured boundary $\partial_{1} M$ of $M$ plus the window number $\omega(M)$ of $M$. The window number $\omega(M)$ is the number of components of $\partial_{1} M$ that are diffeomorphic to $S^{1}$.

Proposition 2.14. The degree $d(M)$ of (2.37) has the following properties.

1. It forms a topological invariant, and so it is well defined for the morphisms of $\mathbf{2} \mathbf{C o b}^{\text {ext }}$ which are equivalence classes of open-closed cobordisms modulo orientation-preserving diffeomorphisms that restrict to the identity on the black boundary.

2. If $M$ is a cylinder over an arc or a circle, then $d(M)=0$.

3. If $M=M_{1} \otimes M_{2}$ is a tensor product (disjoint union) of open-closed cobordisms, then $d(M)=d\left(M_{1}\right)+d\left(M_{2}\right)$.

4. If $M=M_{1} \circ M_{2}$ is a composition of open-closed cobordisms, then $d(M)=d\left(M_{1}\right)+d\left(M_{2}\right)$.

Proof.

1. This is obvious.

2. In order to prove (2.) to (4.), it is easiest to consider a simplicial model for $M$. For (2.), one can read off the degree from equations (4.10) and (4.11) of [17]. 
3. This is obvious.

4. First, gluing two open-closed cobordisms along a circle has the following effect. For simplicity suppose that as a cell complex the circle is constructed from a single vertex and a single edge. Then gluing along this circle reduces the number of vertices by one and the number of edges by one, and so it leaves the Euler characteristic unchanged. Second, gluing two open-closed cobordisms along an arc with the simplest cell structure has the following effect. It reduces the number of vertices by two and the number of edges by one, and so it reduces the Euler characteristic by one. The claim holds because at the same time the gluing reduces $\left|\Pi_{0}\left(\partial_{1} M\right)\right|-\omega(M)$ by two. If two pairs of disjoint arcs of $\partial_{1} M$ are joined by the gluing, $\left|\Pi_{0}\left(\partial_{1} M\right)\right|$ is reduced by two. If the gluing does not join two disjoint arcs, but rather the two ends of a single arc of $\partial_{1} M$, then $\Pi_{0}\left(\partial_{1} M\right)$ is unchanged, but $\omega(M)$ increases.

Definition 2.15. A knowledgeable Frobenius algebra $\left(A, C, \imath, \imath^{*}\right)$ is called Euler-graded [Eulerfiltered] if both $A$ and $C$ are graded [filtered] vector spaces and if the structure maps are graded [filtered] of the following degrees:

$$
\begin{array}{rlrlrl}
\operatorname{deg}\left(\mu_{A}\right)=-1, & \operatorname{deg}\left(\eta_{A}\right) & =1, & \operatorname{deg}\left(\Delta_{A}\right) & =-1, & \operatorname{deg}\left(\varepsilon_{A}\right)=1, \\
\operatorname{deg}\left(\mu_{C}\right)=-2, & \operatorname{deg}\left(\eta_{C}\right) & =2, & \operatorname{deg}\left(\Delta_{C}\right) & =-2, & \operatorname{deg}\left(\varepsilon_{C}\right)=2, \\
\operatorname{deg}(\imath) & =-1, & \operatorname{deg}\left(\imath^{*}\right) & =-1 . &
\end{array}
$$

Proposition 2.16. An open-closed TQFT $Z: \mathbf{2 C o b}^{\text {ext }} \rightarrow$ Vect $_{k}$ is Euler-graded [Euler-filtered] if and only if its associated knowledgeable Frobenius algebra is Euler-graded [Euler-filtered].

Proof. Given an Euler-graded [Euler-filtered] open-closed TQFT Z, one simply computes (2.37) for the generators of (1.12) in order to obtain (2.38). Triangulations of the generators are displayed, for example, in equation (4.15) of [17].

Let now $\left(A, C, \imath, \imath^{*}\right)$ be an Euler-graded [Euler-filtered] knowledgeable Frobenius algebra and $Z$ be an open-closed TQFT whose associated knowledgeable Frobenius algebra is given by $\left(A, C, \imath, \imath^{*}\right)$. Every open-closed cobordism $M$ is equivalent to a composition and tensor product of the generators (1.12) (Proposition 3.9 of [15]). The degree is defined in (2.38) in such a way that the claim (2.37) holds for each generator. By Proposition 2.14 it holds for tensor products with other generators, for tensor products with cylinders, and for composites of these. This implies that $\operatorname{deg}(Z(M))=d(M)$ as in (2.37) for all morphisms $M$.

\section{Remark 2.17.}

1. In (2.37), we employ twice the degree used by Bar-Natan [5] in order to have integer degrees for all open-closed cobordisms. This means in particular that on $C$, our degrees are twice Khovanov's [1] and that the Jones polynomial arises in our case as a polynomial in an indeterminate $A$ with $q=A^{2}$ rather than in terms of $q$ itself.

2. The right hand side of (2.37) is also additive under gluing open-closed cobordisms along a component of their coloured boundary.

3. A TQFT $Z: \mathbf{2 C o b} \rightarrow$ Vect $_{k}$ is Euler-graded [Euler-filtered] if and only if its associated commutative Frobenius algebra $C=Z(1)$ is Euler-graded [Euler-filtered], i.e. satisfies the conditions of (2.38) for $\mu_{C}, \eta_{C}, \Delta_{C}$, and $\varepsilon_{C}$.

Example 2.18. The commutative Frobenius algebra $C_{0,0}$ of Khovanov (Definition 1.1) is Eulergraded with $\operatorname{deg}(1)=2$ and $\operatorname{deg}(x)=-2$. This grading is uniquely determined by the conditions $\operatorname{deg}\left(\mu_{C}\right)=-2$ and $\operatorname{deg}\left(\Delta_{C}\right)=-2$, exploiting that $\mu_{C}(1 \otimes 1)=1$ and $\Delta_{C}(x)=x \otimes x$. For arbitrary $h, t \in k$, the commutative Frobenius algebra $C_{h, t}$ is Euler-filtered with $\operatorname{deg}_{C}(1)=2$ and $\operatorname{deg}_{C}(x)=-2$. 


\subsection{Commutative and anti-commutative cubes}

In this and in the next section, we recall the definition of the commutative and anti-commutative cubes of [1]. We write this section for commutative cubes in arbitrary abelian monoidal categories $\mathcal{C}$, but for the remainder of this article, it suffices if the reader specializes everything to the

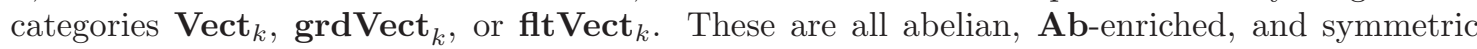
monoidal (see, for example [19]).

Given a finite set $\mathcal{I}$, we denote by $r(\mathcal{I}):=\{(\alpha, j) \in \mathcal{P}(\mathcal{I}) \times \mathcal{I} \mid j \notin \alpha\}$ the set of all pairs $(\alpha, j)$ where $\alpha \subseteq \mathcal{I}$ is a subset that does not contain $j \in \mathcal{I}$. We denote by $\sqcup$ the disjoint union of sets.

Definition 2.19. For a category $\mathcal{C}$ and a finite set $\mathcal{I}$, an $\mathcal{I}$-cube $X=\left(\left\{X_{\alpha}\right\}_{\alpha \subseteq \mathcal{I}},\left\{X_{(\alpha, j)}\right\}_{(\alpha, j) \in r(\mathcal{I})}\right)$ in $\mathcal{C}$ consists of a family of objects $X_{\alpha}$ and of a family of morphisms $X_{(\alpha, j)}: X_{\alpha} \rightarrow X_{\alpha \sqcup\{j\}}$ of $\mathcal{C}$. The objects $X_{\alpha}$ are called the vertices of the cube, and the morphisms $X_{(\alpha, j)}$ the edges. For a given vertex $X_{\alpha}$, we call $|\alpha| \in\{0, \ldots,|\mathcal{I}|\}$ its height.

1. The cube $X$ is called commutative if for all $j, \ell \in \mathcal{I}, j \neq \ell$, and for all $\alpha \subseteq \mathcal{I} \backslash\{j, \ell\}$, the diagram

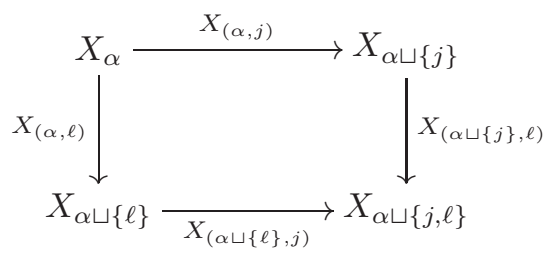

commutes.

2. If $\mathcal{C}$ is an $\mathbf{A b}$-category, the cube $X$ is called anti-commutative if for all $j, \ell \in \mathcal{I}, j \neq \ell$, and for all $\alpha \subseteq \mathcal{I} \backslash\{j, \ell\}$, the above diagram anti-commutes, i.e.

$$
X_{(\alpha \sqcup\{\ell\}, j)} \circ X_{(\alpha, \ell)}=-X_{(\alpha \sqcup\{j\}, \ell)} \circ X_{(\alpha, j)} .
$$

Definition 2.20. Let $\mathcal{C}$ be a monoidal category.

1. Given a finite set $\mathcal{I}$ and $\mathcal{I}$-cubes $X=\left(\left\{X_{\alpha}\right\}_{\alpha},\left\{X_{(\alpha, j)}\right\}_{(\alpha, j)}\right)$ and $Y=\left(\left\{Y_{\alpha}\right\}_{\alpha},\left\{Y_{(\alpha, j)}\right\}_{(\alpha, j)}\right)$, the internal tensor product $X \otimes Y$ is the $\mathcal{I}$-cube with the vertices $(X \otimes Y)_{\alpha}:=X_{\alpha} \otimes Y_{\alpha}$ and with the edges $(X \otimes Y)_{(\alpha, j)}:=X_{(\alpha, j)} \otimes Y_{(\alpha, j)}$.

2. Given finite sets $\mathcal{I}$ and $\mathcal{J}$, an $\mathcal{I}$-cube $X=\left(\left\{X_{\alpha}\right\}_{\alpha \subseteq \mathcal{I}},\left\{X_{(\alpha, j)}\right\}_{(\alpha, j) \in r(\mathcal{I})}\right)$ and a $\mathcal{J}$-cube $Y=\left(\left\{Y_{\beta}\right\}_{\beta \subseteq \mathcal{J}},\left\{Y_{(\beta, \ell)}\right\}_{(\beta, \ell) \in r(\mathcal{J})}\right)$, the external tensor product $X \otimes Y$ is the $\mathcal{I} \sqcup \mathcal{J}$-cube with the vertices $(X \otimes Y)_{\alpha \sqcup \beta}:=X_{\alpha} \otimes Y_{\beta}$ for $\alpha \subseteq \mathcal{I}, \beta \subseteq \mathcal{J}$, and whose edges are defined as follows. If $j \in \mathcal{I} \sqcup \mathcal{J}$, then either $j \in \mathcal{I}$ or $j \in \mathcal{J}$. In the former case, for any $\alpha \subseteq \mathcal{I} \backslash\{j\}$, $\beta \subseteq \mathcal{J}$, we define $(X \otimes Y)_{(\alpha \sqcup \beta, j)}:=X_{(\alpha, j)} \otimes \operatorname{id}_{Y_{\beta}}$. In the latter case, for any $\alpha \subseteq \mathcal{I}$ and $\beta \subseteq \mathcal{J} \backslash\{j\}$, we define $(X \otimes Y)_{(\alpha \sqcup \beta, j)}:=\operatorname{id}_{X_{\alpha}} \otimes Y_{(\beta, j)}$.

Both the internal and the external tensor product of two commutative cubes form commutative cubes, too.

Definition 2.21. Let $\mathcal{C}$ be a monoidal $\mathbf{A b}$-category and $\mathcal{I}$ be a finite set equipped with a linear order ' $\leq$ '. We define the anti-commutative $\mathcal{I}$-cube $E_{\mathcal{I}}=\left(\left\{E_{\alpha}^{\mathcal{I}}\right\}_{\alpha},\left\{E_{(\alpha, j)}^{\mathcal{I}}\right\}_{(\alpha, j)}\right)$, as follows. The vertices are given by the unit object of the monoidal structure, $E_{\alpha}^{\mathcal{I}}:=\mathbb{1}$ for all $\alpha \subseteq \mathcal{I}$. The edges are defined by $E_{(\alpha, j)}^{\mathcal{I}}:=(-1)^{|\{k \in \alpha \mid k<j\}|} \mathrm{id}_{\mathbb{1}}$.

If $\mathcal{C}$ is a monoidal $\mathbf{A b}$-category, $\mathcal{I}$ a finite set, and $X$ a commutative $\mathcal{I}$-cube, then the internal tensor product $X \otimes E_{\mathcal{I}}$ forms an anti-commutative $\mathcal{I}$-cube. 
Definition 2.22. Let $\mathcal{C}$ be a category, $\mathcal{I}$ and $\mathcal{J}$ be finite sets, $X$ be a commutative $\mathcal{I}$-cube and $Y$ be a commutative $\mathcal{J}$-cube. A homomorphism $f: X \rightarrow Y$ of commutative cubes is a pair $f=\left(f^{0},\left\{f_{\alpha}\right\}_{\alpha \subseteq \mathcal{I}}\right)$ consisting of a bijection $f^{0}: \mathcal{I} \rightarrow \mathcal{J}$ and a family of morphisms $f_{\alpha}: X_{\alpha} \rightarrow Y_{f^{0}(\alpha)}$ such that for all $j \in \mathcal{I}$ and $\alpha \subseteq \mathcal{I} \backslash\{j\}$, the diagram

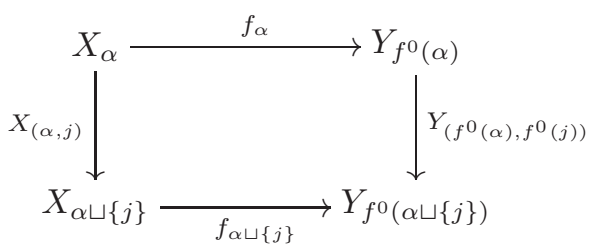

commutes.

The category $\operatorname{Cube}(\mathcal{C})$ of commutative cubes in $\mathcal{C}$ has as objects pairs $(\mathcal{I}, X)$ consisting of a finite set $\mathcal{I}$ equipped with a linear order and a commutative $\mathcal{I}$-cube $X$. The morphisms $f:(\mathcal{I}, X) \rightarrow$ $(\mathcal{J}, Y)$ are homomorphisms $f=\left(f^{0},\left\{f_{\alpha}\right\}_{\alpha}\right)$ of commutative cubes such that for all $j_{1}, j_{2} \in \mathcal{I}$, we have $f^{0}\left(j_{1}\right)<f^{0}\left(j_{2}\right)$ if and only if $j_{1}<j_{2}$. In particular, if $\mathcal{I}$ and $\mathcal{J}$ are not in bijection, then there is no morphism $(\mathcal{I}, X) \rightarrow(\mathcal{J}, Y)$.

If $\mathcal{C}$ is a [symmetric] monoidal category, then so is $\operatorname{Cube}(\mathcal{C})$. The tensor product is given by the external tensor product of cubes, $(\mathcal{I}, X) \otimes(\mathcal{J}, Y):=(\mathcal{I} \sqcup \mathcal{J}, X \otimes Y)$. Here $\mathcal{I} \sqcup \mathcal{J}$ is equipped with the linear order that restricts to the linear order of $\mathcal{I}$ and $\mathcal{J}$, respectively, and for which $j<\ell$ for all $j \in \mathcal{I}$ and $\ell \in \mathcal{J}$. The unit object $\mathbb{1}:=\left(\emptyset,\left\{\mathbb{1}_{\emptyset}\right\}\right)$ is given by the empty set and the commutative $\emptyset$-cube whose only vertex is $\mathbb{1}_{\emptyset}:=\mathbb{1}$. The associativity and unit constraints [and the symmetric braiding] are inherited from $\mathcal{C}$.

\subsection{Complexes}

Definition 2.23. Let $\mathcal{C}$ be an abelian monoidal category.

1. Let $\mathcal{I}$ be a finite set and $X=\left(\left\{X_{\alpha}\right\}_{\alpha},\left\{X_{(\alpha, j)}\right\}_{(\alpha, j)}\right)$ be an anti-commutative $\mathcal{I}$-cube. Then we define a bounded complex $C(X)=\left(C^{i}(X), d^{i}\right)$ as follows. The $i$-th term of this complex is given by

$$
C^{i}(X):=\bigoplus_{\alpha \subseteq \mathcal{I},|\alpha|=i} X_{\alpha},
$$

for $0 \leq i \leq|\mathcal{I}|$, and the differential $d^{i}: C^{i}(X) \rightarrow C^{i+1}(X)$ is given on the $X_{\alpha}, \alpha \subseteq \mathcal{I},|\alpha|=i$, by

$$
\left.d^{i}\right|_{X_{\alpha}}:=\sum_{j \in \mathcal{I} \backslash \alpha} X_{(\alpha, j)}
$$

2. For a pair $(\mathcal{I}, X)$ of a finite set $\mathcal{I}$, equipped with a linear order, and a commutative $\mathcal{I}$-cube $X=\left(\left\{X_{\alpha}\right\}_{\alpha},\left\{X_{(\alpha, j)}\right\}_{(\alpha, j)}\right)$, we define the total complex as

$$
C_{\text {tot }}(\mathcal{I}, X):=C\left(X \otimes E_{\mathcal{I}}\right) .
$$

Proposition 2.24 (Lemma 3 of [14]). Let $\mathcal{C}$ be an abelian monoidal category and $\mathcal{I}, \mathcal{J}$ be finite sets each equipped with a linear order. Let $X$ be a commutative $\mathcal{I}$-cube and $Y$ be a commutative $\mathcal{J}$-cube. Then the following two complexes are isomorphic:

$$
C_{\text {tot }}(\mathcal{I} \sqcup \mathcal{J}, X \otimes Y) \cong C_{\text {tot }}(\mathcal{I}, X) \otimes C_{\text {tot }}(\mathcal{J}, Y) .
$$

Here we choose the linear order on $\mathcal{I} \sqcup \mathcal{J}$ so that it restricts to the linear orders of both $\mathcal{I}$ and $\mathcal{J}$, and so that $j<\ell$ for all $j \in \mathcal{I}$ and $\ell \in \mathcal{J}$. 
Proposition 2.25. Let $\mathcal{C}$ be an abelian monoidal category, $\mathcal{I}$ and $\mathcal{J}$ be finite sets each equipped with a linear order, $X$ be a commutative $\mathcal{I}$-cube and $Y$ be a commutative $\mathcal{J}$-cube. For every homomorphism of commutative cubes

$$
f=\left(f^{0},\left\{f_{\alpha}\right\}_{\alpha}\right):(\mathcal{I}, X) \rightarrow(\mathcal{J}, Y),
$$

there is a morphism of total complexes $C_{\text {tot }}(f): C_{\text {tot }}(\mathcal{I}, X) \rightarrow C_{\text {tot }}(\mathcal{J}, Y)$ whose components $C_{\text {tot }}^{i}(f): C_{\text {tot }}^{i}(\mathcal{I}, X) \rightarrow C_{\text {tot }}^{i}(\mathcal{J}, Y)$ are given on the $X_{\alpha} \otimes \mathbb{1}, \alpha \subseteq \mathcal{I},|\alpha|=i$, by

$$
\left.C_{\text {tot }}^{i}(f)\right|_{X_{\alpha} \otimes \mathbb{1}}:=f_{\alpha} \otimes \operatorname{id}_{\mathbb{1}} .
$$

If $\mathcal{C}$ is an abelian category, we denote by $\operatorname{Kom}(\mathcal{C})$ the category whose objects are complexes in $\mathcal{C}$ and whose morphisms are morphisms of complexes. If $\mathcal{C}$ is [symmetric] monoidal, then so is $\operatorname{Kom}(\mathcal{C}) . \operatorname{Kom}(\mathcal{C})$ is actually a strict 2-category whose 2 -morphisms are homotopies. We denote by $\mathcal{K}(\mathcal{C})$ the decategorification of the 2 -category $\operatorname{Kom}(\mathcal{C})$, i.e. the homotopy category whose objects are the objects of $\operatorname{Kom}(\mathcal{C})$ and whose morphisms are homotopy classes of morphisms of $\operatorname{Kom}(\mathcal{C})$. As usual, we call complexes isomorphic if they are isomorphic in $\operatorname{Kom}(\mathcal{C})$ and homotopy equivalent if they are equivalent in $\operatorname{Kom}(\mathcal{C})$, i.e. isomorphic in $\mathcal{K}(\mathcal{C})$.

The point of Proposition 2.24 and 2.25] is that for an abelian [symmetric] monoidal category $\mathcal{C}$, there is a functor $C_{\text {tot }}$ : $\operatorname{Cube}(\mathcal{C}) \rightarrow \operatorname{Kom}(\mathcal{C})$ sending each commutative cube $(\mathcal{I}, X)$ in $\mathcal{C}$ to its total complex $C_{\text {tot }}(\mathcal{I}, X)$ and each morphism of commutative cubes to the morphism of Proposition 2.25. This functor has a strong monoidal structure $\left(C_{\mathrm{tot}}, C_{\mathrm{tot} 0}, C_{\mathrm{tot}(\mathcal{I}, X),(\mathcal{J}, Y)}\right)$. The isomorphism $C_{\text {tot } 0}: \mathbb{1}_{\mathbf{K o m}(\mathcal{C})} \rightarrow C_{\text {tot }}\left(\mathbb{1}_{\mathbf{C u b e}(\mathcal{C})}\right)$ is the identity morphism on the 1-term complex $\mathbb{1}$, the monoidal unit of $\mathcal{C}$. The natural isomorphisms $C_{\text {tot }(\mathcal{I}, X),(\mathcal{J}, Y)}: C_{\text {tot }}(\mathcal{I}, X) \otimes C_{\text {tot }}(\mathcal{J}, Y) \rightarrow$ $C_{\text {tot }}((\mathcal{I}, X) \otimes(\mathcal{J}, Y))$ are the isomorphisms of 2.45).

A graded [filtered] complex is an object of $\mathbf{K o m}\left(\operatorname{grdVect}_{k}\right)$ [of $\mathbf{K o m}\left(\mathrm{fltVect}_{k}\right)$ ]. If $C=$ $\left(C^{i}, d^{i}\right)$ is a graded [filtered] complex, then we denote by $C\{j\}$ the complex whose terms are $C^{i}\{j\}$ (grading shift) and which has the same differentials. By $C[\ell]$ we denote the complex whose terms are $(C[\ell])^{i}:=C^{i-\ell}$ and whose differentials are $d_{C[\ell]}^{i}:=(-1)^{\ell} d^{i}$. We call the operator $[\ell]$ the cohomological degree shift.

\subsection{Spectral sequences}

It turns out that under suitable conditions, one can compute Khovanov's chain complex and homology from the chain complexes of Lee or of Bar-Natan by exploiting their filtration. This construction uses a spectral sequence. In this section, we summarize the key definitions and fix our notation. We adopt the conventions of [20] for spectral sequences except for the indexing of the associated graded complex of a filtered complex which we describe in detail below.

Let $C=\left(C^{i}, d^{i}\right)$ be a filtered complex, i.e. each $C^{i}$ has a bounded filtration $\left\{F^{k} C^{i}\right\}_{k \in \mathbb{Z}}$, and the $d^{i}: C^{i} \rightarrow C^{i+1}$ are filtered linear maps of degree 0 . The associated graded complex is given by $E_{0}=\left(E_{0}^{i}, d_{0}^{i}\right)$ with $E_{0}^{i}=\bigoplus_{k \in \mathbb{Z}} E_{0}^{k, i}$ where $E_{0}^{k, i}:=F^{k} C^{k+i} / F^{k+1} C^{k+i}$ and $\left.d_{0}^{i}\right|_{E_{0}^{k, i}}:=\left.d^{k+i}\right|_{E_{0}^{k, i}}$. Every $v \in E_{0}^{k, i}$ has a representative of $\operatorname{degree} \operatorname{deg}(v)=k$ in the filtered vector space $C^{k+i}$. Here we have shifted the cohomological degree of $E_{0}^{k, i}$ compared with [20]. Since $d^{i}\left(F^{k} C^{i}\right) \subseteq F^{k} C^{i+1}$ for all $i, k$, the differential descends to the quotient and gives rise to graded linear maps $d_{0}^{i}: E_{0}^{i} \rightarrow E_{0}^{i+1}$ of degree 0 which satisfy $d_{0}^{i+1} \circ d_{0}^{i}=0$.

The bounded filtration on $C$ gives rise to a spectral sequence $\left\{\left(E_{r}, d_{r}\right)\right\}_{r \in \mathbb{N}_{0}}$ of cohomological type whose $E_{0}$-page, $E_{0} \cong \bigoplus_{k, i \in \mathbb{Z}} E_{0}^{k, i}$ coincides with the associated graded complex, indexed as above, and which converges to the associated graded space of the homology of $C$ :

$$
E_{\infty}^{k, i} \cong F^{k} H^{k+i}(C) / F^{k+1} H^{k+i}(C) .
$$

In particular, each differential $d_{r}: E_{r} \rightarrow E_{r}$ is of bidegree $(r, 1-r)$, i.e.

$$
d_{r}\left(E_{r}^{k, i}\right) \subseteq E_{r}^{k+r, i+1-r},
$$


and each page is determined by the homology of the previous page as

$$
E_{r+1}^{k, i} \cong H^{k, i}\left(E_{r}, d_{r}\right):=\operatorname{ker} d_{r}^{k, i} / \operatorname{im} d_{r}^{k-r, i+r-1},
$$

where we have written $d_{r}^{k, i}:=\left.d_{r}\right|_{E_{r}^{k, i}}$. The differentials $d_{r}$ are obtained from the differential of the original filtered complex $C$. Note that due to our shifting of the cohomological degree compared with [20], the step from $E_{0}$ to $E_{1}$ already follows the general pattern (2.50), and that, in our notation, the homology of the associated graded complex is encoded in the $E_{1}$-page as follows:

$$
H^{i}\left(E_{0}\right) \cong \bigoplus_{k \in \mathbb{Z}} E_{1}^{k, i-k}
$$

\section{Tangle homology}

In this section, we take Bar-Natan's 'picture world' construction [5], i.e. chain complexes in a category whose morphisms are formal linear combinations of certain surfaces, and employ an open-closed TQFT in order to translate this into algebra and to turn it into a chain complex of vector spaces. This already yields a generalization of Khovanov homology from links to tangles. Whereas Bar-Natan's surfaces can be glued so as to represent the composition of tangles, the resulting chain complexes of vector spaces are not necessarily equipped with an operation for the composition of tangles. We consider this question in Section 4 below.

\subsection{Examples of knowledgeable Frobenius algebras}

We first present examples of knowledgeable Frobenius algebras and thereby of open-closed TQFTs which extend the commutative Frobenius algebras of Khovanov, Lee, and Bar-Natan.

\subsubsection{Examples with trivial $C$}

We start with some knowledgeable Frobenius algebras $\left(A, C, \imath, \imath^{*}\right)$ whose $C$ is the underlying field equipped with the trivial Frobenius algebra structure. Given a knowledgeable Frobenius algebra $\left(A^{\prime}, C^{\prime}, \imath^{\prime}, \imath^{\prime *}\right)$ we can tensor with one of these $\left(A, C, \imath, \imath^{*}\right)$ to form a knowledgeable Frobenius algebra with isomorphic $C^{\prime} \cong C^{\prime} \otimes k$, but with a more interesting symmetric Frobenius algebra $A \otimes A^{\prime}$.

Example 3.1. Let $k$ be a field, $m \in \mathbb{N}$, and $\left(A, \mu_{A}, \eta_{A}\right)$ be the $m \times m$-matrix algebra $A:=M_{m}(k)$. We write $\left\{e_{p q}\right\}_{1 \leq p, q \leq m}$ for the standard basis for which the multiplication reads $\mu_{A}\left(e_{p q} \otimes e_{r s}\right)=$ $\delta_{q r} e_{p s}$ and the unit $\eta_{A}(1)=\sum_{p=1}^{m} e_{p p}$. The centre is $Z(A) \cong k$. Then $\left(A, \mu_{A}, \eta_{A}, \Delta_{A}, \varepsilon_{A}\right)$ forms a symmetric Frobenius algebra with $\Delta_{A}\left(e_{p q}\right)=\alpha \sum_{r=1}^{m} e_{p r} \otimes e_{r q}$ and $\varepsilon_{A}\left(e_{p q}\right)=\delta_{p q} \alpha^{-1}$ for any $\alpha \in k \backslash\{0\}$. The window element reads $a=\mu_{A} \circ \Delta_{A} \circ \eta_{A}=\alpha m \cdot \eta_{A}$, and so $A$ is strongly separable if and only if the characteristic of $k$ does not divide $m$.

There is a state sum knowledgeable Frobenius algebra $\left(A, C, \imath, \imath^{*}\right)$ with $C=k, \mu_{C}(1 \otimes 1)=1$, $\eta_{C}(1)=1, \Delta_{C}(1)=\alpha^{2} 1 \otimes 1, \varepsilon_{C}(1)=\alpha^{-2}, \imath(1)=\sum_{p=1}^{m} e_{p p}$, and $\imath^{*}\left(e_{p q}\right)=\alpha \delta_{p q}$ constructed as in Proposition 2.7 For $\alpha \in\{-1,1\}$, the Frobenius algebra structure on $C=k$ is trivial.

Example 3.2. Let $k$ be a field. The algebra $A=\mathbb{H}_{k}$ of quaternions, i.e. the free associative unital $k$-algebra generated by $I, J, K$ subject to the relations $I^{2}=-1, J^{2}=-1, K^{2}=-1, I J=K$, $J I=-K, J K=I, K J=-I, K I=J$, and $I K=-J$, forms a symmetric Frobenius algebra $\left(A, \mu_{A}, \eta_{A}, \Delta_{A}, \varepsilon_{A}\right)$ with $\Delta_{A}(1)=\alpha(1 \otimes 1-I \otimes I-J \otimes J-K \otimes K), \Delta_{A}(I)=\alpha(1 \otimes I+I \otimes 1+J \otimes$ $K-K \otimes J), \Delta_{A}(J)=\alpha(1 \otimes J+J \otimes 1+K \otimes I-I \otimes K), \Delta_{A}(K)=\alpha(1 \otimes K+K \otimes 1+I \otimes J-J \otimes I)$, $\varepsilon_{A}(1)=\alpha^{-1}$, and $\varepsilon_{A}(I)=\varepsilon_{A}(J)=\varepsilon_{A}(K)=0$ for any $\alpha \in k \backslash\{0\}$. Its window element is $a=4 \alpha \cdot \eta_{A}$, and so $A$ is strongly separable if and only if char $k \neq 2$.

In this case, there is a state sum knowledgeable Frobenius algebra $\left(A, C, \imath, \imath^{*}\right)$ with $C \cong k$, $\mu_{C}(1 \otimes 1)=1, \eta_{C}(1)=1, \Delta_{C}(1)=4 \alpha^{2} \cdot 1 \otimes 1, \varepsilon_{C}(1)=1 / 4 \alpha^{2}, \imath(1)=1, \imath^{*}(1)=4 \alpha$, and $\imath^{*}(I)=\imath^{*}(J)=\imath^{*}(K)=0$ constructed as in Proposition 2.7 For $\alpha \in\{-1 / 2,1 / 2\}$, the Frobenius structure on $C=k$ is the trivial one. 
Similar examples are available for all strongly separable algebras $A$ that are central over $k$, i.e. $Z(A) \cong k$. In these examples, the state sum knowledgeable Frobenius algebra $\left(A, C, \imath, \imath^{*}\right)$ of Proposition 2.7 has $C \cong k$, and one can choose the window element in such a way that the Frobenius algebra structure on $k$ is the trivial one.

Proposition 3.3. Let $k$ be a field. Every strongly separable and central $k$-algebra is a Brauer algebra.

Proof. Every strongly separable $k$-algebra $A$ is finite-dimensional over $k$ and semi-simple. By Wedderburn's theorem, $A$ is a direct product of $m_{j} \times m_{j}$-matrix algebras, $A \cong \bigoplus_{j=1}^{n} M_{m_{j}}\left(L_{j}\right)$, $n \in \mathbb{N}$, whose coefficients are in finite-dimensional skew field extensions $L_{j} / k$. Its centre is $Z(A) \cong \bigoplus_{j=1}^{n} Z\left(L_{j}\right)$. If $A$ is central over $k$, then $n=1$ and $Z\left(L_{1}\right) \cong k$. In particular, $A$ is finite-dimensional, simple, and central and therefore a Brauer algebra.

Example 3.2 now fits into the following general framework.

Proposition 3.4. Let $k$ be a field and $L / k$ be a finite-dimensional skew field extension such that $L$ is a strongly separable and central $k$-algebra. We denote by $g_{\text {can }}: L \otimes L \rightarrow k$ the canonical bilinear form of $L$ and choose an orthogonal $k$-basis $\left(e_{j}\right)_{j}$ for $L$, i.e.

$$
g_{\mathrm{can}}\left(e_{j} \otimes e_{\ell}\right)=\delta_{j \ell} \beta_{j}
$$

for some $\beta_{j} \in k \backslash\{0\}$. In particular, we can choose $e_{1}:=\eta_{L}(1)$ to be the unit of $L$, and so $\beta_{1}=\operatorname{dim}_{k} L$ and because of strong separability the characteristic of $k$ does not divide $\operatorname{dim}_{k} L$. The symmetric Frobenius algebra structures $\left(L, \mu_{L}, \eta_{L}, \Delta_{L}, \varepsilon_{L}\right)$ are parameterized by their window elements which are just scalars $\zeta \in k \backslash\{0\}$ because of centrality. The Frobenius algebra structures then read:

$$
\begin{aligned}
\Delta_{L}\left(e_{j}\right) & =\zeta \sum_{\ell=1}^{\operatorname{dim}_{k} L} \beta_{\ell}^{-1} \mu_{L}\left(e_{j} \otimes e_{\ell}\right) \otimes e_{\ell}, \\
\varepsilon_{L}\left(e_{j}\right) & =\zeta^{-1} \beta_{j} .
\end{aligned}
$$

The state sum knowledgeable Frobenius algebra of Proposition 2.7 is then given by $\left(L, k, \imath, \imath^{*}\right)$ with the following Frobenius algebra structure for $k$ :

$$
\begin{aligned}
\mu_{k}(1 \otimes 1) & =1, \\
\eta_{k}(1) & =1, \\
\Delta_{k}(1) & =\zeta^{2} / \operatorname{dim}_{k} L \cdot 1 \otimes 1, \\
\varepsilon_{k}(1) & =\operatorname{dim}_{k} L / \zeta^{2}
\end{aligned}
$$

and with $\imath(1)=e_{1}, \imath^{*}\left(e_{1}\right)=\zeta$, and $\imath^{*}\left(e_{j}\right)=0$ for all $j \neq 1$. The Frobenius algebra structure of $Z(L) \cong k$ is therefore the trivial one if and only if $\zeta^{2}=\operatorname{dim}_{k} L$.

\subsubsection{Euler-graded examples}

Khovanov's Frobenius algebra $C_{0,0}$ (Definition 1.1) is Euler-graded, and the degrees of its basis vectors $\{1, x\}$ are determined by this requirement (Example 2.18). Let us try to extend Khovanov's Frobenius algebra to an Euler-graded knowledgeable Frobenius algebra.

Proposition 3.5. If $\left(A, C_{0,0}, \imath, \imath^{*}\right)$ is an Euler-graded knowledgeable Frobenius algebra such that $C_{0,0}$ is Khovanov's Frobenius algebra (Definition 1.1), then $A$ is not strongly separable, the underlying field $k$ has finite characteristic, and char $k$ divides $\operatorname{dim}_{k} A$. The value of the disc is,

$$
\varepsilon_{A} \circ \eta_{A}=0,
$$

and the value of the annulus,

$$
\varepsilon_{A} \circ \mu_{A} \circ \Delta_{A} \circ \varepsilon_{A}=0 .
$$


Proof. Since $\mu_{A}\left(\eta_{A}(1) \otimes \eta_{A}(1)\right)=\eta_{A}(1)$, we have $\operatorname{deg}_{A}\left(\eta_{A}(1)\right)=1$ and therefore $\operatorname{deg}_{C}\left(\imath^{*}\left(\eta_{A}(1)\right)\right)=$ 0 . But the basis vectors $\{1, x\}$ of $C_{0,0}$ have degrees $\operatorname{deg}_{C}(1)=2$ and $\operatorname{deg}_{C}(x)=-2$, and so $\imath^{*} \circ \eta_{A}=0$. The window element is

$$
a=\mu_{A} \circ \Delta_{A} \circ \eta_{A}=\mu_{A} \circ \tau_{A, A} \circ \Delta_{A} \circ \eta_{A}=\imath \circ \imath^{*} \circ \eta_{A}=0,
$$

and so $A$ is not strongly separable. Finally, the dimension of $A$ as an element of $\operatorname{End}(k)$ is the value of the annulus,

$$
\operatorname{dim}_{k} A=\varepsilon_{A} \circ a=0
$$

and so char $k$ divides $\operatorname{dim}_{k} A$. Recall that $A$ is a rigid object in Vect $_{k}$, and so $A$ is finitedimensional over $k$.

Since $\operatorname{deg}_{A}\left(\eta_{A}(1)\right)=1$, we have $\operatorname{deg}_{k}\left(\varepsilon_{A}\left(\eta_{A}(1)\right)\right)=2$, but all non-zero vectors of $k$ are in degree 0 , and so $\varepsilon_{A} \circ \eta_{A}=0$.

Example 3.6. Let $k$ be a field and $C_{0,0}$ be Khovanov's commutative Frobenius algebra over $k$ (Definition [1.1). Consider the algebra $A:=k[y] /\left(y^{2}\right)$ with the commutative Frobenius algebra structure $\left(A, \mu_{A}, \eta_{A}, \Delta_{A}, \varepsilon_{A}\right)$ with $\Delta_{A}(1)=1 \otimes y+y \otimes 1, \Delta_{A}(y)=y \otimes y, \varepsilon_{A}(1)=0$, and $\varepsilon_{A}(y)=1$. The window element is $a=2 y$ which is a zero divisor, and so $A$ is not strongly separable. If char $k=2$, then $\left(A, C_{0,0}, \imath, \imath^{*}\right)$ forms a knowledgeable Frobenius algebra with $\imath(1)=1, \imath(x)=0$, $\imath^{*}(1)=0$, and $\imath^{*}(y)=x$. It is Euler-graded with $\operatorname{deg}_{A}(1)=1, \operatorname{deg}_{A}(y)=-1, \operatorname{deg}_{C}(1)=2$, and $\operatorname{deg}_{C}(x)=-2$ where the subscripts indicate which vector space we are referring to.

For a field $k$ of characteristic char $k \neq 2$, the above example fails to satisfy the Cardy condition whose left hand side reads $\left(\mu_{A} \circ \tau_{A, A} \circ \Delta_{A}\right)(1)=2 y$ and $\left(\mu_{A} \circ \tau_{A, A} \circ \Delta_{A}\right)(y)=0$ while its right hand side is $\left(\imath \circ \imath^{*}\right)(1)=0$ and $\left(\imath \circ \imath^{*}\right)(y)=0$.

The previous example is the first one of the following series.

Example 3.7. Let $k$ be a field and $C_{0,0}$ be Khovanov's commutative Frobenius algebra over $k$ (Definition 1.1]. The truncated polynomial algebra $A=k[y] /\left(y^{p}\right), p \geq 2$, forms a commutative and therefore symmetric Frobenius algebra $\left(A, \mu_{A}, \eta_{A}, \Delta_{A}, \varepsilon_{A}\right)$ with $\Delta_{A}\left(y^{\ell}\right)=\sum_{j=0}^{p-1-\ell} y^{j+\ell} \otimes y^{p-1-j}$ for all $\ell \in\{0, \ldots, p-1\}, \varepsilon_{A}\left(y^{p-1}\right)=1$, and $\varepsilon_{A}\left(y^{\ell}\right)=0$ for all $\ell \in\{0, \ldots, p-2\}$. The window element is $a=p y^{p-1}$ which is a zero divisor, and so $A$ is not strongly separable.

If char $k=p$, then $\left(A, C_{0,0}, \imath, \imath^{*}\right)$ forms a knowledgeable Frobenius algebra with $\imath(1)=1$, $\imath(x)=0, \imath^{*}\left(y^{p-1}\right)=1$, and $\imath^{*}\left(y^{\ell}\right)=0$ for all $\ell \in\{0, \ldots, p-2\}$.

If this knowledgeable Frobenius algebra is Euler-graded, we have $\operatorname{deg}\left(y^{p-1}\right)-1=-2$ because $\imath^{*}\left(y^{p-1}\right)=x$. But $-1=\operatorname{deg}\left(y^{p-1}\right)=(p-1) \operatorname{deg}(y)-(p-2)$ implies that $\operatorname{deg}(y)=(p-3) /(p-1)$. This is an integer only if $p \in\{2,3\}$. Indeed, in these two cases, the knowledgeable Frobenius algebra is Euler-graded. If $p=2$, it coincides with Example 3.6 and if $p=3$, we have $\operatorname{deg}_{A}(1)=1$, $\operatorname{deg}_{A}(y)=0$, and $\operatorname{deg}_{A}\left(y^{2}\right)=-1$ as well as $\operatorname{deg}_{C}(1)=2$, and $\operatorname{deg}_{C}(x)=-2$.

For a field $k$ of characteristic char $k \neq p$, the above example fails to satisfy the Cardy condition whose left hand side reads $\left(\mu_{A} \circ \tau_{A, A} \circ \Delta_{A}\right)(1)=p y^{p-1}$ and $\left(\mu_{A} \circ \tau_{A, A} \circ \Delta_{A}\right)\left(y^{\ell}\right)=0$ for all $\ell \in\{0, \ldots, p-2\}$ whereas its right hand side is $\left(\iota \circ \imath^{*}\right)\left(y^{\ell}\right)=0$ for all $\ell \in\{0, \ldots, p-1\}$.

The truncated polynomial algebras for $p \geq 4$ do not yield Euler-graded knowledgeable Frobenius algebras. The truncated polynomial algebra for $p=3$ is, however, at the same time the first example of the following series of examples all of which are Euler-graded.

Example 3.8. Let $k$ be a field and $p=2 n+1, n \in \mathbb{N}$. Consider the $p$-dimensional vector space $A$ with basis $\left\{X_{-n}, X_{-n+1}, \ldots, X_{n}\right\}$. It forms a symmetric Frobenius algebra $\left(A, \mu_{A}, \eta_{A}, \Delta_{A}, \varepsilon_{A}\right)$ with $\mu_{A}\left(X_{1} \otimes X_{j}\right)=X_{j}=\mu_{A}\left(X_{j} \otimes X_{1}\right), \mu_{A}\left(X_{j} \otimes X_{-j}\right)=X_{-1}$ for all $-n \leq j \leq n, \eta_{A}(1)=X_{1}$, $\Delta_{A}(1)=\sum_{\ell=-n}^{n} X_{\ell} \otimes X_{-\ell}, \Delta_{A}\left(X_{-1}\right)=X_{-1} \otimes X_{-1}, \Delta_{A}\left(X_{j}\right)=X_{-1} \otimes X_{j}+X_{j} \otimes X_{-1}$ for all $j \notin\{1,-1\}$, and $\varepsilon_{A}\left(X_{-1}\right)=1$. The operations $\mu_{A}, \eta_{A}, \Delta_{A}$, and $\varepsilon_{A}$ are 0 on all other basis vectors. The window element reads $a=p X_{-1}$. It is a zero divisor, and so $A$ is not strongly separable.

If char $k=p$, then there is a knowledgeable Frobenius algebra $\left(A, C_{0,0}, \imath, \imath^{*}\right)$ with $\imath(1)=1$, $\imath(x)=0, \imath^{*}\left(X_{-1}\right)=x$, and $\imath^{*}\left(X_{j}\right)=0$ for all $j \neq-1$. If we set $\operatorname{deg}_{A}\left(X_{i}\right)=i$, then it is Euler graded. 
Again, for a field $k$ with char $k \neq p$, the example fails to satisfy the Cardy condition whose left hand side gives $\left(\mu_{A} \circ \tau_{A, A} \circ \Delta_{A}\right)(1)=p X_{-1}$ and $\left(\mu_{A} \circ \tau_{A, A} \circ \Delta_{A}\right)\left(X_{j}\right)=0$ for all $j \neq 1$ whereas its right hand side is $\left(\imath \circ \imath^{*}\right)\left(X_{j}\right)=0$ for all $j$.

Remark 3.9. For any of these examples $\left(A, C, \imath, \imath^{*}\right)$, we can take the tensor product with a knowledgeable Frobenius algebra $\left(A^{\prime}, C^{\prime}, \imath^{\prime}, \imath^{\prime *}\right)$ of Section 3.1.1 that has $C^{\prime}=k$ with the trivial Frobenius algebra structure. The tensor product will therefore have the same algebra $C \otimes k \cong C$, but a bigger and possibly non-abelian $A \otimes A^{\prime}$. When one takes the tensor product of knowledgeable Frobenius algebras, the window elements are multiplicative; if both $A$ and $A^{\prime}$ are strongly separable, then so is $A \otimes A^{\prime}$; and the entire construction of Proposition 2.7 is compatible with the tensor product. If the algebra $A^{\prime}$ is entirely in degree 0 , then the tensor product is Euler-graded [Euler-filtered] as soon as $\left(A, C, \imath, \imath^{*}\right)$ is.

In Section 3.1.4 below, we present examples that are not just tensor products of a commutative algebra $A$ with some non-commutative central algebra, but whose non-commutativity is genuine. Also note that if one is interested in modules over a commutative ring rather than in vector spaces over a field, Example 3.8 works for any commutative ring $k$ of odd characteristic, not necessarily prime.

\subsubsection{Euler-filtered examples}

Both Lee's and Bar-Natan's Frobenius algebra $C_{0,1}$ and $C_{1,0}$ are Euler-filtered (Example2.18). Lee and Rasmussen $[12,18]$ and Turner $[13]$ have shown that one can start from the chain complexes of Lee or Bar-Natan and then recover the chain complex and homology of Khovanov from a spectral sequence. In this construction, Khovanov's chain complex appears as the associated graded chain complex of Lee's or Bar-Natan's filtered complex. We are therefore interested in examples in which the basis vectors $\{1, x\}$ of $C_{h, t}$ have matching degrees, i.e. $\operatorname{deg}_{C}(1)=2$ and $\operatorname{deg}_{C}(x)=-2$.

Proposition 3.10. If $\left(A, C, \imath, \imath^{*}\right)$ is an Euler-filtered knowledgeable Frobenius algebra such that $C=C_{h, t}$ (Definition 1.1) with the basis vectors in degree $\operatorname{deg}_{C}(1)=2$ and $\operatorname{deg}_{C}(x)=-2$, then either $A$ is not strongly separable or the window element is $a=\zeta \cdot \eta_{A}$ with $\zeta \in k \backslash\{0\}$. In both cases, the underlying field $k$ has finite characteristic and char $k$ divides $\operatorname{dim}_{k} A$. Both the disc and the annulus are zero, i.e. (3.8) and (3.9) hold.

Proof. Since $\mu_{A}\left(\eta_{A}(1) \otimes \eta_{A}(1)\right)=\eta_{A}(1)$, we have $\operatorname{deg}_{A}\left(\eta_{A}(1)\right) \leq 1$. Since $\imath\left(\eta_{C}(1)\right)=\eta_{A}(1)$, we have $1 \leq \operatorname{deg}_{A}\left(\eta_{A}(1)\right)$, and so $\operatorname{deg}_{A}\left(\eta_{A}(1)\right)=1$.

This implies that $0=\operatorname{deg}_{A}\left(\eta_{A}(1)\right)-1 \leq \operatorname{deg}_{C}\left(\imath^{*}\left(\eta_{A}(1)\right)\right)$. Due to the degrees of the basis vectors of $C$, we have either $\imath^{*}\left(\eta_{A}(1)\right)=0$ or $\imath^{*}\left(\eta_{A}(1)\right)=\zeta \cdot \eta_{C}(1)$ with $\zeta \in k \backslash\{0\}$.

In the first case, the window element is $a=0$ and $A$ therefore not strongly separable whereas in the second case, the window element is

$$
a=\imath \circ \imath^{*} \circ \eta_{A}=\zeta \cdot \imath \circ \eta_{C}=\zeta \cdot \eta_{A} .
$$

As $\operatorname{deg}_{A}\left(\eta_{A}(1)\right)=1$, we have $\operatorname{deg}_{k}\left(\varepsilon_{A}\left(\eta_{A}(1)\right)\right)=1+1 \leq 0$ or $\varepsilon_{A}\left(\eta_{A}(1)\right)=0$. Only the latter is possible, and so $\varepsilon_{A} \circ a=\zeta \cdot \varepsilon_{A} \circ \eta_{A}=0$ and both the disc and the annulus are zero. The remainder of the argument is as in Proposition 3.5

Remark 3.11. Notice that because of $\operatorname{dim}_{k} A=0$ in $k$, Proposition 2.15 of [17] does not apply to the algebra $A$ of Proposition 3.10 in the strongly separable case. The algebra $A$ is not special in the technical sense.

Example 3.12. Let $k$ be a field, $h, t \in k$, and $A_{h, t}=k[y] /\left(y^{2}-h y-t\right)$. Then $\left(A_{h, t}, \mu_{A}, \eta_{A}, \Delta_{A}, \varepsilon_{A}\right)$ forms a commutative Frobenius algebra with $\Delta_{A}(1)=1 \otimes y+y \otimes 1-h \cdot 1 \otimes 1, \Delta_{A}(y)=y \otimes y+t \cdot 1 \otimes 1$, $\varepsilon_{A}(1)=0$, and $\varepsilon_{A}(y)=1$.

Its window element is $a=\left(\mu_{A} \circ \Delta_{A} \circ \eta_{A}\right)(1)=2 y-h$ which satisfies $a^{2}=\left(h^{2}+4 t\right) \cdot 1$, and so $A_{h, t}$ is strongly separable if and only if $h^{2}+4 t \neq 0$.

In this case, Proposition 2.7 yields a knowledgeable Frobenius algebra $\left(A_{h, t}, C, \imath, \imath^{*}\right)$ with $C=$ $Z\left(A_{h, t}\right)=A_{h, t}$ equipped with the commutative Frobenius algebra structure $\left(C, \mu_{C}, \eta_{C}, \Delta_{C}, \varepsilon_{C}\right)$ 
with $\mu_{C}(1 \otimes 1)=1, \mu_{C}(1 \otimes y)=y, \mu_{C}(y \otimes 1)=y, \mu_{C}(y \otimes y)=h y+t, \eta_{C}(1)=1, \Delta_{C}(1)=$ $\left(h^{2}+2 t\right) \cdot 1 \otimes 1-h \cdot(1 \otimes y+y \otimes 1)+2 \cdot y \otimes y, \Delta_{C}(y)=-h t \cdot 1 \otimes 1+2 t \cdot(1 \otimes y+y \otimes 1)+h \cdot y \otimes y$, $\varepsilon_{C}(1)=2 /\left(h^{2}+4 t\right)$, and $\varepsilon_{C}(y)=h /\left(h^{2}+4 t\right)$, with $\imath(1)=1, \imath(y)=y, \imath^{*}(1)=2 y-h$, and $\imath^{*}(y)=h y+2 t$.

If char $k=2$ and $h=1$, this $C$ is isomorphic as a Frobenius algebra to $C_{1, t}$ of Definition 1.1 We summarize this as follows.

Example 3.13. Let $k$ be a field of characteristic 2 and $t \in k$. The algebra $A_{1, t}=k[y] /\left(y^{2}-y-t\right)$ forms a strongly separable symmetric Frobenius algebra $\left(A_{1, t}, \mu_{A}, \eta_{A}, \Delta_{A}, \varepsilon_{A}\right)$ with $\Delta_{A}(1)=1 \otimes$ $y+y \otimes 1-1 \otimes 1, \Delta_{A}(y)=y \otimes y+t \cdot 1 \otimes 1, \varepsilon_{A}(1)=0$, and $\varepsilon_{A}(y)=1$. There is a state sum knowledgeable Frobenius algebra $\left(A_{1, t}, C_{1, t}, \imath, \imath^{*}\right)$ with $C_{1, t}$ as in Definition [1.1 and $\imath(1)=1$, $\imath(x)=y, \imath^{*}(1)=1$, and $\imath^{*}(y)=x$. It is Euler-filtered with $\operatorname{deg}_{A}(1)=1, \operatorname{deg}_{A}(y)=-1$, $\operatorname{deg}_{C}(1)=2$, and $\operatorname{deg}_{C}(x)=-2$.

While the above example contains Bar-Natan's case $C_{1,0}$ for $t=0$, the following example contains Lee's case $C_{0,1}$.

Example 3.14. Let $k$ be a field of characteristic 2 and $t \in k$. The algebra $A_{0, t}=k[y] /\left(y^{2}-t\right)$ forms a commutative and therefore symmetric Frobenius algebra $\left(A_{0, t}, \mu_{A}, \eta_{A}, \Delta_{A}, \varepsilon_{A}\right)$ with $\Delta_{A}(1)=$ $1 \otimes y+y \otimes 1, \Delta_{A}(y)=y \otimes y+t \cdot 1 \otimes 1, \varepsilon_{A}(1)=0$, and $\varepsilon_{A}(y)=1$. Its window element is zero, and so $A_{0, t}$ is not strongly separable.

There is a knowledgeable Frobenius algebra $\left(A_{0, t}, C_{0, t^{2}}, \imath, \imath^{*}\right)$ with $C_{0, t^{2}}=k[x] /\left(x^{2}-t^{2}\right)$ as in Definition 1.1] but with $t^{2}$, and $\imath(1)=1, \imath(x)=t, \imath^{*}(1)=0$, and $\imath^{*}(y)=t+x$. It is Euler-filtered with $\operatorname{deg}_{A}(1)=1, \operatorname{deg}_{A}(y)=-1, \operatorname{deg}_{C}(1)=2, \operatorname{and}_{\operatorname{deg}_{C}}(x)=-2$.

For a field of characteristic char $k \neq 2$ in this example, the left hand side of the Cardy condition gives $\left(\mu_{A} \circ \tau_{A, A} \circ \Delta_{A}\right)(1)=2 y$ and $\left(\mu_{A} \circ \tau_{A, A} \circ \Delta_{A}\right)(y)=2 t$ whereas the right hand side reads $\left(\imath \circ \imath^{*}\right)(1)=0$ and $\left(\imath \circ \imath^{*}\right)(y)=2 t$.

Remark 3.15. Consider the knowledgeable Frobenius algebra of Example [3.14 Consider its structure maps $\mu_{A}, \eta_{A}, \Delta_{A}, \varepsilon_{A}, \mu_{C}, \eta_{C}, \Delta_{C}, \varepsilon_{C}, \imath$, and $\imath^{*}$ which are filtered of degree $-1,1,-1,1$, $-2,2,-2,2,-1$, and -1 , respectively. Their associated graded maps which are graded of the same degrees, are precisely the structure maps of the knowledgeable Frobenius algebra of Example 3.6 Similarly, the structure maps of the knowledgeable Frobenius algebra of Example 3.13 have as their associated graded maps the structure maps of that of Example 3.6] as well.

\subsubsection{Strongly separable examples}

We have not yet studied examples without filtration in greater detail except for the strongly separable case which is needed in state sum open-closed TQFTs (Proposition 2.7).

Remark 3.16. Let $k$ be a field and $h, t \in k$. The algebra $C_{h, t}=k[x] / p(x)$ for $p(x)=x^{2}-h x-t$ of Definition [1.1 is a field if and only if $p(x)$ is irreducible in $k[x]$, i.e. if and only if there exist no $\alpha, \beta \in k$ such that $\alpha+\beta=h$ and $\alpha \beta=-t$. Otherwise, $p(x)=(x-\alpha)(x-\beta)$, and $p(x)$ is therefore reducible.

Note that for any field $k$ and $h, t \in k$, neither $C_{h, 0}$ including Bar-Natan's example nor $C_{0, t^{2}}$ including Lee's example are fields. Over $k=\mathbb{F}_{2}$, however, $C_{1,1}$ is a field. Let us first consider the case in which $C_{h, t}$ is not strongly separable, i.e. $h^{2}+4 t=0$.

Proposition 3.17. Let $k$ be a field and $h, t \in k$ such that $h^{2}+4 t=0$, and let $\alpha, \beta \in k$ such that $\alpha+\beta=h$ and $\alpha \beta=-t$, i.e. $C_{h, t}$ is not a field. Then there exists no state sum knowledgeable Frobenius algebra $\left(A, C_{h, t}, \imath, \imath^{*}\right)$.

Proof. Assume there is such a state sum knowledgeable Frobenius algebra. Then $A$ is strongly separable over $k$ and therefore finite-dimensional and semi-simple. By Wedderburn's theorem, $A \cong \bigoplus_{j=1}^{n} M_{m_{j}}\left(L_{j}\right)$ is a direct product of matrix algebras with coefficients in finite-dimensional 
skew field extensions $L_{j} / k$. Its centre is $C_{h, t}=Z(A) \cong \bigoplus_{j=1}^{n} Z\left(L_{j}\right)$. As $C_{h, t}$ is 2-dimensional over $k$, either $n=1$ and $\operatorname{dim} Z\left(L_{1}\right)=2$ or $n=2$ and $Z\left(L_{1}\right) \cong k, Z\left(L_{2}\right) \cong k$.

Since by assumption, $C_{h, t}$ is not a field, the first case $C_{h, t} \cong Z\left(L_{1}\right)$ is ruled out and therefore $A \cong M_{m_{1}}\left(L_{1}\right) \oplus M_{m_{2}}\left(L_{2}\right)$ with $C_{h, t}=Z(A) \cong k \oplus k$. But this algebra is strongly separable which contradicts the assumption that $h^{2}+4 t=0$.

In particular, the above proposition rules out the existence of state sum knowledgeable Frobenius algebras $\left(A, C, \imath, \imath^{*}\right)$ with $C=C_{0,0}$ (Khovanov's example) over any field and with $C=C_{0, t^{2}}$ (including Lee's example) over fields of characteristic 2. The above proposition does not discuss the situation in which $C_{h, t}$ is a field which we have not pursued any further. The following lemma illustrates that this happens only in very special circumstances.

Lemma 3.18. Let $k$ be a field and $h, t \in k$ such that $h^{2}+4 t=0$ and such that $p(x)=x^{2}-h x-t$ is irreducible in $k[x]$ and therefore $C_{h, t}$ a field. Then char $k=2, h=0, k$ is not perfect, and in particular $k$ is not finite.

Proof. Consider the algebraic derivative $p^{\prime}(x)=2 x-h$. If char $k \neq 2$ or $h \neq 0$, then $p^{\prime}(x) \neq 0$ and therefore $p(x)$ is separable. If $k$ is perfect, $p(x)$ is separable anyway. Since $p(x)$ is irreducible by assumption, the field extension $C_{h, t} / k$ is separable and therefore the algebra $C_{h, t}$ separable over $k$. As $C_{h, t}$ is commutative, it is even strongly separable, but this contradicts the assumption that $h^{2}+4 t=0$.

For any field $k$ and $h, t \in k$ such that $h^{2}+4 t=0$, the above lemma implies that if char $k \neq 2$ or $h \neq 0$ or $k$ is perfect or $k$ is finite, then there exist $\alpha, \beta \in k$ such that $\alpha+\beta=h$ and $\alpha \beta=-t$.

Apart from the case which we have not yet studied in greater detail, we expect state sum TQFTs only when we extend a strongly separable commutative Frobenius algebra. We therefore consider the algebra $C_{h, t}$ in the strongly separable case, and again we restrict ourselves to the case in which it is not a field.

Lemma 3.19. Let $k$ be a field, $h, t \in k$, and $C_{h, t}=k[x] /\left(x^{2}-h x-t\right)$ be strongly separable and not a field, i.e. $h^{2}+4 t \neq 0$ and there exist $\alpha, \beta \in k$ such that $\alpha+\beta=h$ and $\alpha \beta=-t$. Then $C_{h, t} \cong k \oplus k$ as a Frobenius algebra, and the orthogonal idempotent basis $\left\{z_{1}, z_{2}\right\}$ of $C_{h, t}$ associated with this decomposition is given by

$$
z_{1}=\frac{x-\alpha}{\beta-\alpha}, \quad z_{2}=\frac{x-\beta}{\alpha-\beta} .
$$

The Frobenius algebra structure of $C_{h, t}$ (Definition 1.1) reads in this basis as follows:

$$
\begin{aligned}
\varepsilon\left(z_{1}\right) & =-1 / c \\
\varepsilon\left(z_{2}\right) & =1 / c \\
\Delta\left(z_{1}\right) & =-c \cdot z_{1} \otimes z_{1}, \\
\Delta\left(z_{2}\right) & =c \cdot z_{2} \otimes z_{2},
\end{aligned}
$$

where $c=\alpha-\beta$. We have $1=z_{1}+z_{2}$ and $x=\beta z_{1}+\alpha z_{2}$.

Proof. We compute $c^{2}=(\alpha-\beta)^{2}=h^{2}+4 t \neq 0$, and so $c \neq 0$. Then $z_{1}^{2}=z_{1}, z_{2}^{2}=z_{2}, z_{1} z_{2}=0$, and the other claims follow from straightforward computations.

Theorem 3.20. Let $k$ be a field, $h, t \in k$, and let $C_{h, t}=k[x] /\left(x^{2}-h x-t\right)$ with the Frobenius algebra structure of Definition 1.1] be strongly separable and not a field.

1. Every state sum knowledgeable Frobenius algebra $\left(A, C_{h, t}, \imath, \imath^{*}\right)$ is of the form

$$
A \cong\left(M_{m_{1}}(k) \otimes L_{1}\right) \oplus\left(M_{m_{2}}(k) \otimes L_{2}\right)
$$


where $L_{1} / k$ and $L_{2} / k$ are finite-dimensional strongly separable and central skew field extensions such that the characteristic of $k$ does not divide $m_{1}, m_{2}, \operatorname{dim}_{k} L_{1}$, nor $\operatorname{dim}_{k} L_{2}$. The window element of $A$ is of the form

$$
a=\xi_{1} \cdot z_{1}+\xi_{2} \cdot z_{2}
$$

for some $\xi_{1}, \xi_{2} \in k \backslash\{0\}$ such that

$$
\xi_{1}^{2}=(\alpha-\beta) d_{1}, \quad \text { and } \quad \xi_{2}^{2}=(\beta-\alpha) d_{2},
$$

where we write $z_{1}=\left(I_{m_{1}} \otimes 1,0\right)$ and $z_{2}=\left(0, I_{m_{2}} \otimes 1\right)$ for the basis vectors of the centre $Z(A)$, and $d_{j}:=m_{j}^{2} \operatorname{dim}_{k} L_{j}=\operatorname{dim}_{k}\left(M_{m_{j}}(k) \otimes L_{j}\right)$.

2. The knowledgeable Frobenius algebra $\left(A, C_{h, t}, \imath, \imath^{*}\right)$ of part (1.) is Euler-filtered if and only if the window element of $A$ is of the form

$$
a=\xi \cdot\left(z_{1}+z_{2}\right)=\xi \cdot \eta_{A}(1)
$$

for some $\xi \in k \backslash\{0\}$ and if the characteristic of $k$ divides $d_{1}+d_{2}$ and if

$$
\xi^{2}=(\alpha-\beta) d_{1}
$$

Proof.

1. If $\left(A, C_{h, t}, \imath, \imath^{*}\right)$ is a state sum knowledgeable Frobenius algebra, then $A$ is strongly separable and therefore finite-dimensional over $k$ and semi-simple. By Wedderburn's theorem, $A$ is of the form $A \cong \bigoplus_{j=1}^{n} M_{m_{j}}\left(L_{j}\right)$ where $m_{j} \in \mathbb{N}$ and $L_{j} / k$ are finite-dimensional skew field extensions. Its centre is $Z(A) \cong \bigoplus_{j=1}^{n} Z\left(L_{j}\right)$. As $C_{h, t}$ is 2 -dimensional over $k$, we have either $n=2$ and $\operatorname{dim}_{k} Z\left(L_{1}\right)=2$ or $n=2$ and $Z\left(L_{1}\right) \cong k \cong Z\left(L_{2}\right)$. Since by assumption, $C_{h, t}$ is not a field, only the second case is possible, and therefore $A$ is of the form (3.18) with skew field extensions $L_{1} / k$ and $L_{2} / k$ that are central. Since $A$ is strongly separable, so are $M_{m_{j}}(k) \otimes L_{j}$ as well as $M_{m_{j}}(k)$ and $L_{j}$, and so the characteristic of $k$ does not divide $m_{1}$, $m_{2}, \operatorname{dim}_{k} L_{1}$, nor $\operatorname{dim}_{j} L_{2}$.

Any symmetric Frobenius algebra structure on the strongly separable $A$ is characterized by its invertible window element $a=\xi_{1} \cdot z_{1}+\xi_{2} \cdot z_{2}$ where $\left\{z_{1}, z_{2}\right\}$ is the orthogonal idempotent basis of the centre $Z(A)$ as specified above. We can now compute the state sum knowledgeable Frobenius algebra $\left(A, C=Z(A), \imath, \imath^{*}\right)$ of Proposition 2.7 for this choice of $A$. This computation makes use of Example 3.1 (don't forget to rescale the window element appropriately) and of Proposition 3.4 If we denote by $\left(e_{p q}\right)_{p, q}$ the standard basis of the matrix algebra $M_{m}(k)$ and by $\left(e_{\ell}\right)_{\ell}$ the basis of the skew field $L$ as in Proposition 3.4 we have the basis vectors $e_{p q}^{(1)} \otimes e_{\ell}^{(1)}:=\left(e_{p q} \otimes e_{\ell}, 0\right), 1 \leq p, q \leq m_{1}, 1 \leq \ell \leq \operatorname{dim}_{k} L_{1}$ and $e_{p q}^{(2)} \otimes e_{\ell}^{(2)}:=\left(0, e_{p q} \otimes e_{\ell}\right), 1 \leq p, q \leq m_{2}, 1 \leq \ell \leq \operatorname{dim}_{k} L_{2}$ for $A$ as in (3.18). The knowledgeable Frobenius algebra structure is then given by

$$
\begin{gathered}
\mu_{A}\left(\left(e_{p q}^{(j)} \otimes e_{\ell}^{(j)}\right) \otimes\left(e_{r s}^{\left(j^{\prime}\right)} \otimes e_{i}^{\left(j^{\prime}\right)}\right)\right)=\delta_{j j^{\prime}} \delta_{q r} e_{p s}^{(j)} \otimes\left(\mu_{L_{j}}\left(e_{\ell} \otimes e_{i}\right)\right)^{(j)} \\
\eta_{A}(1)=\sum_{j=1}^{2} \sum_{p=1}^{m_{j}} e_{p p}^{(j)} \otimes e_{1}^{(j)} \\
\Delta_{A}\left(e_{p q}^{(j)} \otimes e_{\ell}^{(j)}\right)=\frac{\xi_{j}}{m_{j}} \sum_{i=1}^{\operatorname{dim}_{k} L_{j}} \sum_{r=1}^{m_{j}} \beta_{i}^{-1}\left(e_{p r}^{(j)} \otimes\left(\mu_{L_{j}}\left(e_{\ell} \otimes e_{i}\right)\right)^{(j)}\right) \otimes\left(e_{r q}^{(j)} \otimes e_{i}^{(j)}\right) \\
\varepsilon_{A}\left(e_{p q}^{(j)} \otimes e_{\ell}^{(j)}\right)=\frac{m_{j}}{\xi_{j}} \delta_{p q} \beta_{\ell}
\end{gathered}
$$




$$
\begin{aligned}
\mu_{C}\left(z_{j} \otimes z_{j^{\prime}}\right) & =\delta_{j j^{\prime}} z_{j}, \\
\eta_{C}(1) & =z_{1}+z_{2}, \\
\Delta_{C}\left(z_{j}\right) & =\frac{\xi_{j}^{2}}{m_{j}^{2} \operatorname{dim}_{k} L_{j}} z_{j} \otimes z_{j}, \\
\varepsilon_{C}\left(z_{j}\right) & =\frac{m_{j}^{2} \operatorname{dim}_{k} L_{j}}{\xi_{j}^{2}}, \\
\imath\left(z_{j}\right) & =\sum_{p=1}^{m_{j}} e_{p p}^{(j)} \otimes e_{1}^{(j)}, \\
\imath^{*}\left(e_{p q}^{(j)} \otimes e_{\ell}^{(j)}\right) & =\frac{\xi_{j}}{m_{j}} \delta_{p q} \delta_{\ell 1} .
\end{aligned}
$$

We have denoted by $\mu_{L_{j}}: L_{j} \otimes L_{j} \rightarrow L_{j}$ the multiplication of $L_{j}$. The Frobenius algebra structure of the centre $C=Z(A)$ is now supposed to agree with that of $C_{h, t}$. Comparing the coefficients in the orthogonal idempotent basis with Lemma 3.19 we obtain the claim.

2. If $\left(A, C_{h, t}, \imath, \imath^{*}\right)$ is Euler-filtered, Proposition 3.10 implies that the window element is of the form $a=\xi \cdot \eta_{A}(1)$ for some $\xi \in k \backslash\{0\}$, i.e. $\xi_{1}=\xi_{2}=\xi$, because $A$ is strongly separable, and that the characteristic of $k$ divides $\operatorname{dim}_{k} A=d_{1}+d_{2}$. In this case, however, $\xi^{2}=c d_{1}$ already implies $\xi^{2}=-c d_{2}$.

For the converse implication, we assume that the characteristic of $k$ divides $d_{1}+d_{2}$ and that $a=\xi \cdot \eta_{A}(1)$ with $\xi^{2}=c d_{1}$. An Euler-filtration for $\left(A, C_{h, t}, \imath, \imath^{*}\right)$ is now given by the following filtrations for $A$ :

$$
A=A^{-1} \supseteq A^{0}=\operatorname{ker} \varepsilon_{A} \supseteq A^{1}=\operatorname{span}_{k}\left\{\eta_{A}(1)\right\} \supseteq A^{2}=\{0\},
$$

and for $C_{h, t}$ :

$$
C^{-2}=C_{h, t} \supseteq C^{-1}=C^{0}=C^{1}=C^{2}=\operatorname{span}_{k}\left\{\eta_{C}(1)\right\} \supseteq C^{3}=\{0\} .
$$

Since $\left(\varepsilon_{A} \circ \eta_{A}\right)(1)=0$ by Proposition 3.10 the above inclusions hold. We now verify that the structure maps of the knowledgeable Frobenius algebra are filtered of the required degrees (2.38).

(a) $\eta_{A}: k \rightarrow A$ is of degree +1 because $\operatorname{deg}_{k}(1)=0$ and $\eta_{A}(1) \in A^{1}$.

(b) $\mu_{A}: A \otimes A \rightarrow A$ is of degree -1 because $\mu_{A}\left(\eta_{A}(1) \otimes \eta_{A}(1)\right)=\eta_{A}(1) \in A^{2} \subseteq A^{0}$, because $\mu_{A}\left(\eta_{A}(1) \otimes a\right)=a \in A^{j}$ for all $a \in A^{j}$, and because $\mu_{A}(a \otimes b) \in A=A^{-1}$ for all $a, b \in A^{0}$.

(c) $\varepsilon_{A}: A \rightarrow k$ is of degree +1 because $\varepsilon_{A}(a)=0$ for all $a \in A^{1}$ or $a \in A^{0}$ and $\varepsilon_{A}(a) \in$ $k^{0}=k$ for all $a \in A^{-1}$.

(d) In order to see that $\Delta_{A}: A \rightarrow A \otimes A$ is of degree -1 , we consider three cases. First, by a direct computation, we show that

$$
\Delta_{A}\left(\eta_{A}(1)\right) \in A \otimes \operatorname{span}_{k}\left\{\eta_{A}(1)\right\}+\operatorname{span}_{k}\left\{\eta_{A}(1)\right\} \otimes A+\operatorname{ker} \varepsilon_{A} \otimes \operatorname{ker} \varepsilon_{A},
$$

and so $\operatorname{deg}_{A}\left(\Delta_{A}\left(\eta_{A}(1)\right)\right) \geq 0$ as required. Second, $\varepsilon_{A}: A \rightarrow k$ is a homomorphism of coalgebras and therefore $\operatorname{ker} \varepsilon_{A}$ a coideal, i.e.

$$
\Delta_{A}\left(\operatorname{ker} \varepsilon_{A}\right) \subseteq A \otimes \operatorname{ker} \varepsilon_{A}+\operatorname{ker} \varepsilon_{A} \otimes A,
$$

and therefore $\operatorname{deg}_{A}\left(\Delta_{A}\left(\operatorname{ker} \varepsilon_{A}\right)\right) \geq-1$. Third, for any $a \in A, \Delta_{A}(a) \in A \otimes A$ and so $\operatorname{deg}_{A}\left(\Delta_{A}(a)\right) \geq-2$ anyway. 
(e) $\imath: C_{h, t} \rightarrow A$ is of degree -1 because $\imath(1)=\eta_{A}(1) \in A^{1}$ and $\imath(x) \in A \subseteq A^{-3}$.

(f) Finally, in order to see that $\imath^{*}: A \rightarrow C_{h, t}$ is of degree -1 , we consider three cases. First, if $a \in A^{-1}$ then $\imath^{*}(a) \in C_{h, t}=C^{-2}$. Second, if $a \in A^{0}=\operatorname{ker} \varepsilon_{A}$, the duality axiom implies that

$$
\varepsilon_{C}\left(\imath^{*}(a)\right)=\varepsilon_{C}\left(\eta_{C}(1) \cdot \imath^{*}(a)\right)=\varepsilon_{A}(\imath(1) \cdot a)=\varepsilon_{A}(a)=0
$$

and therefore $\imath^{*}(a) \in \operatorname{ker} \varepsilon_{C}$ and so $\imath^{*}(a) \in \operatorname{span}_{k}\left\{\eta_{C}(1)\right\}=C^{2}$. This implies that $\operatorname{deg}_{C}\left(\imath^{*}(a)\right)=2 \geq-1$. Third, if $a \in A^{1}$, then $a \in A^{0}$ and $\operatorname{deg}_{C}\left(\imath^{*}(a)\right)=2 \geq 0$.

Remark 3.21. The above theorem establishes conditions under which there exists an Eulerfiltration. In general, this filtration is not unique as we demonstrate by Example 3.25] below.

Example 3.22. If $k$ is a field whose characteristic $p=$ char $k$ is prime of the form $p=4 n+1$, $n \in \mathbb{N}$, then there exist $m_{1}, m_{2} \in \mathbb{N}$ such that $m_{1}<m_{2}$ and $m_{1}^{2}+m_{2}^{2}=p$. The above theorem then yields an example of an Euler-filtered state sum knowledgeable Frobenius algebra $\left(A, C_{1,0}, \imath, \imath^{*}\right)$, i.e. $h=1, t=0, \alpha=1, \beta=0$, and $c=1$, with $\xi=m_{1}, L_{1}=k$, and $L_{2}=k$. Its associated Euler-graded algebra is a knowledgeable Frobenius algebra whose $C$ is Khovanov's $C_{0,0}$.

Example 3.23. Let $k$ be a field of characteristic 5, and let $A=M_{2}(k) \oplus k$ be equipped with the symmetric Frobenius algebra structure that is characterized by the window element $a=\eta_{A}(1)$. This is a special case of Theorem [3.20 (2.), and the state sum knowledgeable Frobenius algebra associated with $A$ is given by $\left(A, C, \imath, \imath^{*}\right)$ with $C=C_{h, t}$ for $h=1, t=0, \alpha=1, \beta=0$, and $c=1$. The Euler-filtration becomes manifest in the basis $(1, A, B, C, D)$ for $A$ where

$$
\begin{gathered}
1=\left(\left(\begin{array}{ll}
1 & 0 \\
0 & 1
\end{array}\right), 1\right), \quad A=\left(\left(\begin{array}{ll}
0 & 1 \\
0 & 0
\end{array}\right), 0\right), \quad B=\left(\left(\begin{array}{ll}
0 & 0 \\
1 & 0
\end{array}\right), 0\right), \\
C=\left(\left(\begin{array}{cc}
1 & 0 \\
0 & -1
\end{array}\right), 0\right), \quad D=\left(\left(\begin{array}{ll}
0 & 0 \\
0 & 0
\end{array}\right), 1\right) .
\end{gathered}
$$

with $\operatorname{deg}_{A}(1)=1, \operatorname{deg}_{A}(A)=\operatorname{deg}_{A}(B)=\operatorname{deg}_{A}(C)=0$, and $\operatorname{deg}_{A}(D)=-1$. We have the basis $\left\{z_{1}, z_{2}\right\}$ with $z_{1}=1-D, z_{2}=D$ for $C=Z(A)$, and the knowledgeable Frobenius algebra structure given by $\eta_{A}(1)=1, \mu_{A}(1 \otimes 1)=1, \mu_{A}(1 \otimes X)=X$ for $X \in\{A, B, C, D\}, \mu_{A}(C \otimes C)=1-D$, $\mu_{A}(D \otimes D)=D, \mu_{A}(A \otimes B)=(1+C-D) / 2, \mu_{A}(B \otimes A)=(1-C-D) / 2, \mu_{A}(A \otimes C)=-A$, $\mu_{A}(C \otimes A)=A, \mu_{A}(B \otimes C)=B, \mu_{A}(C \otimes B)=-B, \varepsilon_{A}(D)=1, \Delta_{A}(1)=-1 \otimes 1-C \otimes C-(1 \otimes$ $D+D \otimes 1)+(A \otimes B+B \otimes A), \Delta_{A}(A)=-(1 \otimes A+A \otimes 1)+(D \otimes A+A \otimes D)-C \otimes A+A \otimes C$, $\Delta_{A}(B)=-(1 \otimes B+B \otimes 1)+(B \otimes D+D \otimes B)-B \otimes C+C \otimes B, \Delta_{A}(C)=-(1 \otimes C+C \otimes$ $1)+(C \otimes D+D \otimes C)+A \otimes B-B \otimes A, \Delta_{A}(D)=D \otimes D, \imath_{A}(1)=1, \imath(x)=D, \imath^{*}(1)=1$, and $\imath^{*}(D)=x$. The structure maps are zero on all basis vectors not listed here.

Example 3.24. Let $k$ be a field of characteristic 5, and let $A=\mathbb{H}_{k} \oplus k$ where $\mathbb{H}_{k}$ is the quaternion algebra (Example 3.2) over $k$. Equip $A$ with the symmetric Frobenius algebra structure characterized by the window element $a=\eta_{A}(1)$. This is a special case of Theorem [3.20 (2.), and the state sum knowledgeable Frobenius algebra $\left(A, C, \imath, \imath^{*}\right)$ associated with $A$ has $C=C_{h, t}$ with $h=1$, $t=0, \alpha=1, \beta=0$, and $c=1$. Its Euler-filtration becomes obvious in the basis $\{1=(1,1), I=$ $(I, 0), J=(J, 0), K=(K, 0), L=(0,1)\}$ with $\operatorname{deg}_{A}(1)=1, \operatorname{deg}_{A}(I)=\operatorname{deg}_{A}(J)=\operatorname{deg}_{A}(K)=0$, and $\operatorname{deg}_{A}(L)=-1$. The knowledgeable Frobenius algebra structure is given by $\eta_{A}(1)=1$, $\mu_{A}(1 \otimes X)=X=\mu_{A}(X \otimes 1)$ for $X \in\{1, I, J, K, L\}, \mu_{A}(I \otimes I)=\mu_{A}(J \otimes J)=\mu_{A}(K \otimes K)=L-1$, $\mu_{A}(I \otimes J)=-\mu_{A}(J \otimes I)=K, \mu_{A}(J \otimes K)=-\mu_{A}(K \otimes J)=I, \mu_{A}(K \otimes I)-\mu_{A}(I \otimes K)=J$, $\mu_{A}(L \otimes L)=L, \varepsilon_{A}(L)=1, \Delta_{A}(1)=-1 \otimes 1+(L \otimes 1+1 \otimes L)+I \otimes I+J \otimes J+K \otimes K, \Delta_{A}(I)=$ $-(1 \otimes I+I \otimes 1)+(L \otimes I+I \otimes L)+J \otimes K-K \otimes J, \Delta_{A}(J)=-(1 \otimes J+J \otimes 1)+(L \otimes J+J \otimes L)+K \otimes I-I \otimes K$, $\Delta_{A}(K)=-(1 \otimes K+K \otimes 1)+(L \otimes K+K \otimes L)+I \otimes J-J \otimes I, \Delta_{A}(L)=L \otimes L, \imath(1)=1, \imath(x)=L$, $\imath^{*}(1)=1, \imath^{*}(L)=x$. The structure maps are zero on all basis vectors not listed here. 
The following example finally shows that the state sum knowledgeable Frobenius algebra of Example 3.23 can be equipped with an Euler-filtration other than that of Theorem 3.20

Example 3.25. The state sum knowledgeable Frobenius algebra of Example 3.23 is Euler-filtered with $\operatorname{deg}_{A}(1)=1, \operatorname{deg}_{A}(A)=-2, \operatorname{deg}_{A}(B)=2, \operatorname{deg}_{A}(C)=0$, and $\operatorname{deg}_{A}(D)=-1$. With this filtration, the associated Euler-graded knowledgeable Frobenius algebra is the one of Example 3.8 in characteristic $p=5$. In order to see this, we set $X_{-2}:=3 A, X_{-1}:=D, X_{0}:=2 C, X_{1}:=D$, and $X_{2}:=B$.

\subsection{Orienting the smoothings of tangle diagrams}

In this section, we define orientations for the smoothings of every plane diagram of a tangle.

Let $T$ be a plane diagram of a tangle with $n$ crossings, numbered $1, \ldots, n$. Let $D_{\alpha} \subseteq \mathbb{R}^{2}$ for subsets $\alpha \subseteq \mathbf{n}$ denote the plane diagrams associated with the smoothings of all crossings such that $j \in \alpha$ if the $j$-th crossing is resolved by the 1 -smoothing and $j \notin \alpha$ if it is resolved by the 0 -smoothing. Each $D_{\alpha}$ is the disjoint union of a finite number of circles and $\operatorname{arcs}$ in $\mathbb{R}^{2}$.

For each tangle diagram $T$, for example for

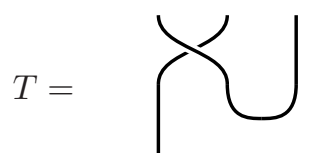

we define two checkerboard colourings. The positive colouring is obtained by shading the region left of the first strand of the tangle and by continuing in a checkerboard fashion. If $T$ is empty, all of $\mathbb{R}^{2}$ is shaded. The negative colouring is obtained from the positive one by replacing shaded with non-shared regions and vice versa. We denote the positively and negatively checkerboard coloured tangle diagrams by $T^{(\varepsilon)}$ for $\varepsilon \in\{+1,-1\}$, respectively. For instance, for the above example,

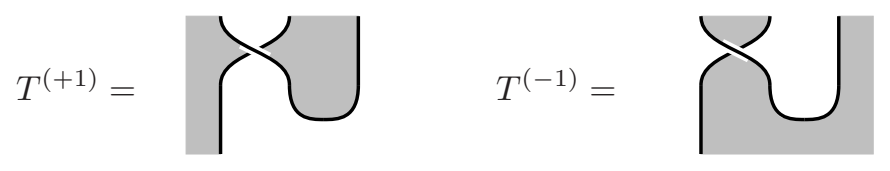

The checkerboard colouring of the tangle diagram $T$ induces a checkerboard colouring on all smoothings $D_{\alpha}, \alpha \subseteq \mathbf{n}$, which we denote by $D_{\alpha}^{(\varepsilon)}$. For the above example $T^{(+1)}$ we have the two smoothings,

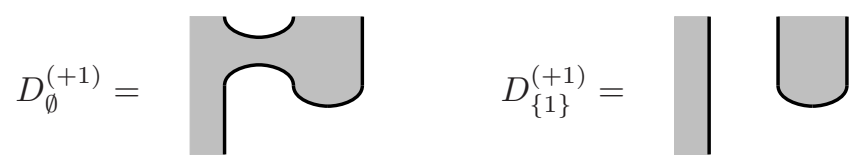

The checkerboard colouring of a smoothing $D_{\alpha}^{(\varepsilon)}$ defines an orientation of the 1-manifold $D_{\alpha}^{(\varepsilon)} \subseteq$ $\mathbb{R}^{2}$. One therefore draws arrows on the components of $D_{\alpha}$ in such a way that the arrows go counter-clockwise around the shaded regions. Often, we omit the shading and indicate only the orientation of the smoothings. For the above examples, we have

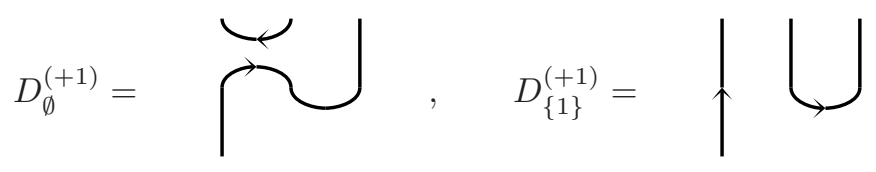

Changing the checkerboard colouring from $\varepsilon$ to $-\varepsilon$ reverses the orientation of the diagram $D_{\alpha}$. Notice that the orientation of the smoothing $D_{\alpha}$ depends only on the checkerboard colouring, but it has nothing to do with a possible orientation of the tangle. 


\subsection{Constructing the tangle chain complex}

In the following definition and theorem, we translate the 2-manifolds with corners used by BarNatan in his 'picture world' construction [5] into the language of our open-closed cobordisms.

Definition 3.26. Let $T$ be a plane diagram of an oriented tangle with $n_{+}$positive crossings ( $ᄌ$ ) and $n_{-}$negative ones $(\lambda), n:=n_{+}+n_{-}$. Number the crossings $1, \ldots, n$. Choose a checkerboard colouring $\varepsilon \in\{-1,+1\}$.

Let $D_{\alpha}^{(\varepsilon)} \subseteq \mathbb{R}^{2}, \alpha \subseteq \mathbf{n}$, denote the plane diagrams associated with the smoothings of all crossings, equipped with the orientation induced from the checkerboard colouring. Number the components of each $D_{\alpha}^{(\varepsilon)}$ by $1, \ldots, k_{\alpha}, k_{\alpha} \in \mathbb{N}_{0}$, and define a sequence $m^{(\alpha)}=\left(m_{1}^{(\alpha)}, \ldots, m_{k_{\alpha}}^{(\alpha)}\right) \in$ $\{0,1\}^{k_{\alpha}}$ such that $m_{\ell}^{(\alpha)}=0$ if the $\ell$-th component is a circle and $m_{\ell}^{(\alpha)}=1$ if it is an arc.

For all $j \in \mathbf{n}$ and $\alpha \subseteq \mathbf{n} \backslash\{j\}$, consider the open-closed cobordism

$$
S_{(\alpha, j)}: D_{\alpha} \rightarrow D_{\alpha \sqcup\{j\}},
$$

which is a cylinder over $D_{\alpha}^{(\varepsilon)}$ except for a sufficiently small neighbourhood of the $j$-th crossing (メ) where it is the saddle

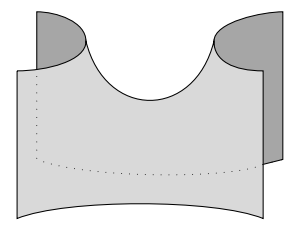

with the 0-smoothing ()() as the source and the 1-smoothing $(\asymp)$ as the target.

We define the $\mathbf{n}$-cube $S_{T, \varepsilon}$ in $\mathbf{2} \mathbf{C o b}^{\text {ext }}$ to have as vertices the sequences $m^{(\alpha)}$ and as edges the (equivalence classes of) the open-closed cobordisms $S_{(\alpha, j)}$.

Remark 3.27. For $j \in \mathbf{n}, \alpha \in \mathbf{n} \backslash\{j\}$, both $D_{\alpha}^{(\varepsilon)}$ and $D_{\alpha \sqcup\{j\}}^{(\varepsilon)}$ are equipped with the orientation induced from the checkerboard colouring $\varepsilon$. This determines the orientation of the open-closed cobordism $S_{(\alpha, j)}$. In order to see this, we display below the relevant saddle which is a smooth oriented 2-manifold with corners $M$ representing an open-closed cobordism, together with its source $D_{\alpha}^{(\varepsilon)}$ and target $D_{\alpha \sqcup\{j\}}^{(\varepsilon)}$ as oriented manifolds.

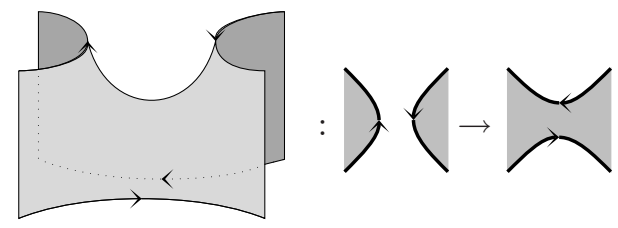

In our pictures, we equip the target of $M$ with the orientation induced from the orientation of $M$ and the source with the opposite of the induced orientation. In this way, the orientation of the smoothing $D_{\alpha}^{(\varepsilon)}$ matches that of the source of $M$ and the orientation of $D_{\alpha \sqcup\{j\}}^{(\varepsilon)}$ matches that of the target.

Theorem 3.28 (see [5]). Given a plane diagram $T$ of an oriented tangle with $n$ crossings and a checkerboard colouring $\varepsilon \in\{-1,+1\}$, the $\mathbf{n}$-cube $S_{T, \varepsilon}$ is commutative.

Proof. The coordinate of the vertical axis of our diagrams (3.44) is a Morse function for the openclosed cobordism. The saddle has a single critical point of index 1 . Let $j, \ell \in \mathbf{n}, j \neq \ell$, and $\alpha \subseteq \mathbf{n} \backslash\{j, \ell\}$. The diagram

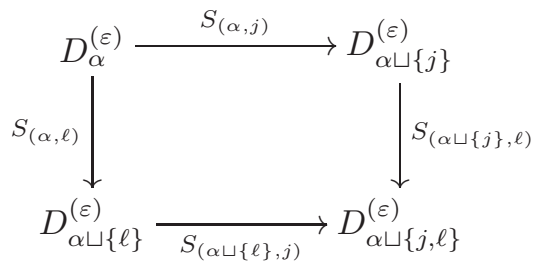


commutes because the two open-closed cobordisms $S_{(\alpha \sqcup\{j\}, \ell)} \circ S_{(\alpha, j)}$ and $S_{(\alpha \sqcup\{\ell\}, j)} \circ S_{(\alpha, \ell)}$ have handle decompositions related by interchanging the critical values of the two index 1 critical points.

Departing from Bar-Natan's work, we now apply an open-closed TQFT $Z: \mathbf{2 C o b}^{\text {ext }} \rightarrow$ Vect $_{k}$ and turn the commutative $\mathbf{n}$-cube $S_{T, \varepsilon}$ in $\mathbf{2} \mathbf{C o b}^{\text {ext }}$ (topology) into a commutative $\mathbf{n}$-cube $Z\left(S_{T, \varepsilon}\right)$ in Vect $_{k}$ (algebra).

Definition 3.29. Let $T$ be a plane diagram of an oriented tangle with $n$ crossings, let $\varepsilon \in\{-1,+1\}$ denote a checkerboard colouring of $T$, and let $Z: \mathbf{2} \mathbf{C o b}^{\text {ext }} \rightarrow$ Vect $_{k}$ be an Euler-graded [Eulerfiltered] open-closed TQFT. We define the $\mathbf{n}$-cube $Z\left(S_{T, \varepsilon}\right)$ in $\operatorname{Vect}_{k}$ to have as vertices the vector spaces $Z\left(m^{(\alpha)}\right)\{2|\alpha|\}$ and as edges the linear maps $Z\left(S_{(\alpha, j)}\right)$.

We use the notation $Z\left(S_{T, \varepsilon}\right)$ because up to a grading shift by $2|\alpha|$ this cube is just the image of $S_{T, \varepsilon}$ under the functor $Z$. We will sometimes just write $S$ and $Z(S)$ rather than $S_{T, \varepsilon}$ and $Z\left(S_{T, \varepsilon}\right)$ if the plane tangle diagram $T$ and the checkerboard colouring $\varepsilon$ are evident from the context.

Proposition 3.30. The $\mathbf{n}$-cube $Z\left(S_{T, \varepsilon}\right)$ is commutative. All edges are graded [filtered] $k$-linear maps of degree 0, and so $Z\left(S_{T, \varepsilon}\right)$ forms an object of Cube $\left(\operatorname{grdVect}_{k}\right)$ [or Cube( fltVect $\left._{k}\right)$ ].

Proof. The cube $Z\left(S_{T, \varepsilon}\right)$ is commutative because $S_{T, \varepsilon}$ is commutative and $Z$ is a functor. Let $M$ be the saddle (3.44) as an open-closed cobordism. It has $\chi(M)=1,\left|\Pi_{0}\left(\partial_{1} M\right)\right|=4$, and $\omega(M)=0$. By Proposition 2.14 and Remark 2.17(2.), we have the degree

$$
\operatorname{deg} S_{(\alpha, j)}=-2
$$

for all $j \in \mathbf{n}, \alpha \subseteq \mathbf{n} \backslash\{j\}$, i.e. for all edges of the cube. The degree shift by twice the height of each vertex $\alpha \subseteq \mathbf{n}$ in $Z\left(m^{(\alpha)}\right)\{2|\alpha|\}$ makes sure that all edges are graded [filtered] of degree 0 .

\section{Remark 3.31.}

1. All the cobordisms $S_{(\alpha, j)}$ have the following property: Each connected component of $\partial_{1} S_{(\alpha, j)}$ has one boundary point belonging to the source and the other to the target of the cobordism. In particular, $S_{(\alpha, j)}$ has window number zero.

2. Inverting the checkerboard colouring does not affect the tensor factors $C$ of the $Z\left(D_{\alpha}\right)$, but it does affect $A$ by replacing it with $A^{\text {op }}$. This is the Frobenius algebra opposite to $A$ whose multiplication and comultiplication are given by $\mu_{A^{\mathrm{op}}}=\mu_{A} \circ \tau_{A, A}$ and $\Delta_{A^{\mathrm{op}}}=\tau_{A, A} \circ \Delta_{A}$.

Remark 3.32. If $T$ is a plane diagram of an oriented link, each 2-dimensional cobordism $S_{(\alpha, j)}$ is the disjoint union of either $?$ or $\boldsymbol{\Omega}$ (pairs of pants) with a finite number of cylinders over the circle.

Now let $T$ be a plane diagram of an oriented tangle with $n$ crossings, $\varepsilon \in\{-1,+1\}$ be a checkerboard colouring and $j \in \mathbf{n}$ denote a crossing. The open-closed cobordism $S_{(\alpha, j)}: D_{\alpha}^{(\varepsilon)} \rightarrow$ $D_{\alpha \sqcup\{j\}}^{(\varepsilon)}$ is the disjoint union of one connected component that contains the saddle with a finite number of cylinders over the circle and over the arc. Depending on the smoothing $\alpha \subseteq \mathbf{n} \backslash\{j\}$ of the other crossings, the component with the saddle can have one of the following forms. On the left, we display the $j$-th crossing with the checkerboard colouring and with the connected components of the diagram $T$ that are attached to that crossing. Then we show the two smoothings $D_{\alpha}^{(\varepsilon)}$ and $D_{\alpha \sqcup\{j\}}^{(\varepsilon)}$ of the $j$-th crossing, and finally on the right we show the associated open-closed cobordism 
as a composition of the generators, read from top to bottom.
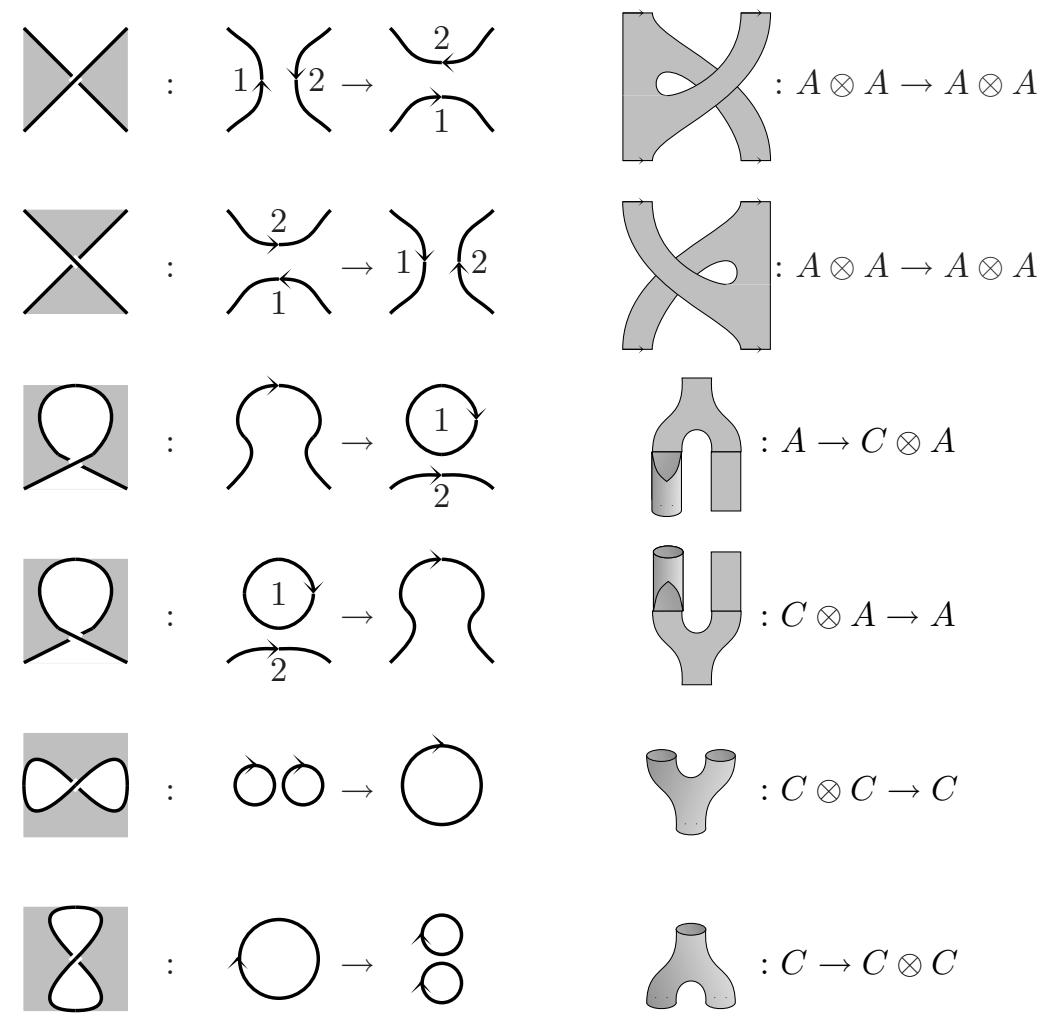

The components of the diagrams $D_{\alpha}^{(\varepsilon)}$ and $D_{\alpha \sqcup\{j\}}^{(\varepsilon)}$ are numbered $1,2, \ldots$ corresponding to the tensor factors in the source and target of the open-closed cobordism on the right. If necessary, we indicate with a little arrow the orientation of the arc in the black boundary of the open-closed cobordism.

All other possibilities for the component of the open-closed cobordism that contains the saddle and the remaining checkerboard colourings can be obtained from these by rotations in the plane. Changing the numbering of the components results in permutations of the tensor factors.

We encourage the reader to visualize how the open-closed cobordism displayed in (3.48) can be obtained from the saddle (3.45) by 'smashing' the saddle flat on the drawing plane and then pulling the source to the top and the target to the bottom of the diagram.

\subsection{The tangle complex is well defined}

In this section, we show that the tangle complex $\llbracket T \rrbracket_{\varepsilon, Z}$ of (3.55) is well defined up to isomorphism regardless of the numbering of the crossings of $T$ and regardless of the numbering of the components of each smoothing $D_{\alpha}^{(\varepsilon)} \subseteq \mathbb{R}^{2}$.

Proposition 3.33. Let $T$ be a plane diagram of an oriented tangle with $n$ crossings, $\varepsilon \in\{-1,+1\}$ be a checkerboard colouring, and let $Z: \mathbf{2} \mathbf{C o b}^{\text {ext }} \rightarrow$ Vect $_{k}$ be an open-closed TQFT. The tangle complex $\llbracket T \rrbracket_{\varepsilon, Z}$ is

1. independent of the numbering of the crossings up to isomorphism.

2. independent of the numbering of components of any smoothing $D_{\alpha}^{(\varepsilon)}$.

Proof. 
1. Given some numbering $1, \ldots, n$ of the crossings of $T$, we represent a change of this numbering by a bijection $f^{0}: \mathbf{n} \rightarrow \mathbf{n}$. Consider the commutative cube $S_{T, \varepsilon}$ of Definition 3.26. There is a homomorphism of commutative cubes $f=\left(f^{0},\left\{f_{\alpha}\right\}_{\alpha \subseteq \mathbf{n}}\right): S_{T, \varepsilon} \rightarrow S_{T, \varepsilon}$ with $f_{\alpha}:=\mathrm{id}_{m^{(\alpha)}}$ whose inverse is given similarly by the inverse bijection $\left(f^{0}\right)^{-1}$.

This isomorphism of commutative cubes yields an isomorphism $Z(f): Z\left(S_{T, \varepsilon}\right) \rightarrow Z\left(S_{T, \varepsilon}\right)$ for the commutative cubes of Definition 3.29] and by Proposition 2.25] an isomorphism of complexes $C_{\text {tot }}(Z(f)): \llbracket T \rrbracket_{\varepsilon, Z} \rightarrow \llbracket T \rrbracket_{\varepsilon, Z}$. Note that permuting the index set $\mathbf{n}$ of the commutative cube induces a permutation on the set of vertices that leaves the height of each vertex fixed.

2. Recall that a linear order of the components of $D_{\alpha}^{(\varepsilon)}$ is needed in order to define the object $m^{(\alpha)}=\left(m_{1}^{(\alpha)}, \ldots, m_{k_{\alpha}}^{(\alpha)}\right) \in\{0,1\}^{k_{\alpha}}$ of $\mathbf{2} \mathbf{C o b}^{\text {ext }}$ associated with $D_{\alpha}^{(\varepsilon)}$ in Definition [3.26]

Let $m^{(\alpha)}$ be this object of $\mathbf{2} \mathbf{C o b}^{\text {ext }}$, and let $\sigma:\left\{1, \ldots, k_{\alpha}\right\} \rightarrow\left\{1, \ldots, k_{\alpha}\right\}$ be a bijection. Then $\sigma$ induces a bijection $\bar{\sigma}:\{0,1\}^{k_{\alpha}} \rightarrow\{0,1\}^{k_{\alpha}}, m^{(\alpha)} \mapsto \sigma\left(m^{(\alpha)}\right):=\left(m_{\sigma^{-1}(1)}^{(\alpha)}, \ldots, m_{\sigma^{-1}\left(k_{\alpha}\right)}^{(\alpha)}\right)$.

Let $S_{T, \varepsilon}$ denote the commutative cube constructed using the ordering of the components $\left(1, \ldots, k_{\alpha}\right)$ for some vertex $\alpha \subseteq \mathbf{n}$, and let $S_{T, \varepsilon}^{\prime}$ denote the commutative cube constructed using the same ordering of components as in $S_{T, \varepsilon}$ except at the vertex $\alpha$, where we use the ordering $\left(\sigma^{-1}(1), \ldots, \sigma^{-1}\left(k_{\alpha}\right)\right)$. Note that the edges of $S_{T, \varepsilon}^{\prime}$ will in general differ from those of $S_{T, \varepsilon}$ due to the different ordering of the components.

There is a homomorphism $f=\left(f^{0},\left\{f_{\beta}\right\}_{\beta \subseteq \mathbf{n}}\right): S_{T, \varepsilon} \rightarrow S_{T, \varepsilon}^{\prime}$ of commutative cubes with $f^{0}=\mathrm{id}_{\mathbf{n}}, f_{\alpha}=\bar{\sigma}$, and $f_{\beta}=\operatorname{id}_{\{0,1\}^{k_{\alpha}}}$ for all $\beta \subseteq \mathbf{n}, \beta \neq \alpha$. Its inverse is given similarly using $\bar{\sigma}^{-1}$ instead of $\bar{\sigma}$.

Just as in part (1.), this isomorphism of commutative cubes induces an isomorphism of tangle complexes.

\section{Remark 3.34.}

1. Part (2.) of the proposition implies in particular that the two saddles (3.48) and (3.49) are related by permutations as follows:

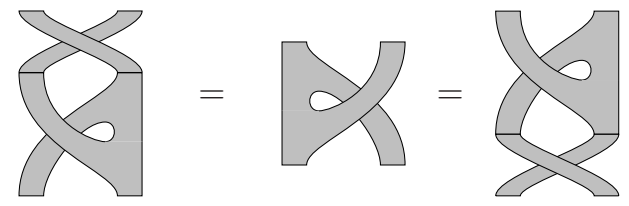

2. Inverting the checkerboard colouring has the effect of systematically replacing $A$ by the opposite Frobenius algebra $A^{\mathrm{op}}$, and of replacing $A^{\mathrm{op}}$-left actions by $A$-right actions and vice versa.

\subsection{Main results}

The following definition and theorem are the main results of this section. They improve BarNatan's result of [5] and yield for every tangle (not just for every link) a complex of vector spaces that is invariant under Reidemeister moves up to homotopy equivalence.

Definition 3.35. Let $T$ be a plane diagram of an oriented tangle with $n_{+}$positive and $n_{-}$ negative crossings, $n:=n_{+}+n_{-}$, let $\varepsilon \in\{-1,+1\}$ denote a checkerboard colouring of $T$, and let $Z: \mathbf{2 C o b}^{\text {ext }} \rightarrow$ Vect $_{k}$ be an Euler-graded [Euler-filtered] open-closed TQFT. We define the tangle complex as the total complex

$$
\llbracket T \rrbracket_{\varepsilon, Z}:=C_{\text {tot }}\left(\mathbf{n}, Z\left(S_{T, \varepsilon}\right)\right)\left\{2 n_{+}-4 n_{-}\right\}\left[-n_{-}\right] .
$$

We will often denote the complex $\llbracket T \rrbracket_{\varepsilon, Z}$ as $\llbracket T \rrbracket$ when no confusion is likely to arise. 
Proposition 3.36. $\llbracket T \rrbracket_{\varepsilon, Z}$ is an object of $\operatorname{Kom}\left(\operatorname{grdVect}_{k}\right)$ or of $\operatorname{Kom}\left(\right.$ flt Vect $\left._{k}\right)$, respectively.

Proof. By Proposition 3.30

Theorem 3.37. Let $T, T^{\prime}$ be plane diagrams of oriented tangles, $\varepsilon \in\{-1,+1\}$, and $Z: \mathbf{2} \mathbf{C o b}^{\text {ext }} \rightarrow$ Vect $_{k}$ be an Euler-graded [Euler-filtered] open-closed TQFT that satisfies Bar-Natan's conditions. If $T$ and $T^{\prime}$ are related by a Reidemeister move or by a plane isotopy, then the complexes $\llbracket T \rrbracket_{\varepsilon, Z}$ and $\llbracket T^{\prime} \rrbracket_{\varepsilon, Z}$ are homotopy equivalent as graded [filtered] complexes.

Proof. The proof is almost the same as Bar-Natan's [5], except that whenever Bar-Natan writes down a 2-manifold with corners, we apply the open-closed TQFT and turn it into a linear map between vector space. We do not reproduce the proof here. We do, however, present the proofs for the invariance under Reidemeister moves up to homotopy equivalence when we develop the composition operation for tangles in Section 4 below.

\section{Remark 3.38.}

1. While the commutative cube $Z\left(S_{T, \varepsilon}\right)$ did not depend on the orientation of the tangle, the tangle complex $\llbracket T \rrbracket_{\varepsilon, Z}$ does depend on it via the grading shift by $2 n_{+}-4 n_{-}$and the homological degree shift by $-n_{-}$. These shifts correspond to the prefactor $(-1)^{n_{-}} A^{2 n_{+}-4 n_{-}}$, $q=A^{2}$, which turns the Kauffman bracket into an invariant under ambient isotopy, and they are needed to prove Theorem 3.37

2. Since we have been able to translate from topology to algebra right in the beginning and since grdVect $_{k}$ and fltVect ${ }_{k}$ are abelian categories, there is no need to consider formal linear combinations of surfaces as in [5].

3. In order to obtain the case in which there is no filtration, one can simply define the grading shift to be the identity, use the trivial filtration $V=F^{0} V \supseteq F^{1} V=\{0\}$ for all vector spaces $V$, and omit the assumption of an Euler-filtration for the TQFT.

Denote by $\mathcal{H}^{r}(C)$ the $r$-th cohomology of the tangle complex $C:=\llbracket T \rrbracket_{\varepsilon, Z}$. If $Z$ is an Eulerfiltered open-closed TQFT, then the filtration $F^{*}$ on the complex $C$ induces a filtration on each homology group given by the image of the inclusion

$$
F^{k} \mathcal{H}^{r}(C):=\operatorname{image}\left(\mathcal{H}^{r}\left(F^{k} C\right) \hookrightarrow \mathcal{H}^{r}(C)\right)
$$

where $F^{k} C:=\bigoplus_{r \in \mathbb{Z}} F^{k} C^{r}$. The filtration defines the following bigrading on homology:

$$
\mathcal{H}^{k, r}(C):=F^{k} \mathcal{H}^{r}(C) / F^{k+1} \mathcal{H}^{r}(C) .
$$

Corollary 3.39. If $Z$ is an Euler-filtered open-closed TQFT that satisfies Bar-Natan's conditions, then the 2-variable tangle polynomial $\mathcal{P}(T)_{\varepsilon, Z} \in \mathbb{Z}\left[t, t^{-1}, A, A^{-1}\right]$ defined as the filtered Poincaré polynomial of the complex $C:=\llbracket T \rrbracket_{\varepsilon, Z}$, i.e.

$$
\mathcal{P}(T)_{\varepsilon, Z}:=\sum_{r, k \in \mathbb{Z}} t^{r} A^{k} \mathrm{rk} \mathcal{H}^{k, r}(C),
$$

is an invariant of the tangle $T$.

Proof. This follows from Theorem 3.37

Proposition 3.40. Let $T$ be a plane diagram of an oriented $(p, q)$-tangle, i.e. its source and its target are a disjoint union of $p$ and $q$ points, respectively. We define $r \in \mathbb{N}_{0}$ in such a way that $2 r=p+q$. Let $\varepsilon \in\{-1,+1\}$ denote a checkerboard colouring and let $Z: \mathbf{2} \mathbf{C o b}^{\text {ext }} \rightarrow$ Vect $_{k}$ be an open-closed TQFT with associated knowledgeable Frobenius algebra $\left(A, C, \imath, \imath^{*}\right)$. Then the tangle complex $\llbracket T \rrbracket_{\varepsilon, Z}$ is a complex of $\left(A^{\otimes r}, A^{\otimes r}\right)$-bimodules. 
Proof. Let $T$ have $n$ crossings. We show that the vertices of the $\mathbf{n}$-cube $Z\left(S_{T, \varepsilon}\right)$ of Definition 3.29 are $\left(A^{\otimes r}, A^{\otimes r}\right)$-bimodules and that its edges are bimodule morphisms. The definition of the total complex which is used in (3.55) then implies that the terms of $\llbracket T \rrbracket_{\varepsilon, Z}$ are $\left(A^{\otimes r}, A^{\otimes r}\right)$-bimodules and that the differential consists of bimodule morphisms.

We use the notation of Definition 3.26. The vertex $\alpha \subseteq \mathbf{n}$ of $Z\left(S_{T, \varepsilon}\right)$ is the vector space

$$
Z\left(m^{(\alpha)}\right)=A^{\left(m_{1}^{(\alpha)}\right)} \otimes \cdots \otimes A^{\left(m_{k_{\alpha}}^{(\alpha)}\right)},
$$

where $A^{(0)}:=C$ and $A^{(1)}:=A$. Obviously $r=\left|\left\{j \in\left\{1, \ldots, k_{\alpha}\right\} \mid m_{j}^{(\alpha)}=1\right\}\right|$, i.e. for every tensor factor of $Z\left(m^{(\alpha)}\right)$ that is an $A$ as opposed to a $C$, the tangle has two boundary points.

By the checkerboard colouring, $r$ out of the $p+q$ boundary points are '-'-boundaries of the oriented smoothing $D_{\alpha}^{(\varepsilon)} \subseteq \mathbb{R}^{2}$, i.e. they coincide with the tail of an arrow in the example (3.42). The other $r$ boundary points are '+'-boundaries and coincide with the head of an arrow. Note that this partitioning of the boundary points of $T$ into '-'- versus ' + '-boundaries depends only on the checkerboard colouring, but that it is independent of the vertex $\alpha \subseteq \mathbf{n}$.

We number the '-'-boundary points by $1, \ldots, r$. For each diagram $D_{\alpha}^{(\varepsilon)}, \alpha \subseteq \mathbf{n}$, the boundary point number $j \in\{1, \ldots, r\}$ is contained in an arc that forms a component of $D_{\alpha}^{(\varepsilon)}$. We denote by $s_{j}^{(\alpha, \varepsilon)} \in\left\{1, \ldots, k_{\alpha}\right\}$ the number corresponding to the tensor factor of this component. We have in particular $m_{s_{j}^{(\alpha, \varepsilon)}}^{(\alpha)}=1$. Similarly, we number the '+'-boundaries by $1, \ldots, r$. For each $\alpha \subseteq \mathbf{n}$, the ' + '-boundary number $j \in\{1, \ldots, r\}$ is contained in a component of $D_{\alpha}^{(\varepsilon)}$. We denote its number by $t_{j}^{(\alpha, \varepsilon)} \in\left\{1, \ldots, k_{\alpha}\right\}$.

Then $Z\left(m^{(\alpha)}\right)$ forms an left $A^{\otimes r}$-module where the $j$-th tensor factor of $A^{\otimes r}$ acts by leftmultiplication on the $s_{j}^{(\alpha, \varepsilon)}$-th tensor factor of $Z\left(m^{(\alpha)}\right)$. Similarly, $Z\left(m^{(\alpha)}\right)$ forms a right $A^{\otimes r_{-}}$ module where the $j$-th tensor factor of $A^{\otimes r}$ acts by right-multiplication on the $t_{j}^{(\alpha, \varepsilon)}$-th tensor factor of $Z\left(m^{(\alpha)}\right)$. By associativity of $A, Z\left(m^{(\alpha)}\right)$ forms an $\left(A^{\otimes r}, A^{\otimes r}\right)$-bimodule.

For instance, the crossing of (3.48) has two '+'-boundary points. Say, number 1 at the top left and number 2 at the bottom right of the diagram. We have $\mathbf{n}=1$, and we denote by $D_{\emptyset}^{(\varepsilon)}$ and $D_{\{1\}}^{(\varepsilon)}$ the two smoothings displayed in (3.48). Then we have $t_{1}^{(\emptyset, \varepsilon)}=1, t_{2}^{(\emptyset, \varepsilon)}=2$, and $t_{1}^{(\{1\}, \varepsilon)}=2$, $t_{2}^{(\{1\}, \varepsilon)}=1$.

From the definition of the open-closed cobordisms, see, for example the cobordisms (3.48) to (3.53), it is obvious that the edges $Z\left(S_{(\alpha, \ell)}\right), \ell \in \mathbf{n}, \alpha \subseteq \mathbf{n} \backslash\{\ell\}$, of the cube form morphisms of $\left(A^{\otimes r}, A^{\otimes r}\right.$ )-bimodules. Every '-'-boundary point $j \in\{1, \ldots, r\}$ corresponds to one component of the coloured boundary $\partial_{1} S_{(\alpha, \ell)}$ of the open-closed cobordism. This component intersects both the source and the target of $S_{(\alpha, \ell)}$ in one point (Remark 3.31). It intersects the $s_{j}^{(\alpha, \varepsilon)}$-th component of the source (which is an arc) and the $s_{j}^{(\alpha \sqcup\{\ell\}, \varepsilon)}$-th component of the target (which is an arc, too). The map $Z\left(S_{(\alpha, j)}\right)$ therefore commutes with the $A$-left actions defined above due to the relations that hold in any open-closed TQFT. An analogous result holds for '+'-boundary points if one replaces $s_{j}^{(\alpha, \varepsilon)}$ and $s_{j}^{(\alpha \sqcup\{\ell\}, \varepsilon)}$ by $t_{j}^{(\alpha, \varepsilon)}$ and $t_{j}^{(\alpha \sqcup\{\ell\}, \varepsilon)}$, respectively.

Let us illustrate this result for the example of the crossing of (3.48) and the '+'-boundary point number 1 at the top left of the diagram, the $A$-right action on the $t_{1}^{(\emptyset, \varepsilon)}=1$-st tensor factor of $Z\left(m^{(\emptyset)}\right)=A \otimes A$ is given by

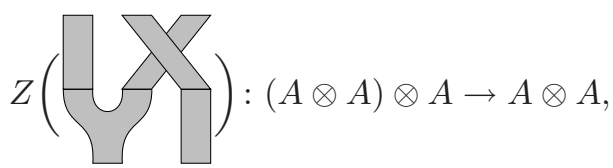

whereas the $A$-right action on the $t_{1}^{(\{1\}, \varepsilon)}=2$-nd tensor factor of $Z\left(m^{(\{1\})}\right)=A \otimes A$ is given by

$$
Z(\square \bigcirc):(A \otimes A) \otimes A \rightarrow A \otimes A .
$$


The $A$-right action commutes with the differential of the complex because of the following relation in $2 \mathrm{Cob}^{\text {ext }}$ :

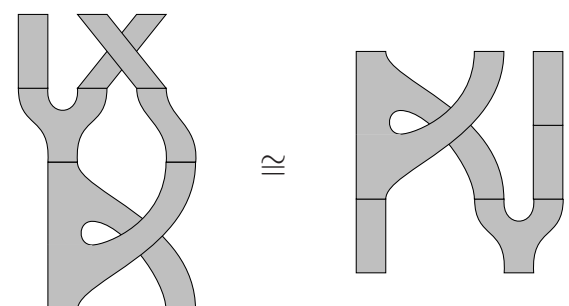

One can literally slide the $A$-right action down along the component of $\partial_{1} M$ that corresponds to the '+'-boundary number 1 . This is the component that connects the right boundary point of the first component of the source with the right boundary point of the second component of the target of the open-closed cobordism of (3.48).

Remark 3.41. For a $(p, q)$-tangle, it is more natural to have $p$ copies of $A$ act from the left and $q$ copies from the right. This can be achieved by replacing $A$-right actions by $A^{\mathrm{op}}$-left actions and vice versa.

We define for $p \in \mathbb{N}_{0}$,

$$
A^{(p,+1)}:=\underbrace{A \otimes A^{\mathrm{op}} \otimes A \otimes A^{\mathrm{op}} \otimes \cdots}_{p}
$$

and

$$
A^{(p,-1)}:=\underbrace{A^{\mathrm{op}} \otimes A \otimes A^{\mathrm{op}} \otimes A \otimes \cdots}_{p} .
$$

Then the tangle complex $\llbracket T \rrbracket_{Z, \varepsilon}$ of a $(p, q)$-tangle forms a complex of $\left(A^{(q, \varepsilon)}, A^{(p, \varepsilon)}\right)$-bimodules.

\subsection{Obtaining Khovanov's complex from Lee's and Bar-Natan's}

In this section, we show in which cases one can compute Khovanov's chain complex from Lee's or Bar-Natan's, respectively, by exploiting their filtration and passing to the associated graded complex (Section [2.7). For links, Lee and Rasmussen $[12,18]$ have shown that the filtration on the chain complex constructed with Lee's Frobenius algebra $C_{0,1}$ gives rise to a spectral sequence whose $E_{0}$-page, i.e. the associated graded complex, is Khovanov's chain complex, and whose $E_{1}$ page is Khovanov's link homology. All pages $E_{j}, j \geq 1$, are invariant under Reidemeister moves up to isomorphism. Turner [13] has shown the corresponding result for the chain complex constructed with Bar-Natan's Frobenius algebra $C_{1,0}$. The following theorem generalizes these results from links to tangles.

Theorem 3.42. Let $T$ be a plane diagram of an oriented tangle and $\varepsilon \in\{-1,+1\}$ be a checkerboard colouring. Let $\mathbb{A}=\left(A, C, \imath, \imath^{*}\right)$ be an Euler-filtered knowledgeable Frobenius algebra and $\mathbb{A}^{\prime}=\left(A^{\prime}, C^{\prime}, \imath^{\prime}, \imath^{\prime *}\right)$ be its associated Euler-graded knowledgeable Frobenius algebra (as in Remark 3.15] such that $C^{\prime}=C_{0,0}$ agrees with Khovanov's Frobenius algebra. Denote by $Z$ and $Z^{\prime}$ the open-closed TQFTs associated with $\mathbb{A}$ and $\mathbb{A}^{\prime}$, respectively.

The filtration on the tangle complex $\llbracket T \rrbracket_{\varepsilon, Z}$ gives rise to a spectral sequence whose $E_{0}$-page is the graded complex $\llbracket T \rrbracket_{\varepsilon, Z^{\prime}}$. All pages $E_{j}, j \geq 1$, of this spectral sequence are invariant under Reidemeister moves up to isomorphism.

Remark 3.43. Examples for such pairs $\left(\mathbb{A}, A^{\prime}\right)$ of knowledgeable Frobenius algebras are given by Examples 3.13 and [3.6] by Examples [3.25 and 3.8 or by any of the algebras of Theorem [3.20 (2.) and its associated Euler-graded knowledgeable Frobenius algebra.

For the proof of the theorem, we need one lemma. In order to be able to state it, we recall the following facts, see, for example [20]: Let $C$ and $C^{\prime}$ be filtered complexes and $f: C \rightarrow C^{\prime}$ be a morphism of filtered complexes, i.e. a morphism of complexes each of whose components is filtered 
of degree 0. Denote by $\left\{E_{n}\right\}_{n}$ and $\left\{E_{n}^{\prime}\right\}_{n}$ the spectral sequences induced by the filtrations of $C$ and $C^{\prime}$, respectively. Then the map $f$ induces a morphism of spectral sequences $\left\{f_{n}\right\}_{n}:\left\{E_{n}\right\}_{n} \rightarrow$ $\left\{E_{n}^{\prime}\right\}_{n}$. This means that for each page $n \geq 0$, there is a morphism of bigraded differential modules $f_{n}: E_{n} \rightarrow E_{n}^{\prime}$, i.e. a graded linear map of bidegree $(0,0)$ such that $f_{n} \circ d_{n}=d_{n}^{\prime} \circ f_{n}$, and it means that each $f_{n+1}$ is the map that $f_{n}$ induces on homology.

Lemma 3.44 (Theorem 3.4 of [20]). Let $C$ and $C^{\prime}$ be filtered complexes and $f: C \rightarrow C^{\prime}$ be a morphism of filtered complexes. Denote the induced morphism of spectral sequences by $\left\{f_{n}\right\}_{n}:\left\{E_{n}\right\}_{n} \rightarrow\left\{E_{n}^{\prime}\right\}_{n}$. If $f_{m}: E_{m} \rightarrow E_{m}^{\prime}$ is an isomorphism of bigraded differential modules for some $m$, then so is $f_{j}$ for any $j \geq m$.

Notice that it suffices to show that $f_{m}$ is a linear isomorphism. It is already linear and graded of bidegree $(0,0)$ and compatible with the differential since it is part of a morphism of spectral sequences.

Proof of Theorem 3.42. Consider the tangle complex $\llbracket T \rrbracket_{\varepsilon, Z}$ with its filtration. We first show that the associated graded complex is $\llbracket T \rrbracket_{\varepsilon, Z^{\prime}}$. The knowledgeable Frobenius algebras $\mathbb{A}$ and $\mathbb{A}^{\prime}$ have the same underlying graded vector spaces, and so both $\llbracket T \rrbracket_{\varepsilon, Z}$ and $\llbracket T \rrbracket_{\varepsilon, Z^{\prime}}$ have the same terms. We now compute the differential of the associated graded complex of the filtered complex $\llbracket T \rrbracket_{\varepsilon, Z}$ and show that it agrees with the differential of $\llbracket T \rrbracket_{\varepsilon, Z^{\prime}}$.

From the definition of the total complex $C_{\text {tot }}$ (Definition 2.23), we see that we can do this for each term of the direct sum and thereby for each smoothing $\alpha \subseteq \mathbf{n}$ separately. The differential is in each case either +1 or -1 times the linear map associated with one of the open-closed cobordisms of (3.48) to (3.53). Using the knowledgeable Frobenius algebra $\mathbb{A}=\left(A, C, \imath, \imath^{*}\right)$, these linear maps are (up to permutations of the tensor factors and up to tensoring with identities):

$$
\begin{gathered}
\left(\mu_{A} \otimes \operatorname{id}_{A}\right) \circ\left(\operatorname{id}_{A} \otimes \tau_{A, A}\right) \circ\left(\Delta_{A} \otimes \operatorname{id}_{A}\right), \\
\left(\operatorname{id}_{A} \otimes \mu_{A}\right) \circ\left(\tau_{A, A} \otimes \operatorname{id}_{A}\right) \circ\left(\operatorname{id}_{A} \otimes \Delta_{A}\right), \\
\left(\imath^{*} \otimes \operatorname{id}_{A}\right) \circ \Delta_{A}, \\
\mu_{A} \circ\left(\imath \otimes \operatorname{id}_{A}\right), \\
\mu_{C}, \\
\Delta_{C} .
\end{gathered}
$$

For each of these compositions, the associated graded map agrees with the same expression in the knowledgeable Frobenius algebra $\mathrm{A}^{\prime}$. This shows that the associated graded complex and thereby the $E_{0}$-page of the spectral sequence is $\llbracket T \rrbracket_{\varepsilon, Z^{\prime}}$.

Now we show that all pages $E_{j}, j \geq 1$, of this spectral sequence are invariant under Reidemeister moves up to isomorphism.

Let $T$ and $T^{\prime}$ be plane diagrams of oriented tangles that are related by a Reidemeister move, let $\varepsilon \in\{-1,+1\}$, and let $C:=\llbracket T \rrbracket_{\varepsilon, Z}$ and $C^{\prime}:=\llbracket T^{\prime} \rrbracket_{\varepsilon, Z}$ be the tangle complexes. These are filtered complexes. We denote by $\left\{E_{n}\right\}_{n}$ and $\left\{E_{n}^{\prime}\right\}_{n}$ the induced spectral sequences.

The invariance under Reidemeister moves of the homology of $C$ up to isomorphism is established in the proof of Theorem 3.37 by constructing chain maps $f: C \rightarrow C^{\prime}$ and $g: C^{\prime} \rightarrow C$ which form a homotopy equivalence. These chain maps are constructed from open-closed cobordisms by applying $Z$. The cobordisms are displayed, for example, in [2] and in Section 4 below.

First, both $f$ and $g$ are homomorphisms of filtered complexes. This can be seen in a case by case inspection, taking all the grading shifts in the definition (3.55) into account. Denote by $\left\{f_{n}\right\}_{n}:\left\{E_{n}\right\}_{n} \rightarrow\left\{E_{n}^{\prime}\right\}_{n}$ and by $\left\{g_{n}\right\}_{n}:\left\{E_{n}^{\prime}\right\}_{n} \rightarrow\left\{E_{n}\right\}_{n}$ the induced morphisms of spectral sequences.

Second, on the $E_{0}$-pages, the $f_{0}: E_{0} \rightarrow E_{0}^{\prime}$ and $g_{0}: E_{0}^{\prime} \rightarrow E_{0}$ are the associated graded maps of $f: C \rightarrow C^{\prime}$ and $g: C^{\prime} \rightarrow C$, respectively. By a similar argument as above, $f_{0}$ agrees with the chain map that is obtained from the same open-closed cobordism as $f$, but using the openclosed TQFT $Z^{\prime}$ rather than $Z$, and similarly for $g_{0}$. The morphisms of differential bigraded modules $f_{0}: E_{0} \rightarrow E_{0}^{\prime}$ and $g_{0}: E_{0}^{\prime} \rightarrow E_{0}$ therefore form a homotopy equivalence between the 
associated graded complexes. This is the same homotopy equivalence that appears in the proof of Reidemeister move invariance for $Z^{\prime}$ instead of $Z$. Therefore both maps induce mutually inverse maps on homology. The homology of the associated graded complexes, however, is contained in the $E_{1}$-pages (c.f. (2.51) $)$, and therefore the morphisms of bigraded differential modules $f_{1}: E_{1} \rightarrow E_{1}^{\prime}$ and $g_{1}: E_{1}^{\prime} \rightarrow E_{1}$ are mutually inverse.

By Lemma 3.44 this implies that each $f_{j}: E_{j} \rightarrow E_{j}^{\prime}, j \geq 1$, is an isomorphism.

\section{Remark 3.45.}

1. The generalization from links to tangles of Lee's [12] and Turner's [13] 'endomorphism of the Khovanov invariant' is contained in the spectral sequence, namely in the differentials $d_{j}: E_{j} \rightarrow E_{j}^{\prime}$ of the pages $j \geq 1$.

2. Already the simplest example $\mathbf{A}=\left(A, C, \imath, \imath^{*}\right)$ of Example 3.13 for a field of characteristic 2 is quite powerful. Firstly, by the above theorem, it is rich enough to recover Khovanov homology in characteristic 2. Secondly, on links, it agrees with Bar-Natan's chain complex in characteristic 2 which is, by a result of Mackaay, Tuner, and Vaz [21], sufficient to compute Rasmussen's $s$-invariant [18]. This invariant is strong enough to tell that some knots which are known to be topologically slice, are not smoothly slice [21,22]. Finally, A has a strongly separable algebra $A$, and so all of the tangle composition technology developed in the following section, will be available, too.

3. In Section 4 below, we show how one can compute the tangle complex for Lee's and BarNatan's Frobenius algebras by composing arcs and crossings and that one can then in principle compute the Khovanov version of the tangle complex from Theorem 3.42 In order to prove Theorem 3.42 however, one still needs Theorem 3.37 i.e. the global version of the proof of Reidemeister move invariance. So although some computations can be done entirely locally, the proofs that they are valid cannot.

\subsection{Examples}

In this section, we show that even the tangle complex defined by the open-closed TQFT $Z_{\mathrm{BN}}$ associated with the knowledgeable Frobenius algebra $\mathbb{A}_{\mathrm{BN}}=\left(A_{1,0}, C_{1,0}, \imath, \imath^{*}\right)$ of Example $\mathbf{3 . 1 3}$ in characteristic 2 for $t=0$ detects whether the two strands of a $(2,2)$-tangle are non-trivially braided or not. We therefore compute the tangle homology and the tangle polynomials of the complexes

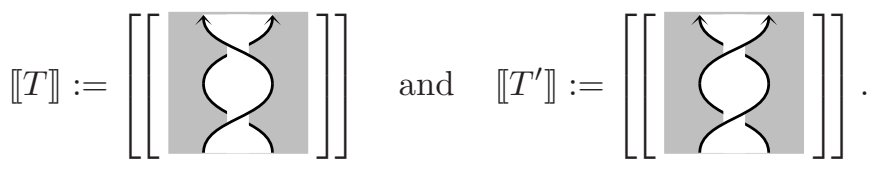

By the up to homotopy invariance of the complex $C:=\llbracket T \rrbracket$ under Reidemeister move two, the first tangle only has nontrivial homology in cohomological degree zero

$$
\mathcal{H}^{0}(C)=(A \otimes A)\{0\}
$$

and so

$$
\mathcal{P}_{Z_{\mathrm{BN}}}(T)=t^{0}\left(A^{2}+A^{-2}\right)=A^{2}+A^{-2} .
$$

The second complex $C^{\prime}:=\llbracket T^{\prime} \rrbracket$ is the filtered complex

$$
0 \longrightarrow(A \otimes A)\{4\} \stackrel{d^{0}}{\longrightarrow}(A \otimes A)\{6\} \oplus(A \otimes A)\{6\} \stackrel{d^{1}}{\longrightarrow}(A \otimes C \otimes A)\{8\} \longrightarrow 0
$$

where

$$
\begin{aligned}
& d^{0}=\left(\begin{array}{l}
\left(\operatorname{id}_{A} \otimes \mu_{A}\right) \circ\left(\tau_{A, A} \otimes \mathrm{id}_{A}\right) \circ\left(\operatorname{id}_{A} \otimes \Delta_{A}\right) \\
\left(\operatorname{id}_{A} \otimes \mu_{A}\right) \circ\left(\tau_{A, A} \otimes \mathrm{id}_{A}\right) \circ\left(\operatorname{id}_{A} \otimes \Delta_{A}\right)
\end{array}\right) \\
& d^{1}=\left(\left(\left(\operatorname{id}_{A} \otimes \imath^{*}\right) \circ \Delta_{A}\right) \otimes \operatorname{id}_{A}, \operatorname{id}_{A} \otimes\left(\left(\imath^{*} \otimes \mathrm{id}_{A}\right) \circ \Delta_{A}\right)\right)
\end{aligned}
$$


The kernel of $d^{0}$ is the filtered vector space $\langle 1 \otimes y+y \otimes 1,1 \otimes y+y \otimes y\rangle\{4\}$. A simple calculation reveals that

$$
\operatorname{im} d^{0}=\operatorname{ker} d^{1}=\langle(1 \otimes 1+1 \otimes y+y \otimes 1) \oplus(1 \otimes 1+1 \otimes y+y \otimes 1),(y \otimes y) \oplus(y \otimes y)\rangle\{4\} .
$$

Furthermore,

$$
\begin{aligned}
\operatorname{im} d^{1=} & \langle 1 \otimes x \otimes y, y \otimes x \otimes 1, y \otimes x \otimes y, 1 \otimes 1 \otimes 1+1 \otimes x \otimes 1+y \otimes 1 \otimes 1, \\
& 1 \otimes 1 \otimes 1+1 \otimes x \otimes 1+1 \otimes 1 \otimes y, 1 \otimes 1 \otimes y+y \otimes 1 \otimes y\rangle\{8\} .
\end{aligned}
$$

Therefore,

$$
\mathcal{H}^{n}\left(C^{\prime}\right)= \begin{cases}\langle 1 \otimes y+y \otimes 1,1 \otimes y+y \otimes y\rangle\{4\} & \text { if } n=0, \\ \langle 1 \otimes 1 \otimes 1,1 \otimes x \otimes 1\rangle\{8\} & \text { if } n=2, \\ 0 & \text { otherwise. }\end{cases}
$$

Taking the grading shifts into account, we obtain the following bigrading for this homology:

$$
\begin{aligned}
\mathcal{H}^{2,0}\left(C^{\prime}\right) & =\langle 1 \otimes y+y \otimes y\rangle, \\
\mathcal{H}^{4,0}\left(C^{\prime}\right) & =\langle 1 \otimes y+y \otimes 1\rangle, \\
\mathcal{H}^{8,2}\left(C^{\prime}\right) & =\langle 1 \otimes x \otimes 1\rangle, \\
\mathcal{H}^{12,2}\left(C^{\prime}\right) & =\langle 1 \otimes 1 \otimes 1\rangle .
\end{aligned}
$$

Hence, the tangle polynomial for $T^{\prime}$ is

$$
\mathcal{P}_{Z_{\mathrm{BN}}}\left(T^{\prime}\right)=t^{0}\left(A^{2}+A^{4}\right)+t^{2}\left(A^{8}+A^{12}\right)=A^{2}+A^{4}+t^{2} A^{8}+t^{2} A^{12} .
$$

Computing the homology of the associated graded complex for $\llbracket T^{\prime} \rrbracket$, i.e. using the open-closed TQFT $Z_{\mathrm{Kh}}$ associated with the knowledgeable Frobenius algebra of Example 3.6] we get

$$
\begin{aligned}
\mathcal{H}^{2,0}\left(C^{\prime}\right) & =\langle y \otimes y\rangle, \\
\mathcal{H}^{4,0}\left(C^{\prime}\right) & =\langle 1 \otimes y+y \otimes 1\rangle, \\
\mathcal{H}^{6,1}\left(C^{\prime}\right) & =\left\langle\left(\begin{array}{c}
1 \otimes y \\
1 \otimes y
\end{array}\right)\right\rangle, \\
\mathcal{H}^{8,1}\left(C^{\prime}\right) & =\left\langle\left(\begin{array}{c}
1 \otimes 1 \\
1 \otimes 1
\end{array}\right)\right\rangle, \\
\mathcal{H}^{8,2}\left(C^{\prime}\right) & =\langle y \otimes 1 \otimes y\rangle, \\
\mathcal{H}^{10,2}\left(C^{\prime}\right) & =\langle 1 \otimes 1 \otimes y, y \otimes 1 \otimes 1\rangle, \\
\mathcal{H}^{12,2}\left(C^{\prime}\right) & =\langle 1 \otimes 1 \otimes 1\rangle,
\end{aligned}
$$

so that

$$
\mathcal{P}_{Z_{\mathrm{Kh}}}\left(T^{\prime}\right)=A^{4}+A^{2}+t^{1}\left(A^{8}+q^{6}\right)+t^{2}\left(A^{12}+2 A^{10}+A^{8}\right) .
$$

\section{Composition of tangles}

\subsection{Tensor products of complexes of bimodules}

Let us first introduce an efficient way of describing tensor products of complexes whose terms are bimodules.

Let $A$ and $B$ be $k$-algebras and $C=\left(C^{i}, d^{i}\right)$ be a complex of $(A, B)$-bimodules, i.e. an object of $\operatorname{Kom}\left({ }_{A} \mathcal{M}_{B}\right)$. Denote the $(A, B)$-bimodule structure of each $C^{i}$ by $\lambda^{i}: A \otimes C^{i} \rightarrow C^{i}$ and $\rho^{i}: C^{i} \otimes B \rightarrow C^{i}$. If we view $A$ as a 1 -term complex $0 \rightarrow A \rightarrow 0$, then the tensor product of complexes $A \otimes C$ has the terms $(A \otimes C)^{i}=A \otimes C^{i}$ and the differential $d_{A \otimes C}^{i}=\mathrm{id}_{A} \otimes d^{i}$. The 
$\left\{\lambda^{i}\right\}_{i}$ form a morphism of complexes $\lambda: A \otimes C \rightarrow C$, i.e. a morphism of $\mathbf{K o m}\left(\operatorname{Vect}_{k}\right)$. Similarly, there is a morphism of complexes $\rho: C \otimes B \rightarrow C$.

If both $C$ and $D$ are complexes of $(A, B)$-bimodules with $\lambda_{C}: A \otimes C \rightarrow C, \rho_{C}: C \otimes B \rightarrow C$, $\lambda_{D}: A \otimes D \rightarrow D$, and $\rho_{D}: D \otimes B \rightarrow D$ defined as above, and if $f: C \rightarrow D$ is a morphism of such complexes, then we have $f \circ \lambda_{C}=\lambda_{D} \circ\left(\operatorname{id}_{A} \otimes f\right)$ and $f \circ \rho_{C}=\rho_{D} \circ\left(f \otimes \operatorname{id}_{B}\right)$.

Lemma 4.1. Let $A, B$, and $B^{\prime}$ be $k$-algebras and $C=\left(C^{i}, d^{i}\right)$ be a complex of $\left(A \otimes B^{\prime}, A \otimes B^{\prime \prime}\right)$ bimodules, i.e. an object of $\operatorname{Kom}\left(A \otimes B^{\prime} \mathcal{M}_{A \otimes B^{\prime \prime}}\right)$. Then the following coequalizer in $\operatorname{Kom}\left(B^{\prime} \mathcal{M}_{B^{\prime \prime}}\right)$,

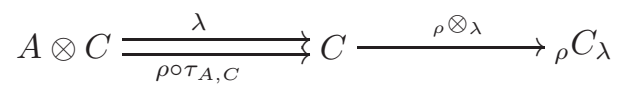

exists. We have denoted by $\lambda: A \otimes C \rightarrow C$ and $\rho: C \otimes A \rightarrow C$ the maps that make $C$ a complex of $(A, A)$-bimodules. These are morphisms of $\operatorname{Kom}\left(B^{\prime} \mathcal{M}_{B^{\prime \prime}}\right)$.

The complex ${ }_{\rho} C_{\lambda}=\left({ }_{\rho} C_{\lambda}^{i}, d_{\rho}^{i} C_{\lambda}\right)$ is the complex whose terms are the coequalizers

$$
A \otimes C^{i} \underset{\rho^{i} \circ \tau_{A, C}}{\stackrel{\lambda^{i}}{\longrightarrow}} C^{i} \stackrel{\otimes_{\lambda}^{i}}{\longrightarrow} C_{\lambda}^{i}
$$

in the category ${ }_{B^{\prime}} \mathcal{M}_{B^{\prime \prime}}$ and whose differential $d_{\rho}^{i} C_{\lambda}$ is uniquely specified as the morphism in the commutative diagram

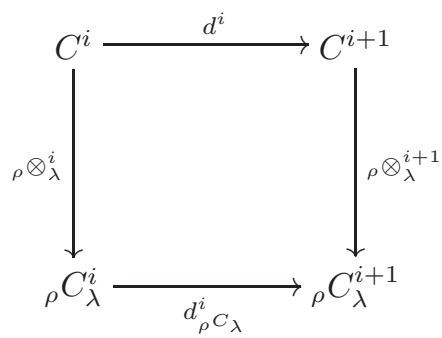

because by the universal property, $\rho \otimes_{\lambda}^{i+1} \circ d^{i}$ factors uniquely through the coequalizer $\rho_{\lambda} \otimes_{\lambda}^{i}$. The coequalizer map $\rho_{\lambda}$ in (4.1) is the morphism of $\operatorname{Kom}\left({ }_{B}^{\prime} \mathcal{M}_{B^{\prime \prime}}\right)$ whose components are the coequalizers $\rho \otimes_{\lambda}^{i}$ of 4.2].

\subsection{Construction of the tangle complex from constituents}

In this section, we show how one can obtain the tangle complex of Definition 3.35 from the iterated tensor product over $A$ of two basic building blocks: A 1-term complex $0 \rightarrow A \rightarrow 0$ associated with arcs and a 2-term complex $0 \rightarrow A \otimes A \rightarrow A \otimes A \rightarrow 0$ associated with crossings which comes in four different variations, depending on the orientations and on the checkerboard colouring. The requirement is that we employ a state sum knowledgeable Frobenius algebra $\left(A, Z(A), \imath, \imath^{*}\right)$ that satisfies Bar-Natan's conditions. Warning: In this section, $C$ denotes a complex whereas $Z(A)$ is the relevant commutative Frobenius algebra.

Definition 4.2. Let $T$ be a plane diagram of an oriented $(p, q)$-tangle with $n_{+}$positive ( $~(x)$ and $n_{-}$negative crossings $(\lambda), n:=n_{+}+n_{-}$; let $\varepsilon \in\{-1,+1\}$ denote a checkerboard colouring and let $\mathbb{A}=\left(A, Z(A), \imath, \imath^{*}\right)$ be an [Euler-graded, Euler-filtered] state sum knowledgeable Frobenius algebra that satisfies Bar-Natan's conditions.

Choose a finite graph $G \subseteq \mathbb{R}^{2}$ embedded in the plane such that

- the vertices of $G$ do not intersect $T$, and

- the intersection $G \cap T$ is a finite set, and

- the closure $\overline{R_{j}}$ of each connected component $R_{1}, \ldots, R_{\ell}$ of the complement $\mathbb{R}^{2} \backslash G$ intersects $T$ in such a way that $\overline{R_{j}} \cap T$ is either an arc (|) or a crossing (X). 
Number the boundary points of the $\overline{R_{j}} \cap T$ by $1, \ldots, k$, i.e. $k=4 n+2 m$ where $m$ is the number of arcs among the $\overline{R_{j}} \cap T$. For each $j \in\{1, \ldots, \ell\}$, we define a complex $C_{j}$ as follows.

1. If $\overline{R_{j}} \cap T$ is an $\operatorname{arc}(\mid)$, we set

$$
C_{j}:=0 \longrightarrow \underline{A} \longrightarrow 0
$$

where we have underlined the term in cohomological degree 0 . Let $i_{1}, i_{2} \in\{1, \ldots, k\}$ be the numbers of the boundary points of the arc. One of them, say $i_{1}$, is a '-'-boundary relative to $\varepsilon$ (c.f. Proposition 3.40). The complex $C_{j}$ above is a complex of left $A$-modules whose left $A$-action we denote by $\lambda^{\left(i_{1}\right)}: A \otimes C_{j} \rightarrow C_{j}$. The other point numbered $i_{2}$ is a '+'-boundary, and we denote the right $A$-action by $\rho^{\left(i_{2}\right)}: C_{j} \otimes A \rightarrow C_{j}$.

2. If $\overline{R_{j}} \cap T$ is a positive crossing with the checkerboard colouring

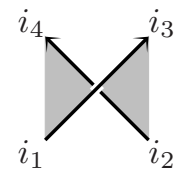

whose boundary points are numbered $i_{1}, i_{2}, i_{3}, i_{4} \in\{1, \ldots, k\}$ as indicated, we define

$$
C_{j}:=0 \longrightarrow \underline{A \otimes A\{2\}} \stackrel{\left(\mu_{A} \otimes \mathrm{id}_{A}\right) \circ\left(\mathrm{id}_{A} \otimes \tau_{A, A}\right) \circ\left(\Delta_{A} \otimes \mathrm{id}_{A}\right)}{\longrightarrow} A \otimes A\{4\} \longrightarrow 0 .
$$

This is a [graded, filtered] 2-term complex of $(A \otimes A, A \otimes A)$-bimodules. We write

$$
\begin{array}{llll}
\lambda_{1}: A \otimes(A \otimes A) \rightarrow A \otimes A, & a \otimes(x \otimes y) & \mapsto & \mu_{A}(a \otimes x) \otimes y, \\
\lambda_{2}: A \otimes(A \otimes A) \rightarrow A \otimes A, & a \otimes(x \otimes y) & \mapsto x \otimes \mu_{A}(a \otimes y), \\
\rho_{1}:(A \otimes A) \otimes A \rightarrow A \otimes A, & (x \otimes y) \otimes a & \mapsto \mu_{A}(x \otimes a) \otimes y, \\
\rho_{2}:(A \otimes A) \otimes A \rightarrow A \otimes A, & (x \otimes y) \otimes a & \mapsto x \otimes \mu_{A}(y \otimes a)
\end{array}
$$

for the two left and the two right actions of $A$ on $A \otimes A$. The structure of $C_{j}$ as a complex of $(A \otimes A, A \otimes A)$-bimodules is then given by the following four actions. As above, '-'boundaries relative to $\varepsilon$ result in left actions and ' + '-boundaries in right actions:

(a) $\lambda^{\left(i_{1}\right)}: A \otimes C_{j} \rightarrow C_{j}$ which is defined by $\left(\lambda^{\left(i_{1}\right)}\right)^{0}:=\lambda_{1}$ and $\left(\lambda^{\left(i_{1}\right)}\right)^{1}:=\lambda_{1}$ in degree 0 and 1 , respectively.

(b) $\rho^{\left(i_{2}\right)}: C_{j} \otimes A \rightarrow C_{j}$ with $\left(\rho^{\left(i_{2}\right)}\right)^{0}:=\rho_{2}$ and $\left(\rho^{\left(i_{2}\right)}\right)^{1}:=\rho_{1}$.

(c) $\lambda^{\left(i_{3}\right)}: A \otimes C_{j} \rightarrow C_{j}$ with $\left(\lambda^{\left(i_{3}\right)}\right)^{0}:=\lambda_{2}$ and $\left(\lambda^{\left(i_{3}\right)}\right)^{1}:=\lambda_{2}$.

(d) $\rho^{\left(i_{4}\right)}: C_{j} \otimes A \rightarrow C_{j}$ with $\left(\rho^{\left(i_{4}\right)}\right)^{0}:=\rho_{1}$ and $\left(\rho^{\left(i_{4}\right)}\right)^{1}:=\rho_{2}$.

3. If $\overline{R_{j}} \cap T$ is a negative crossing with the checkerboard colouring

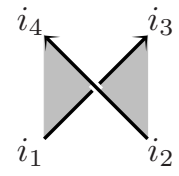

we define

$$
C_{j}:=0 \longrightarrow A \otimes A\{-4\} \stackrel{\left(\mu_{A} \otimes \mathrm{id}_{A}\right) \circ\left(\mathrm{id}_{A} \otimes \tau_{A, A}\right) \circ\left(\Delta_{A} \otimes \mathrm{id}_{A}\right)}{\longrightarrow} \underline{A \otimes A\{-2\}} \longrightarrow 0 .
$$

The $A$-actions are:

(a) $\lambda^{\left(i_{1}\right)}: A \otimes C_{j} \rightarrow C_{j}$ with $\left(\lambda^{\left(i_{1}\right)}\right)^{-1}:=\lambda_{1}$ and $\left(\lambda^{\left(i_{1}\right)}\right)^{0}:=\lambda_{1}$,

(b) $\rho^{\left(i_{2}\right)}: C_{j} \otimes A \rightarrow C_{j}$ with $\left(\rho^{\left(i_{2}\right)}\right)^{-1}:=\rho_{1}$ and $\left(\rho^{\left(i_{2}\right)}\right)^{0}:=\rho_{2}$, 
(c) $\lambda^{\left(i_{3}\right)}: A \otimes C_{j} \rightarrow C_{j}$ with $\left(\lambda^{\left(i_{3}\right)}\right)^{-1}:=\lambda_{2}$ and $\left(\lambda^{\left(i_{3}\right)}\right)^{0}:=\lambda_{2}$,

(d) $\rho^{\left(i_{4}\right)}: C_{j} \otimes A \rightarrow C_{j}$ with $\left(\rho^{\left(i_{4}\right)}\right)^{-1}:=\rho_{2}$ and $\left(\rho^{\left(i_{4}\right)}\right)^{0}:=\rho_{1}$.

4. If $\overline{R_{j}} \cap T$ is a positive crossing with the checkerboard colouring

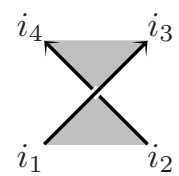

we define

$$
C_{j}:=0 \longrightarrow \underline{A \otimes A\{2\}} \stackrel{\left(\mathrm{id}_{A} \otimes \mu_{A}\right) \circ\left(\tau_{A}, A \otimes \mathrm{id}_{A}\right) \circ\left(\mathrm{id}_{A} \otimes \Delta_{A}\right)}{\longrightarrow} A \otimes A\{4\} \longrightarrow 0 .
$$

The $A$-actions are:

(a) $\rho^{\left(i_{1}\right)}: C_{j} \otimes A \rightarrow C_{j}$ with $\left(\rho^{\left(i_{1}\right)}\right)^{0}:=\rho_{1}$ and $\left(\rho^{\left(i_{1}\right)}\right)^{1}:=\rho_{1}$,

(b) $\lambda^{\left(i_{2}\right)}: A \otimes C_{j} \rightarrow C_{j}$ with $\left(\lambda^{\left(i_{2}\right)}\right)^{0}:=\lambda_{2}$ and $\left(\lambda^{\left(i_{2}\right)}\right)^{1}:=\lambda_{1}$,

(c) $\rho^{\left(i_{3}\right)}: C_{j} \otimes A \rightarrow C_{j}$ with $\left(\rho^{\left(i_{3}\right)}\right)^{0}:=\rho_{2}$ and $\left(\rho^{\left(i_{3}\right)}\right)^{1}:=\rho_{2}$,

(d) $\lambda^{\left(i_{4}\right)}: A \otimes C_{j} \rightarrow C_{j}$ with $\left(\lambda^{\left(i_{4}\right)}\right)^{0}:=\lambda_{1}$ and $\left(\lambda^{\left(i_{4}\right)}\right)^{1}:=\lambda_{2}$.

5. If $\overline{R_{j}} \cap T$ is a negative crossing with the checkerboard colouring

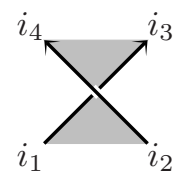

we define

$$
C_{j}:=0 \longrightarrow A \otimes A\{-4\} \stackrel{\left(\mathrm{id}_{A} \otimes \mu_{A}\right) \circ\left(\tau_{A, A} \otimes \mathrm{id}_{A}\right) \circ\left(\mathrm{id}_{A} \otimes \Delta_{A}\right)}{\longrightarrow} \underline{A \otimes A\{-2\}} \longrightarrow 0 .
$$

The $A$-actions are:
(a) $\rho^{\left(i_{1}\right)}: C_{j} \otimes A \rightarrow C_{j}$ with $\left(\rho^{\left(i_{1}\right)}\right)^{-1}:=\rho_{1}$ and $\left(\rho^{\left(i_{1}\right)}\right)^{0}:=\rho_{1}$,
(b) $\lambda^{\left(i_{2}\right)}: A \otimes C_{j} \rightarrow C_{j}$ with $\left(\lambda^{\left(i_{2}\right)}\right)^{-1}:=\lambda_{1}$ and $\left(\lambda^{\left(i_{2}\right)}\right)^{0}:=\lambda_{2}$,
(c) $\rho^{\left(i_{3}\right)}: C_{j} \otimes A \rightarrow C_{j}$ with $\left(\rho^{\left(i_{3}\right)}\right)^{-1}:=\rho_{2}$ and $\left(\rho^{\left(i_{3}\right)}\right)^{0}:=\rho_{2}$,
(d) $\lambda^{\left(i_{4}\right)}: A \otimes C_{j} \rightarrow C_{j}$ with $\left(\lambda^{\left(i_{4}\right)}\right)^{-1}:=\lambda_{2}$ and $\left(\lambda^{\left(i_{4}\right)}\right)^{0}:=\lambda_{1}$.

Let $G \cap T=\left\{x_{1}, \ldots, x_{s}\right\}$ be the set of points at which the graph $G$ intersects the tangle diagram $T$ and thereby 'cuts' it into its constituents. For each $i \in\{1, \ldots, s\}$, there are two numbers $p_{i}, q_{i} \in\{1, \ldots, k\}$ indicating the numbers of the two boundary points of some of the $\overline{R_{j}} \cap T$ that coincide with $x_{i}$. One of them, say $p_{i}$ is a '+'-boundary and thus corresponds to a right $A$-action $\rho^{\left(p_{i}\right)}$ on some $C_{j}$, whereas the other one, $q_{i}$, is a '-'-boundary relative to $\varepsilon$ and therefore corresponds to a left $A$-action $\lambda^{\left(q_{i}\right)}$ on some $C_{j^{\prime}}$.

We now take the tensor product over $k$ of all complexes $C_{j}, j \in\{1, \ldots, \ell\}$, and then apply the coequalizer that 'coequalizes' the right $A$-action $\rho^{\left(p_{i}\right)}$ with the left $A$-action $\lambda^{\left(q_{i}\right)}$ for all $i \in$ $\{1, \ldots, s\}$ :

$$
C(T, \varepsilon, \mathbb{A}):=\rho_{\rho^{\left(p_{1}\right)}} \otimes_{\lambda^{\left(q_{1}\right)}}\left({ }_{\rho^{\left(p_{2}\right)}} \otimes_{\lambda^{\left(q_{2}\right)}}\left(\cdots\left({ }_{\rho^{\left(p_{s}\right)}} \otimes_{\lambda^{\left(q_{s}\right)}}\left(\bigotimes_{j=1}^{\ell} C_{j}\right)\right) \cdots\right)\right) .
$$


Remark 4.3. In each of the five cases, $C_{j}$ agrees with the tangle complex $\left[\left[\overline{R_{j}} \cap T\right]\right]_{\varepsilon, Z}$ for the state sum open-closed TQFT $Z$ associated with A. Note in particular that the differential of (4.6) agrees with $Z(-)$ applied to the open-closed cobordism (3.48), etc.. For each of the complexes, we have in detail specified the $A$-actions associated with the boundary points (c.f. Proposition 3.40). The complex $C(T, \varepsilon, \mathbb{A})$ is a complex of $\left(A^{\otimes r}, A^{\otimes r}\right)$-bimodules, $p+q=2 r$, associated with the diagram of the $(p, q)$-tangle $T$.

Theorem 4.4. Let $T$ be a plane diagram of an oriented $(p, q)$-tangle, $\varepsilon \in\{-1,+1\}$ denote a checkerboard colouring, and let $Z: \mathbf{2} \mathbf{C o b}^{\text {ext }} \rightarrow$ Vect $_{k}$ be a [Euler-graded, Euler-filtered] state sum open-closed TQFT that satisfies Bar-Natan's conditions. We denote by A its associated knowledgeable Frobenius algebra. Then the complex of Definition 4.2 is isomorphic to the tangle complex of Definition 3.5.5]

$$
C(T, \varepsilon, \mathbb{A}) \cong \llbracket T \rrbracket_{\varepsilon, Z},
$$

as [graded, filtered] complexes of $\left(A^{(q, \varepsilon)}, A^{(p, \varepsilon)}\right)$-bimodules.

While the proof of this theorem is conceptually simple, making a precise proof is rather laborious. The picture that the reader should have in mind is that the complex constructed via coequalizers is actually what one would get by using the state sum construction of [17]. Although the proof below is entirely self contained, it really just amounts to showing that the state sum construction actually reproduces the knowledgeable Frobenius algebra associated to the Euler-graded [Euler-filtered] state sum open-closed TQFT $Z$. As in [17], the key to proving this theorem will be the maps $P_{i j}$ and $Q_{i j}$ corresponding to triangulated strips and cylinders whose incoming boundary are triangulated with $i$ arcs and whose outgoing boundaries are triangulated with $j$ arcs.

Proof. First, we observe that each of the complexes $C_{j}, j \in\{1, \ldots, \ell\}$, of (4.4), 4.6), (4.12), (4.14), and (4.16) is the total complex $C_{j} \cong C_{\text {tot }}\left(\mathcal{I}_{j}, \widetilde{C}_{j}\right)$ of some commutative cube $\widetilde{C}_{j}$ in Vect $\left[\operatorname{grdVect}_{k}\right.$, fltVect $\left._{k}\right]$. For (4.4), this is an $\mathcal{I}_{j}$-cube with only one vertex, i.e. $\mathcal{I}_{j}=\emptyset$, whereas in the other cases, this is an $\mathcal{I}_{j}$-cube with two vertices, i.e. $\mathcal{I}=\{z\}$ where $z \in \alpha \subseteq \mathbf{n}$ indicates the number of the respective crossing. By Proposition 2.24

$$
\bigotimes_{j=1}^{\ell} C_{j} \cong C_{\mathrm{tot}}(\mathbf{n}, \widetilde{C})\left\{2 n_{+}-4 n_{-}\right\} \quad \text { where } \quad \widetilde{C}:=\bigotimes_{j=1}^{\ell} \widetilde{C}_{j},
$$

i.e. the tensor product of complexes that features in 4.17), is the total complex of the external tensor product of the commutative cubes $\widetilde{C}_{j}$. We have made some of the grading shifts explicit here.

The vertices of the commutative cube $\widetilde{C}$ are labeled by the smoothings $\alpha \subseteq \mathbf{n}$ of the $n$ crossings of $T$. Each vertex $\widetilde{C}_{\alpha}$ of $\widetilde{C}$ is of the form $\widetilde{C}_{\alpha} \cong A^{\otimes(2 n+m)}\{2|\alpha|\}$ since each of the $n$ crossings contributes a tensor factor of $A \otimes A$ and each of the $m$ arcs contributes just an $A$. The ordering of the tensor factors is induced from the numbering $1, \ldots, \ell$ of the components of $\left(\mathbb{R}^{2} \backslash G\right) \cap T$.

In order to prove the theorem, we permute the tensor factors of each $\widetilde{C}_{\alpha} \cong A^{\otimes(2 n+m)}, \alpha \subseteq \mathbf{n}$, so that an isomorphism of commutative cubes can be explicitly given. By an argument analogous to that of Proposition 3.33 (2.), permuting the tensor factors of an arbitrary vertex of a commutative cube $\widetilde{C}$ results in applying an isomorphism to the associated total complex $C_{\text {tot }}(\mathbf{n}, \widetilde{C})$.

Consider a vertex $\alpha \subseteq \mathbf{n}$ of the cube $\widetilde{C}$. The associated smoothing $D_{\alpha} \subseteq \mathbb{R}^{2}$ of the tangle diagram is a disjoint union of arcs and circles that can be ordered arbitrarily. Number these components of $D_{\alpha}$ by $1, \ldots, k_{\alpha}$ in the same way as in Definition [3.26] and also define the sequence $m^{(\alpha)}=\left(m_{1}^{(\alpha)}, \ldots, m_{k_{\alpha}}^{(\alpha)}\right) \in\{0,1\}^{k_{\alpha}}$ such that $m_{i}^{(\alpha)}=0$ if the $i$-th component of $D_{\alpha}$ is a circle and $m_{i}^{(\alpha)}=1$ if it is an arc. The graph $G$ chops each $D_{\alpha} \subseteq \mathbb{R}^{2}$ into several pieces $\overline{R_{j}} \cap D_{\alpha}$, $j \in\{1, \ldots, \ell\}$. If $\overline{R_{j}} \cap T$ is an arc, then $\overline{R_{j}} \cap D_{\alpha}$ is an arc and $\widetilde{C}_{j} \cong A$, whereas if $\overline{R_{j}} \cap T$ is a crossing, then $\overline{R_{j}} \cap D_{\alpha}$ is the disjoint union of two arcs and $\widetilde{C}_{j} \cong A \otimes A$.

We assume that we have permuted the tensor factors of each $\widetilde{C}_{\alpha}$ in such a way that

$$
\widetilde{C}_{\alpha}=\widetilde{C}_{\alpha}^{(1)} \otimes \cdots \otimes \widetilde{C}_{\alpha}^{\left(k_{\alpha}\right)}\{2|\alpha|\},
$$


where the $\widetilde{C}_{\alpha}^{(i)}$ are defined as follows. If $m_{i}^{(\alpha)}=0$, then the $i$-th component $D_{\alpha}^{(i)}$ of $D_{\alpha}$ is a circle which is chopped by the graph complement into $M_{i}^{(\alpha)}:=\left|\left\{j \in\{1, \ldots, \ell\} \mid \overline{R_{j}} \cap D_{\alpha}^{(j)} \neq \emptyset\right\}\right| \operatorname{arcs}$. If $m_{i}^{(\alpha)}=1$, then the $i$-th component is an arc which is chopped into $M_{i}^{(\alpha)}$ arcs. In both cases, we put $\widetilde{C}_{\alpha}^{(i)}:=A^{\otimes M_{i}^{(\alpha)}}$.

We also assume that we have permuted the tensor factors of the $\widetilde{C}_{\alpha}^{(i)}$ in such a way that any pair of left and right action $\lambda^{\left(q_{t}\right)}$ and $\rho^{\left(p_{t}\right)}$ involved in the coequalizer (4.17) either

- consists of a right action on the $b$-th and a left action on the $(b+1)$-th tensor factor of some $\widetilde{C}_{\alpha}^{(i)}$ if $m_{i}^{(\alpha)} \in\{0,1\}$, or

- consists of a right action on the last and a left action on the first tensor factor of some $\widetilde{C}_{\alpha}^{(i)}$ if $m_{i}^{(\alpha)}=0$ (circle only).

The complex $C(T, \varepsilon, \mathbb{A})$ of 4.17) can now be obtained by applying the composition of coequalizers

$$
\bigotimes_{\rho ; \lambda}:={ }_{\rho^{\left(p_{1}\right)}} \otimes_{\lambda^{\left(q_{1}\right)}} \circ \cdots \circ{ }_{\rho^{\left(p_{s}\right)}} \otimes_{\lambda^{\left(q_{s}\right)}}
$$

of vector spaces to each vertex $\widetilde{C}_{\alpha}, \alpha \subseteq \mathbf{n}$ of the cube and by then taking the total complex:

$$
C(T, \varepsilon, \mathbb{A}) \cong C_{\text {tot }}\left(\mathbf{n}, \bigotimes_{\rho ; \lambda}(\widetilde{C})\right)\left\{2 n_{+}-4 n_{-}\right\} .
$$

Here we have denoted by $\bigotimes_{\rho ; \lambda}(\widetilde{C})$ the cube whose vertices are the $\bigotimes_{\rho ; \lambda}\left(\widetilde{C}_{\alpha}\right)$ and whose edges are obtained by repeatedly applying the coequalizer to maps as in (2.34).

Because of the way we have ordered the tensor factors of the $\widetilde{C}_{\alpha}^{(i)}$,

1. if $m_{i}^{(\alpha)}=1$, then the ordering of factors is precisely as in (2.27) and

$$
\bigotimes_{\rho ; \lambda}\left(\widetilde{C}_{\alpha}^{(i)}\right) \cong P_{M_{i}^{(\alpha)} M_{i}^{(\alpha)}}\left(A^{\otimes M_{i}^{(\alpha)}}\right) \cong A,
$$

2. if $m_{i}^{(\alpha)}=0$, then the ordering of factors is precisely as in (2.28) and

$$
\bigotimes_{\rho ; \lambda}\left(\widetilde{C}_{\alpha}^{(i)}\right) \cong Q_{M_{i}^{(\alpha)} M_{i}^{(\alpha)}}\left(A^{\otimes M_{i}^{(\alpha)}}\right) \cong p(A) \cong Z(A) .
$$

This shows that the vertices (4.20) of the cube $\widetilde{C}$ are isomorphic to the vertices of the cube $Z\left(S_{T, \varepsilon}\right)$ (Definition 3.29). We finally have to show that the corresponding isomorphisms fit into an isomorphism of commutative cubes which then implies that the resulting total complexes in 4.18 are isomorphic.

The edges $(\alpha, x) \in r(\mathbf{n})$ of the cube $\bigotimes_{\rho ; \lambda}(\widetilde{C})$ are obtained from those of $\widetilde{C}$ by repeatedly applying coequalizers to maps as in (2.34). As we know that the coequalizers $\bigotimes_{\rho ; \lambda}: \widetilde{C}_{\alpha}^{(i)} \rightarrow A$ or $\widetilde{C}_{\alpha}^{(i)} \rightarrow Z(A)$ are just $P_{1 M_{i}^{(\alpha)}}$ or $Q_{1 M_{i}^{(\alpha)}}$ (Corollary 2.11) that have inverses $P_{M_{i}^{(\alpha)} 1}$ or $Q_{M_{i}^{(\alpha)} 1}$ if restricted appropriately (Proposition [2.9), we can write down the edges of the cube explicitly.

If $\widetilde{C}_{(\alpha, x)}: \widetilde{C}_{\alpha} \rightarrow \widetilde{C}_{\alpha \sqcup\{x\}}, x \in \mathbf{n}, \alpha \subseteq \mathbf{n} \backslash\{x\}$, is an edge of the cube $\widetilde{C}$, i.e. $\widetilde{C}_{(\alpha, x)}$ is a tensor product of either (3.48) or (3.49) with identities, then the induced map

$$
\widehat{C}_{(\alpha, x)}:\left(\bigotimes_{\rho ; \lambda}(\widetilde{C})\right)_{\alpha} \rightarrow\left(\bigotimes_{\rho ; \lambda}(\widetilde{C})\right)_{\alpha \sqcup\{x\}},
$$

is given by

$$
\widehat{C}_{(\alpha, x)}=\left(P_{M_{1}^{(\alpha)} 1}^{\left(m_{1}^{(\alpha)}\right)} \otimes \cdots \otimes P_{M_{k_{\alpha}}^{(\alpha)} 1}^{\left(m_{\alpha}^{(\alpha)}\right)}\right) \circ \widetilde{C}_{(\alpha, x)} \circ\left(P_{1 M_{1}^{(\alpha)}}^{\left(m_{1}^{(\alpha)}\right)} \otimes \cdots \otimes P_{1 M_{k_{\alpha}}^{(\alpha)}}^{\left(m_{k_{\alpha}}^{(\alpha)}\right)}\right)
$$


where we have used the notation $P_{k \ell}^{(0)}:=Q_{k \ell}$ and $P_{k \ell}^{(1)}:=P_{k \ell}$. Recall that the composition in (1.24) is an example of such a map. We now have to verify that these edges agree with the edges of the cube $Z\left(S_{T, \varepsilon}\right)$ of Definition 3.29 i.e.

$$
\widehat{C}_{(\alpha, x)}=Z\left(S_{(\alpha, x)}\right)
$$

for all $x \in \mathbf{n}, \alpha \subseteq \mathbf{n} \backslash\{x\}$. Since we know that $\widetilde{C}_{(\alpha, x)}$ is the tensor product of either (3.48) or 3.49) with identities and since we have the explicit forms of $P_{k \ell}$ and $Q_{k \ell}$, this can be done in case by verification.

Up to permutations, duality, $A \leftrightarrow A^{\text {op }}$ symmetry and the relations (2.17), we have to verify four equations. We write $d:=\left(\mu_{A} \otimes \mathrm{id}_{A}\right) \circ\left(\operatorname{id}_{A} \otimes \tau_{A, A}\right) \circ\left(\Delta_{A} \otimes \mathrm{id}_{A}\right)$ for the differential associated with the crossing (3.48).

1. $\left(P_{12} \otimes \mathrm{id}_{A}\right) \circ\left(\operatorname{id}_{A} \otimes d\right) \circ\left(P_{21} \otimes \mathrm{id}_{A}\right)=d$. This corresponds to gluing an arc to a crossing in the trivial way and can be seen to follow from the relations of the knowledgeable Frobenius algebra as follows:

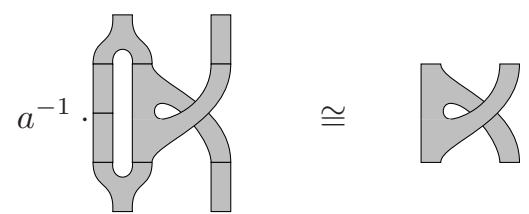

2. $\left(\operatorname{id}_{A} \otimes P_{12}\right) \circ\left(d \otimes \operatorname{id}_{A}\right) \circ\left(\operatorname{id}_{A} \otimes \tau_{A, A}\right) \circ\left(P_{21} \otimes \operatorname{id}_{A}\right)=d$. This also corresponds to gluing an arc to a crossing in the trivial way:

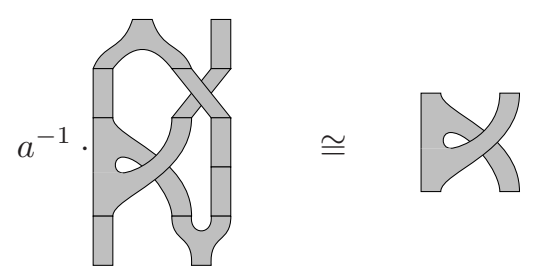

3. $P_{12} \circ \tau_{A, A} \circ d \circ\left(Q_{11} \otimes \operatorname{id}_{A}\right)=\mu_{A} \circ\left(\imath \otimes \operatorname{id}_{A}\right)$. Note that $Q_{11}=p=a^{-1} \cdot \imath$. This corresponds to an arc which is glued with both ends to two boundary points of a crossing:

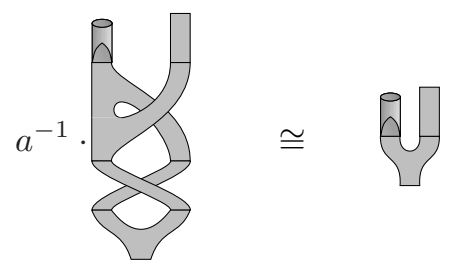

4. $Q_{11} \circ \mu_{A} \circ\left(\imath \otimes Q_{11}\right)=\mu_{Z(A)}$. Note that $Q_{11}=a^{-1} \cdot \imath$ if restricted to $p(A)$ and $Q_{11}=\imath^{*}$ on $A$. This corresponds to a crossing which already has two boundary points connected by an arc as in case (3.) and which gets another arc glued to the remaining two boundary points:

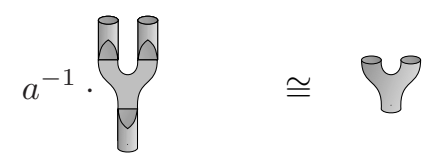

These relations imply that pre- and postcomposing $\widetilde{C}_{(\alpha, x)}$ with the appropriate $P_{k \ell}$ and $Q_{k \ell}$ indeed results in the maps of Remark 3.32 and thereby in the edges of the cube $Z\left(S_{T, \varepsilon}\right)$. 
The following corollary is now obvious and settles the question for the composition rule (1.11) raised in the introduction.

Corollary 4.5. Let $T$ be a plane diagram of an oriented $(p, q)$-tangle and $T^{\prime}$ be one of an oriented $(q, r)$-tangle such that the orientations match and the composition $T^{\prime} \circ T$ is well defined. Let $Z: \mathbf{2} \mathbf{C o b}^{\text {ext }} \rightarrow$ Vect $_{k}$ be a state sum open-closed TQFT that satisfies Bar-Natan's conditions. Then

$$
\llbracket T^{\prime} \circ T \rrbracket_{\varepsilon, Z} \cong \llbracket T^{\prime} \rrbracket_{\varepsilon, Z} \otimes_{A^{(q, \varepsilon)}} \llbracket T \rrbracket_{\varepsilon, Z}
$$

are isomorphic as complexes of $\left(A^{(r, \varepsilon)}, A^{(p, \varepsilon)}\right)$-bimodules, c.f. Remark 3.41

Remark 4.6. In order to generalize the composition of tangles to non-strongly separable algebras $A$, one could try the following. One sticks to the use of tensor products over $A$ for the composition, but one tries to avoid the use of anything that involves an $a^{-1}$. This excludes some of the $P_{j k}$ and $Q_{j k}$, but luckily not those $P_{1 k}$ and $Q_{1 k}$ that form the coequalizers. Nevertheless, apart from the $a^{-1}$, the open-closed cobordism depicted in (4.30) contains a window and will always yield a linear map with non-trivial kernel unless $A$ is strongly separable.

\subsection{Invariance under Reidemeister moves}

As we now have a construction of the tangle complex in local terms, i.e. from a composition of arcs and crossings, we are in a position to supply a proof of the invariance of its homology under Reidemeister moves in similarly local terms.

Theorem 4.7. Let $T, T^{\prime}$ be plane diagrams of oriented $(p, q)$-tangles, let $\varepsilon \in\{-1,+1\}$, and let $Z: \mathbf{2} \mathbf{C o b}^{\text {ext }} \rightarrow$ Vect $_{k}$ be an Euler-graded [Euler-filtered] state sum open-closed TQFT that satisfies Bar-Natan's conditions. If $T$ and $T^{\prime}$ are related by a plane isotopy or by a Reidemeister move, then the complexes $\llbracket T \rrbracket_{\varepsilon, Z}$ and $\llbracket T^{\prime} \rrbracket_{\varepsilon, Z}$ are homotopy equivalent as graded [filtered] complexes of $\left(A^{(q, \varepsilon)}, A^{(p, \varepsilon)}\right)$-bimodules.

The case of plane isotopies is already covered by Theorem 4.4. We now provide algebraic at the same time local proofs of the invariance up to homotopy equivalence under Reidemeister moves. These proofs are inspired by Bar-Natan's picture world, but it is nevertheless worthwhile spelling out the details in the language of knowledgeable Frobenius algebras. Thanks to the composition properties of state sum open-closed TQFTs described in the previous section, we need to check the Reidemeister moves

$$
R \text { mant } R+1
$$

only in the skein theoretic sense, meaning that any of the above diagrams can appear inside a possibly larger diagram, employing tensor products and compositions. Note that because our convention for orienting the tangle resolutions involves the checkerboard colouring $\varepsilon \in\{0,1\}$ of the tangle, we must take account for both possible colourings of the above diagrams.

We denote by $\left(A, C, \imath, \imath^{*}\right)$ the state sum knowledgeable Frobenius algebra associated with $Z$. In what follows we will omit the functor $Z$ around all diagrams of open-closed cobordisms.

\subsubsection{Reidemeister move one}

We show that the complex $\llbracket \Omega \rrbracket=(0 \longrightarrow \underline{A\{0\}} \longrightarrow 0)$ is homotopy equivalent to the complex

$$
\left[\left[{ }^{2} \Omega_{3}\right]\right]=\underline{(A \otimes C)\{2\}} \stackrel{d^{0}=\amalg}{\longrightarrow} A\{4\}
$$

where we have underlined the degree zero term of each complex. Note that neither of these complexes depends on the choice of checkerboard colouring $\varepsilon \in\{-1,+1\}$. The second complex 
always has $\left(n_{+}, n_{-}\right)=(1,0)$ regardless of the orientation of the tangle. Hence, we need only consider the one case.

Define the chain map $F: \llbracket \Omega \rrbracket \rightarrow \llbracket R \rrbracket$ as follows:

$$
F^{0}:=\mapsto \Omega(0), \quad F^{1}:=0 .
$$

The chain map property $d^{0} \circ F^{0}=0$ follows from the relations of the knowledgeable Frobenius algebra $\left(A, C, \imath, \imath^{*}\right)$. In fact, by Corollary 3.24 of [15] it is sufficient to compute the three topological invariants of the relevant composite open-closed cobordisms, and then Theorem 3.22 of [15] produces for us the sequence of relations that constitutes the proof. Next we define the chain map $G: \llbracket \Omega \rrbracket \rightarrow \llbracket \Omega \rrbracket$ by

$$
G^{0}:=\square^{\ominus}, \quad G^{1}:=0 .
$$

There are no conditions that need to be verified to see that this is a chain map.

The chain maps $F$ and $G$ define a homotopy equivalence between the complexes $\llbracket \Omega \rrbracket$ and 【R』. To see this, first note that $G \circ F=$ id by (1.5) since

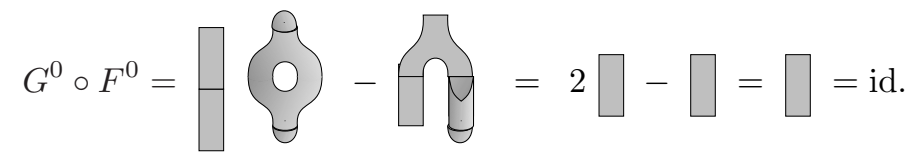

Define the chain homotopy $h$ : id $\rightarrow F \circ G$ whose only nonzero component is given by

$$
h^{1}:=\square_{0} .
$$

The equation id $-F^{0} \circ G^{0}=h^{1} \circ d^{0}$ is depicted as:

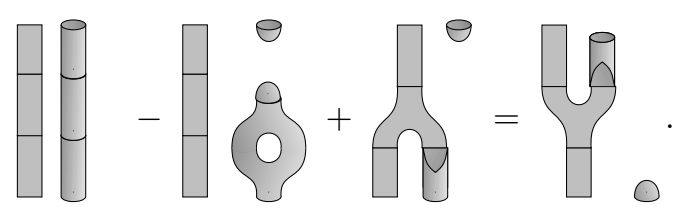

After applying appropriate relations and rearranging the terms, this equality follows from the relation 1.7]

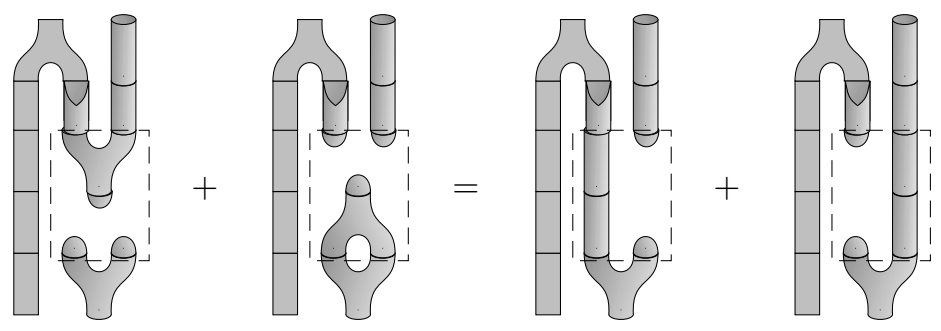

The equation id $-F^{1} \circ G^{1}=d^{0} \circ h^{1}$ follows from the knowledgeable Frobenius algebra axiom asserting that the map $\imath$ is an algebra homomorphism (preserves the unit) together with the unit axiom for the algebra $A$. Notice that all maps $F^{j}, G^{j}$, and $h^{j}$ are graded [filtered] of degree 0 if the appropriate grading shifts $\{-\}$ in (4.34) are taken into account. The other version of the first Reidemeister move can be treated similarly. 


\subsubsection{Reidemeister move two}

We show that the following complexes

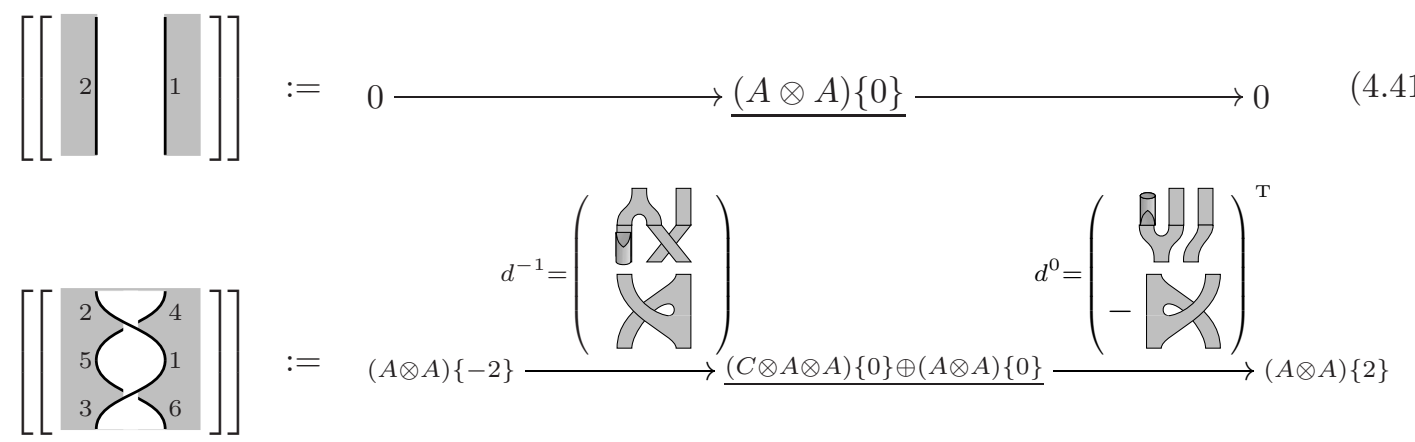

are homotopy equivalent. Note that in (4.42) we have $\left(n_{+}, n_{-}\right)=(1,1)$ regardless of the orientation of the tangle. We begin by defining chain maps $F:[[||]] \rightarrow\left[\left[\succ^{\prime}\right]\right]$ and $G:\left[\left[\succ^{\prime}\right]\right] \rightarrow[[||]]$ whose only nonzero components are given by the following diagram:

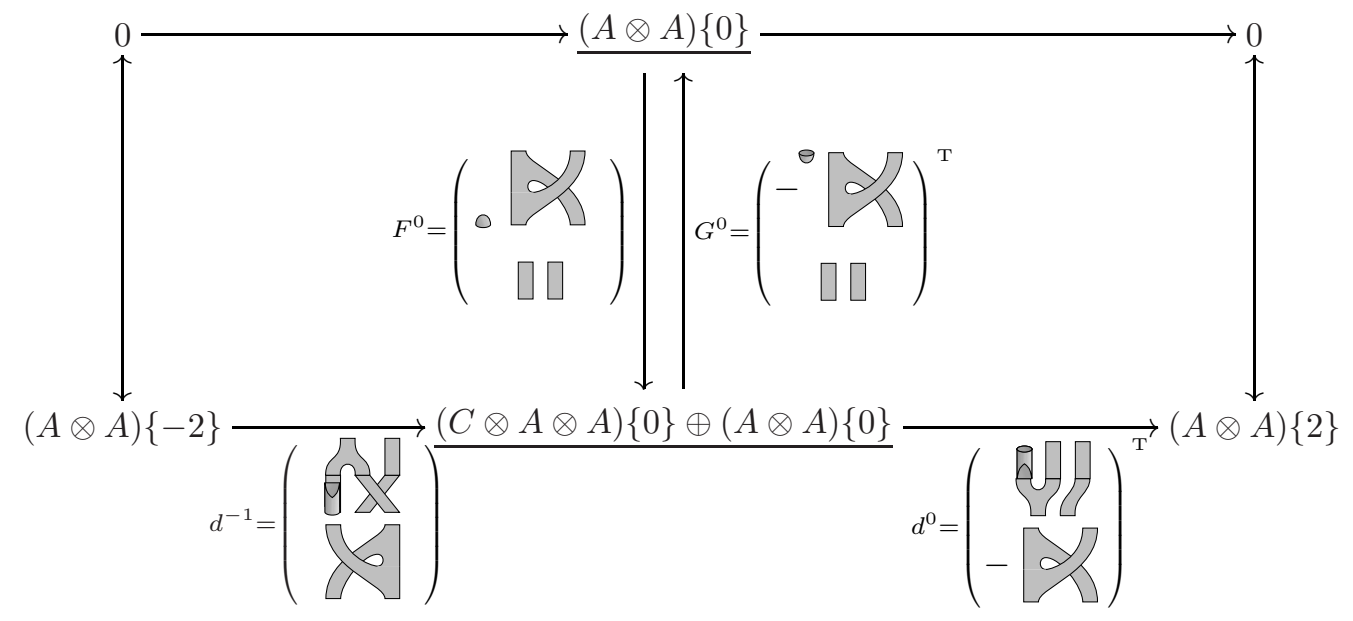

One can readily check that these maps define chain maps. The equation $d^{0} \circ F^{0}=0$ uses the fact that the zipper $\imath$ is an algebra homomorphism (preserves the unit) and the left unit axiom for the algebra $A$. The equation $G^{0} \circ d^{-1}=0$ follows from (3.54).

Note that $G \circ F=$ id by the relation (1.5) so the homotopy equivalence is established by defining a chain homotopy $h$ : id $\rightarrow F \circ G$. The nonzero components of this chain homotopy $h$ are given by

$$
h^{0}:=\left(\begin{array}{c}
\ominus \nabla Z \\
\lambda
\end{array}\right)^{\mathrm{T}} ; \quad h^{1}:=\left(\begin{array}{c}
\square \square \\
0
\end{array}\right) .
$$

The equation id $-F^{0} \circ G^{0}=h^{1} \circ d^{0}+d^{-1} \circ h^{0}$ is depicted below

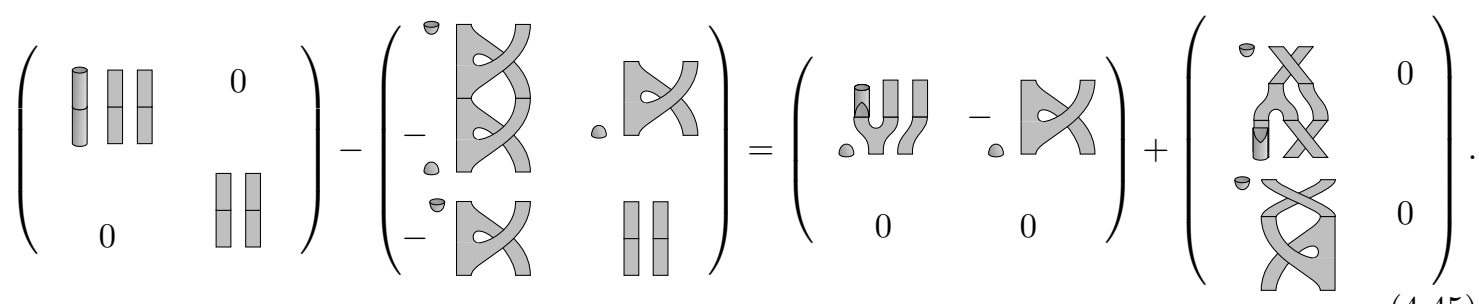


Only the first and third component of this matrix equation are non-trivial. The third component follows from (3.54). The first component can be written

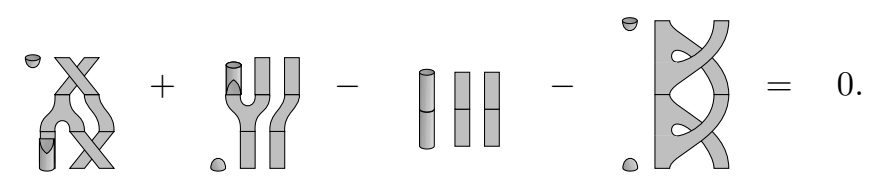

Noting that

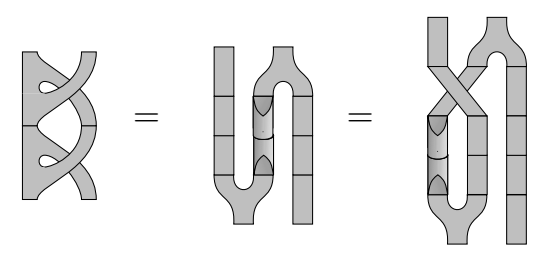

then (4.46) is just (1.7) applied to the cobordism

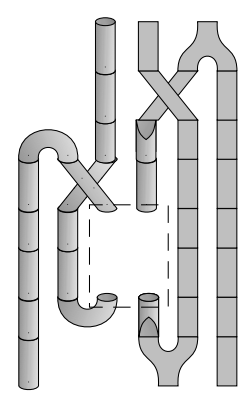

The equalities id $-F^{-1} \circ G^{-1}=h^{0} \circ d^{-1}$ and id $-F^{1} \circ G^{1}=h^{0} \circ d^{-1}+d^{0} \circ h^{1}$ follow from the identities

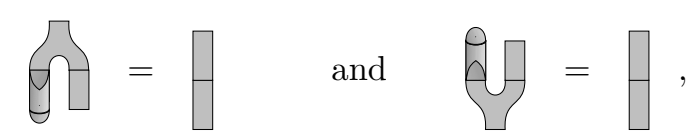

respectively.

For the opposite checkerboard colouring, $\varepsilon=-1$, of the complexes in (4.41) and (4.42) only the complex

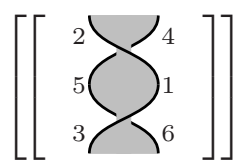

would change. In particular, one can verify that the complex becomes

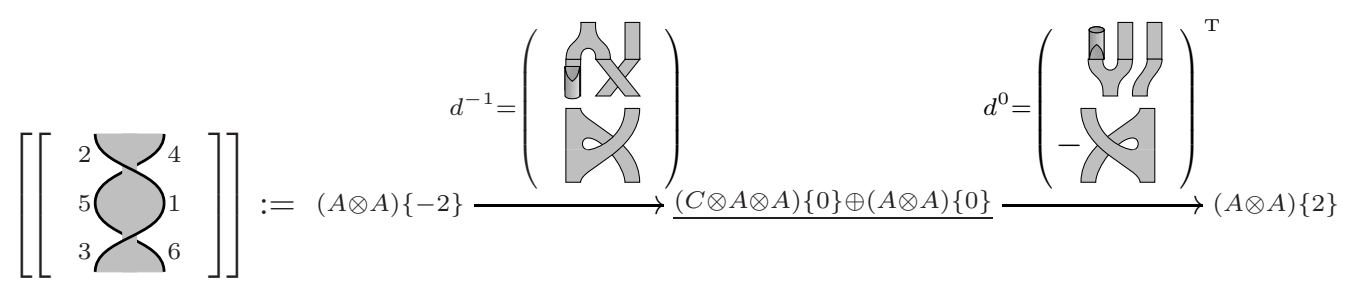

where only the saddle (3.48) has been replaced by (3.49) and vice versa. Performing a similar saddle switch for the chain maps $F$ and $G$ produces the required homotopy equivalence. Again, all maps $F^{j}, G^{j}$, and $h^{j}$ are graded [filtered] of degree 0 . Notice that the chain map $G$ : [[ $\left.\left.\zeta^{\prime}\right]\right] \rightarrow[[||]]$ is actually a strong deformation retract since $h \circ F=0$ by (1.5) and we have already shown that $G \circ F=$ id and id $-F \circ G=d \circ h+h \circ d$. Following the standard terminology we say that $F$ is the inclusion in a strong deformation retract. 


\subsubsection{Reidemeister move three}

To prove that the complex $\llbracket T \rrbracket$ is invariant up to homotopy equivalence under the third Reidemeister move, we require the following results from homological algebra.

Definition 4.8. Let $\Psi:\left(C_{0}^{r}, d_{0}\right) \rightarrow\left(C_{1}^{r}, d_{1}\right)$ be a morphism of complexes. The cone $\Gamma(\Psi)$ of $\Psi$ is the complex with terms $\Gamma^{r}(\Psi)=C_{0}^{r+1} \oplus C_{1}^{r}$ and the differentials $d^{r}=\left(\begin{array}{cc}-d_{0}^{r+1} & 0 \\ \Psi^{r+1} & d_{1}^{r}\end{array}\right)$.

If $\Psi$ is a morphism of graded [filtered] complexes, then the cone $\Gamma(\Psi)$ is a graded [filtered] complex.

Lemma 4.9. The cone construction is invariant up to homotopy equivalence under compositions with the inclusions in strong deformation retracts. That is, consider the diagram of morphisms and complexes on the right. If in that diagram $G^{0}$ is a strong deformation retract with inclusion $F_{0}$, then

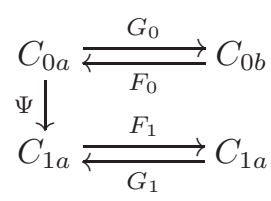
the cones $\Gamma(\Psi)$ and $\Gamma\left(\Psi \circ F_{0}\right)$ are homotopy equivalent, and if $G_{1}$ is a strong deformation retract with inclusion $F_{1}$, then the cones $\Gamma(\Psi)$ and $\Gamma\left(F_{1} \circ \Psi\right)$ are homotopy equivalent. Likewise, if $F_{0,1}$ are strong deformation retracts and $G_{0,1}$ the corresponding inclusions, the above statements remain true.

This lemma holds for complexes in any abelian category, i.e. if $\Psi$ and $F_{0}$ are morphisms of graded [filtered] complexes, then the cones $\Gamma(\Psi)$ and $\Gamma\left(\Psi \circ F_{0}\right)$ are homotopy equivalent as graded [filtered] complexes, etc..

We comment here that the proof of the above lemma is constructive, so that one can explicitly obtain the chain homotopies defining the above homotopy equivalence. In fact, Bar-Natan explicitly lists the chain maps and chain homotopy for Reidemeister move three which we encourage the reader to translate into the language of open-closed cobordisms.

For each $\xi \in \mathbb{Z}$ define the two chain maps $\mathcal{S}_{1}^{\xi}$ and $\mathcal{S}_{2}^{\xi}$ given by the diagram below:

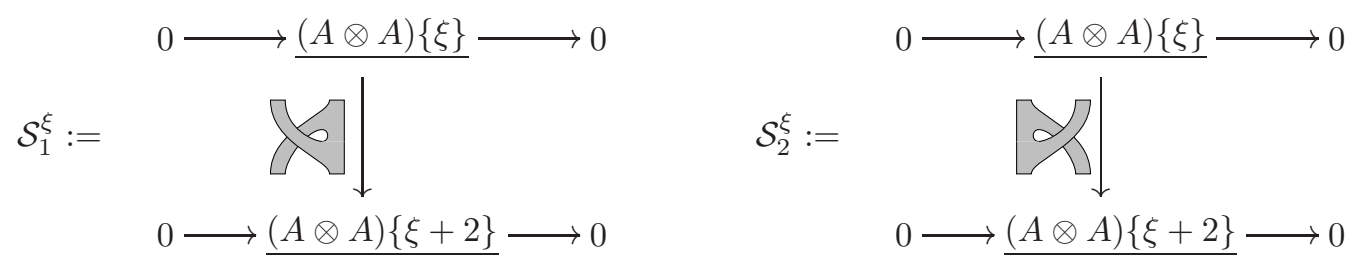

These are morphisms of graded [filtered] complexes.

Lemma 4.10. With $\mathcal{S}_{1}^{\xi}$ and $\mathcal{S}_{2}^{\xi}$ as in 4.51) we have that

$$
\begin{aligned}
& {\left[\left[\coprod_{3}^{1} \nearrow_{4}^{2}\right]\right]=\Gamma\left(\mathcal{S}_{1}^{(-4)}\right), \quad\left[\left[\coprod_{3}^{1} \nearrow_{4}^{2}\right]\right]=\Gamma\left(\mathcal{S}_{2}^{(-4)}\right),} \\
& {\left[\left[\coprod_{3}^{1}\right]\right]=\Gamma\left(\mathcal{S}_{1}^{(2)}\right)[1], \quad\left[\left[\sum_{3}^{1} \nearrow_{4}^{2}\right]\right]=\Gamma\left(\mathcal{S}_{1}^{(2)}\right)[1]}
\end{aligned}
$$

where $[s]$ is the cohomological degree shift.

By the discussion above the following complex

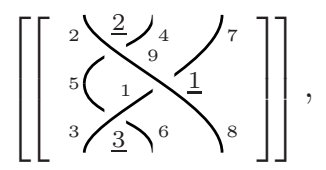


with the region to the right of the first strand shaded, is homotopy equivalent to the cone over the chain map

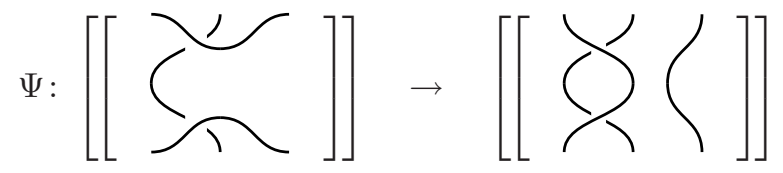

given by

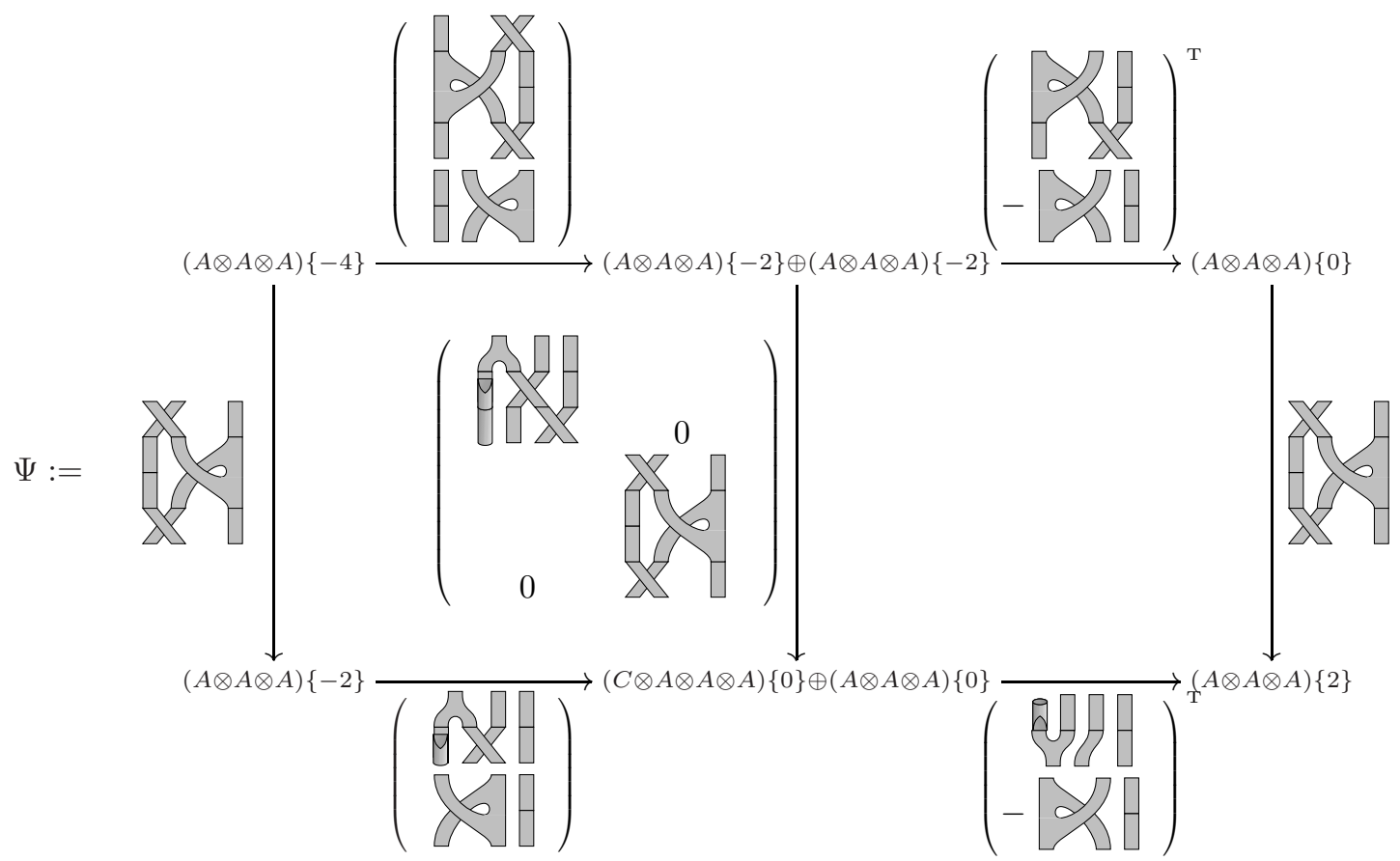

$(4.56)$

but by Lemma 4.9 the cone over the chain map $\Psi$ is homotopy equivalent to the cone over the composite of $\Psi$ with any strong deformation retract $G$. Taking $G$ as in (4.43) we have that [[ is homotopy equivalent to the cone of the map $\Psi^{\prime}=G \circ \Psi$ below

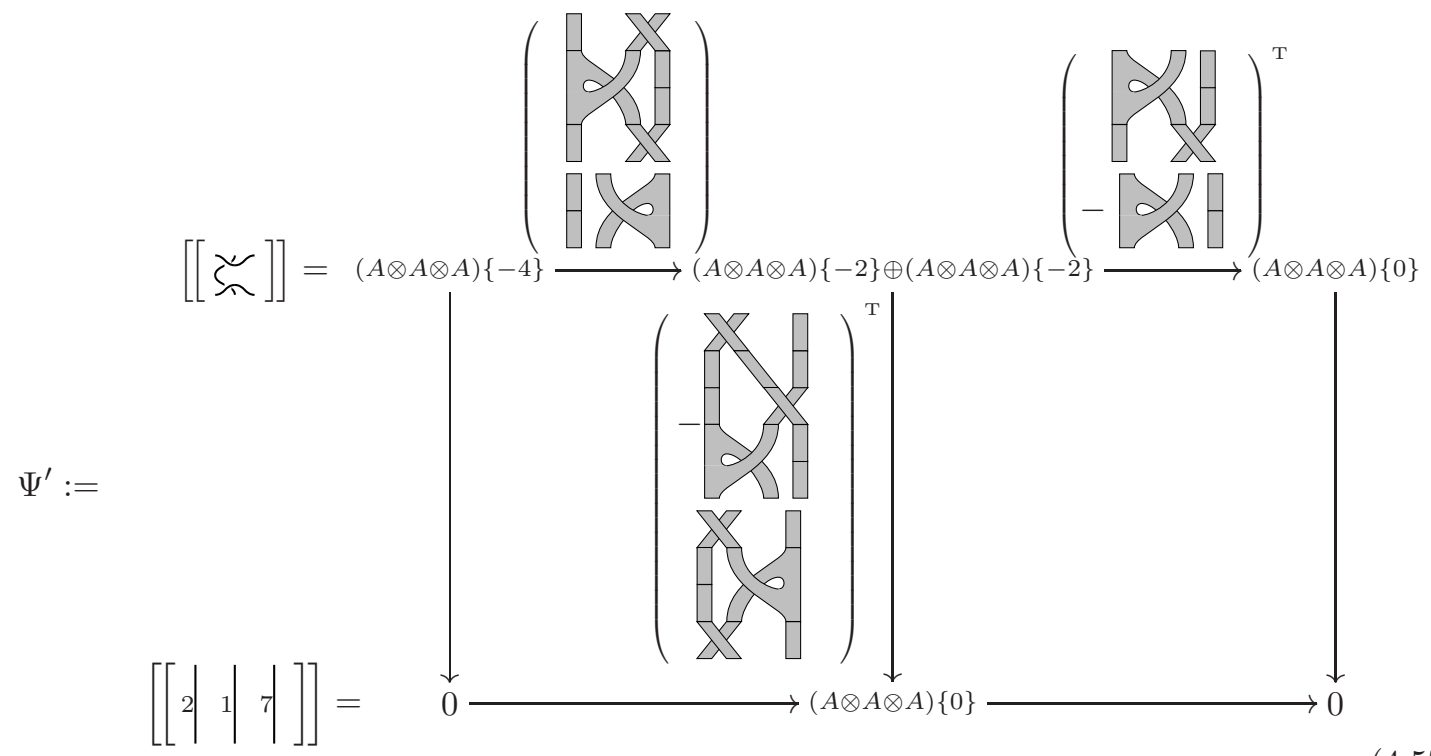

where we have left the enumeration on the bottom complex for clarity. Note that depending on the 
orientation of the tangle in (4.54) and on the number $n_{-}$of negative crossings, the complexes 4.56) and (4.57) can have either of their three terms in cohomological degree 0.

Similarly, by Lemma 4.10 the cone over the chain map

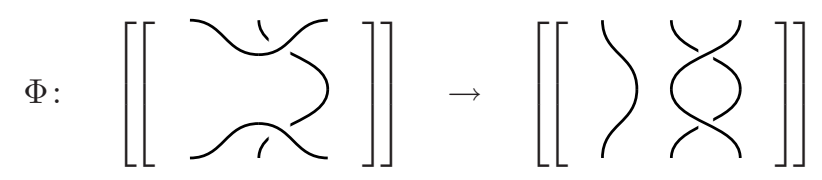

is homotopy equivalent to the complex

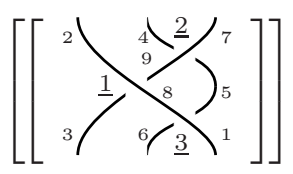

where $\Phi$ is given by

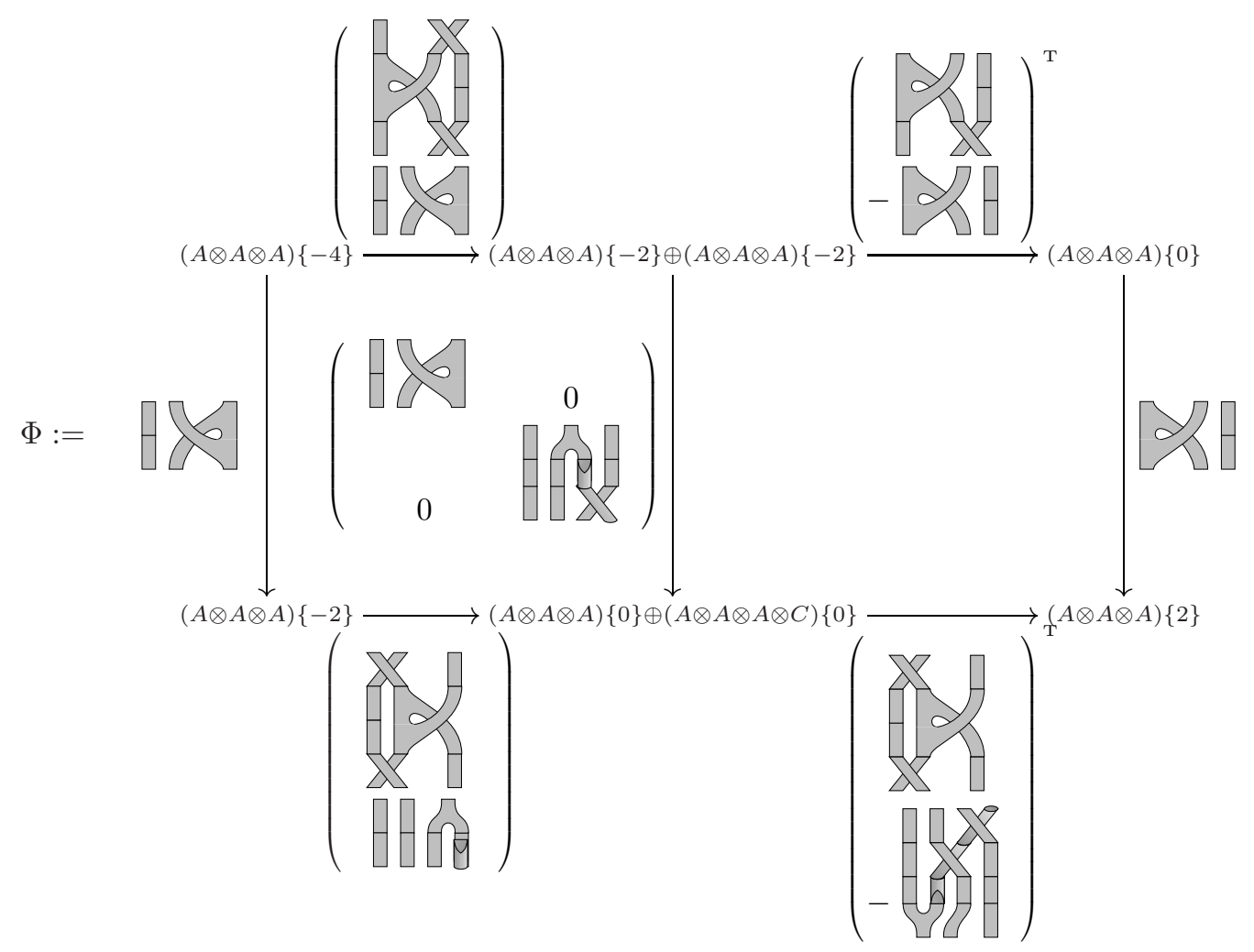

$(4.60)$

but again by Lemma 4.9 the cone $\Gamma(\Phi)$ is equal to the cone $\Gamma(G \circ \Phi)$ where $G$ is a strong deformation retract. We take $G$ as in (4.43), but with the arcs renumbered appropriately. This leads to a map $G$ whose only nonzero component $G^{0}$ is given by

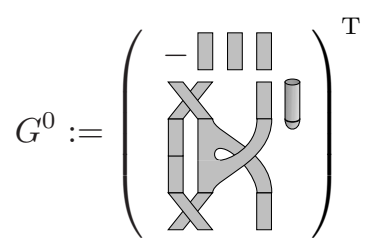

In order to make the enumeration of the complex $[[|| \mid]]$ the same as in (4.57), we also postcompose the composite $G \circ \Phi$ with the chain isomorphism $f$ of Proposition 3.33(2) whose only nonzero 
component is given as follows:

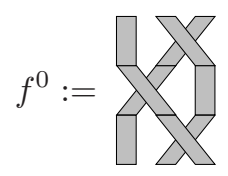

Thus, the complex (4.59) is homotopy equivalent to the cone of the composite $\Phi^{\prime}=f \circ G \circ \Phi$ given by

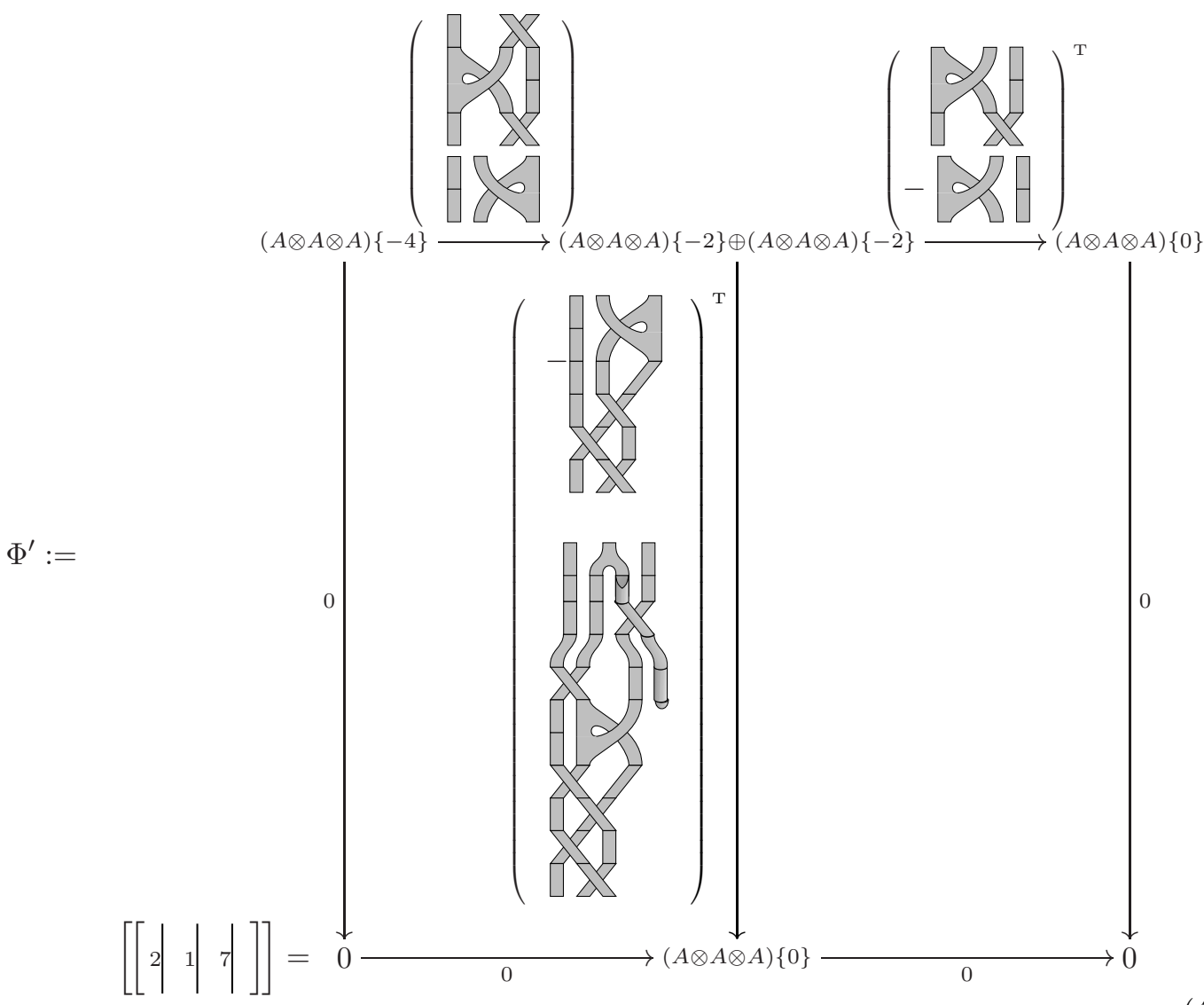

As an easy exercise in computing boundary permutations of open-closed cobordisms, one can see that the chain maps (4.57) and 4.63) are equal. Hence, their cones $\Gamma\left(\Psi^{\prime}\right)=\Gamma\left(\Phi^{\prime}\right)$ are equal, making the complexes

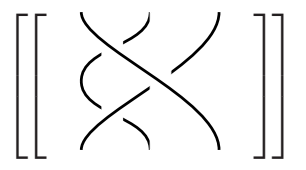

and

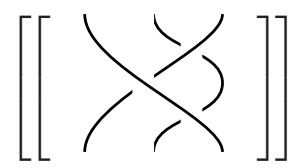

homotopy equivalent as graded [filtered] complexes by Lemma 4.9

We leave it to the reader to verify the other possible colouring of the Reidemeister three move as well as the Reidemeister three move for the other crossing configurations ${ }^{6}$.

\section{Acknowledgements}

We are grateful to Marco Mackaay, Simon Willerton, Christoph Schweigert, Mikhail Khovanov, Kevin Costello, Ivan Smith, and Paul Seidel for stimulating discussions. We are grateful to the European Union Superstring Theory Network for support. A.L. would like to thank the Max

\footnotetext{
${ }^{6}$ Bar-Natan's paper [5] provides the proof for the other version of Reidemeister three not proven here.
} 
Planck Institute in Potsdam, and both of us would like to thank the Mathematics Department at the University of Chicago for their hospitality.

The graphics were typeset using xypic 3.6 by Kristoffer Rose and Ross More; using pstricks 97 by Timothy van Zandt and others; and using dbnsymb and makefont by Dror Bar-Natan.

\section{References}

[1] M. G. Khovanov: A categorification of the Jones polynomial. Duke Math. J. 101, No. 3 (2000) 359-426, MR 1740682, math.QA/9908171

[2] D. Bar-Natan: On Khovanov's categorification of the Jones polynomial. Alg. Geom. Top. 2 (2002) 337-370, MR 1917056, math.QA/0201043.

[3] D. Bar-Natan: Khovanov homology for knots and links with up to 11 crossings. Available at http://www . math.toronto. edu/ drorbn/papers/KHTables/KHTables .pdf (2003).

[4] S. WehrLI: Khovanov homology and Conway mutation (2003). Preprint math.GT/0301312

[5] D. Bar-Natan: Khovanov's homology for tangles and cobordisms. Geom. Top. 9 (2005) 1443-1499, MR 2174270, math.GT/0410495

[6] L. Abrams: Two-dimensional topological quantum field theories and Frobenius algebras. J. Knot Th. Ramif. 5, No. 5 (1996) 569-587, MR 1414088

[7] S. F. SAwin: Direct sum decompositions and indecomposable TQFTs. J. Math. Phys. 36, No. 12 (1995) 6673-6680, MR 1359651, q-alg/9505026

[8] J. Kock: Frobenius algebras and 2D Topological Quantum Field Theories. London Mathematical Society Student Texts 59. Cambridge University Press, Cambridge, 2004. MR 2037238

[9] M. Jacobsson: An invariant of link cobordisms from Khovanov homology. Alg. Geom. Top. 4 (2004) 1211-1251, MR 2113903, math.GT/0206303

[10] M. Jacobsson: Khovanov's conjecture over $Z[c]$ (2003). Preprint math.GT/0308151

[11] M. G. Khovanov: Link homology and Frobenius extensions (2004). Preprint math.QA/0411447.

[12] E. S. LeE: An endomorphism of the Khovanov invariant. Adv. Math. 197, No. 2 (2005) 554-586, MR 2173845, math.GT/0210213.

[13] P. Turner: Calculating Bar-Natan's characteristic two Khovanov homology (2004). Preprint math.GT/0411225

[14] M. G. Khovanov: A functor-valued invariant of tangles. Alg. Geom. Top. 2 (2002) 665-741, MR 1928174, math.QA/0103190

[15] A. D. Lauda and H. Pfeiffer: Open-closed strings: Two-dimensional extended TQFTs and Frobenius algebras (2005). Preprint math.AT/0510664

[16] G. Moore and G. B. Segal: Lectures on Branes, $K$-theory and $R R$ Charges. Lecture notes from the Clay Institute School on Geometry and String Theory held at the Isaac Newton Institute, Cambridge, UK, available at http://www.physics.rutgers.edu/ gmoore/clay1/clay1.html and from the ITP Miniprogram 'The Duality Workshop' at Santa Barbara available at http://online.itp.ucsb.edu/online/mp01/moore1/ (2001-2002). 
[17] A. D. Lauda and H. Pfeiffer: State sum construction of two-dimensional open-closed Topological Quantum Field Theories (2006). Preprint math.QA/0602047

[18] J. Rasmussen: Khovanov homology and the slice genus (2004). Preprint math.GT/0402131

[19] S. MacLane: Categories for the working mathematician. Graduate Texts in Mathematics 5. Springer, Berlin, 1998. MR 1712872

[20] J. McCleary: A user's guide to spectral sequences. Cambridge Studies in Advanced Mathematics 58. Cambridge University Press, Cambridge, 2001. MR 1793722

[21] M. MackaAy, P. Turner and P. Vaz: A remark on Rasmussen's invariant of knots (2006). Preprint math.GT/0509692

[22] J. Rasmussen: Knot polynomials and knot homologies. Fields Inst. Comm. 47 (2005) 261-280, MR 2189938 math.GT/0504045 